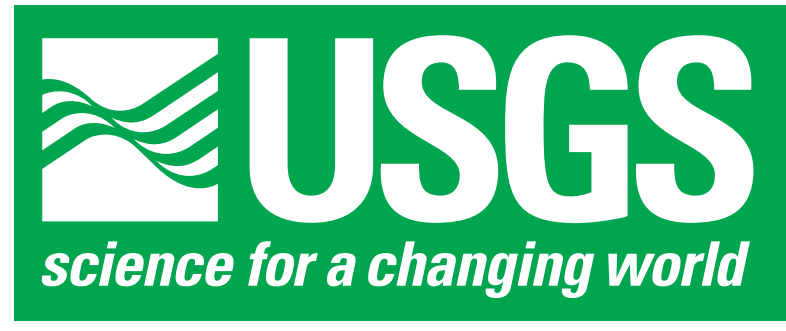

\title{
Method for Estimating Pesticide Use for County Areas of the Conterminous United States
}

U.S. Geological Survey

Open-File Report 00-250

U.S. DEPARTMENT OF THE INTERIOR

U.S. GEOLOGICAL SURVEY 


\section{Method for Estimating Pesticide Use for County Areas of the Conterminous United States}

Gail P. Thelin ${ }^{1}$ and Leonard P. Gianessi ${ }^{2}$

\section{U.S. GEOLOGICAL SURVEY}

Open-File Report 00-250

0
1
0
0
$\infty$

${ }^{1}$ U.S. Geological Survey

${ }^{2}$ National Center for Food and Agricultural Policy 


\section{U.S. DEPARTMENT OF THE INTERIOR \\ BRUCE BABBITT, Secretary}

U.S. GEOLOGICAL SURVEY

Charles G. Groat, Director

The use of firm, trade, and brand names in this report is for identification purposes only and does not constitute endorsement by the U.S. Geological Survey.

For additional information write to:

Chief of Pesticide National Synthesis U.S. Geological Survey

Placer Hall, Suite 2012

6000 J Street

Sacramento, CA 95819-6129
Copies of this report can be purchased from:

U.S. Geological Survey Information Services

Box 25286

Federal Center

Denver, CO 80225 


\section{CONTENTS}

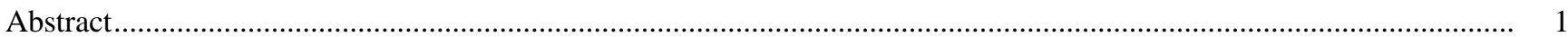

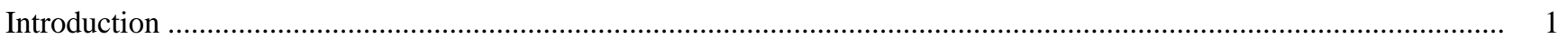

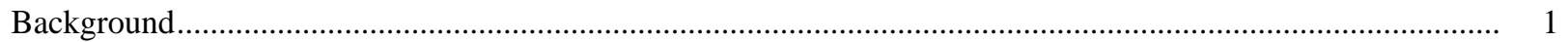

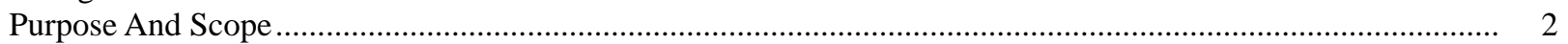

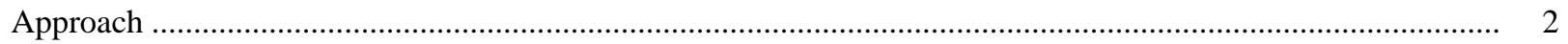

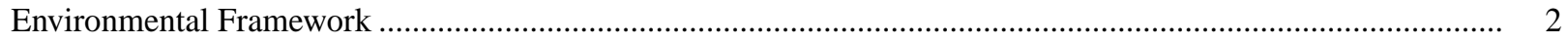

The National Center for Food and Agricultural Policy's State Pesticide Use Database.................................................... 4

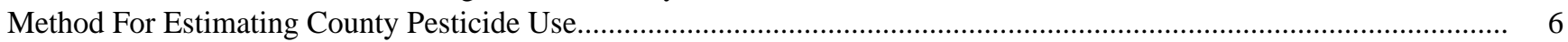

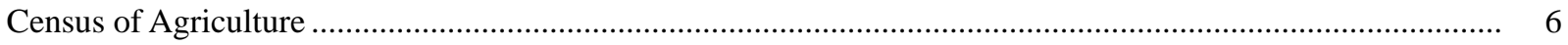

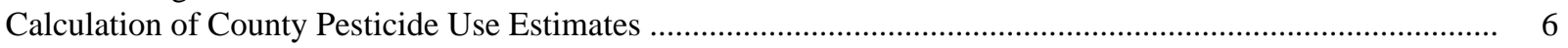

Limitations of the County Pesticide Use Data for Water-Quality Assessment............................................................. 7

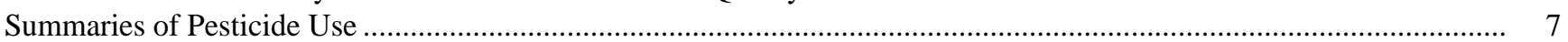

The National Water-Quality Assessment's Study Unit Summaries ........................................................................... 8

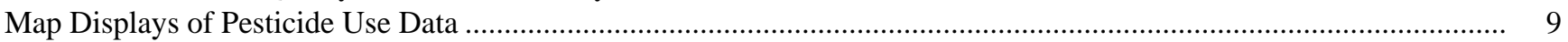

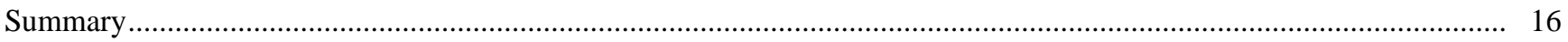

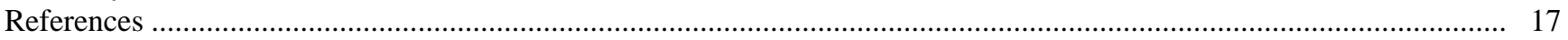

Figure

1. Locations of National Water-Quality Assessment Program study units for which pesticide use estimates were developed.

2. Seven records associated with Steele County, Minnesota, which display the area of the county associated with

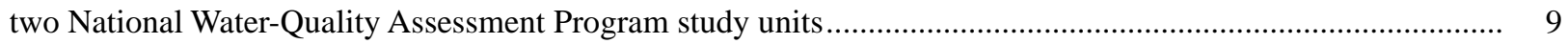

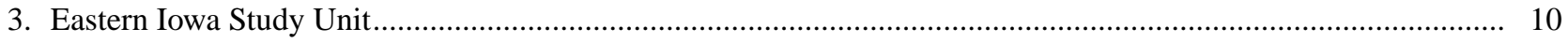

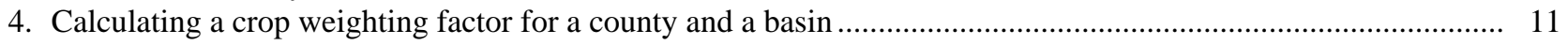

5. Average annual amount of active ingredient of acetochlor $(\boldsymbol{A})$ and metolachlor $(\boldsymbol{B})$ applied to cropland

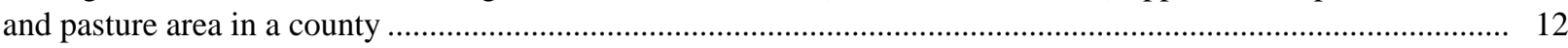

6. Average annual amount of active ingredient of methyl bromide $(\boldsymbol{A})$ and chlorpyrifos $(\boldsymbol{B})$ applied to

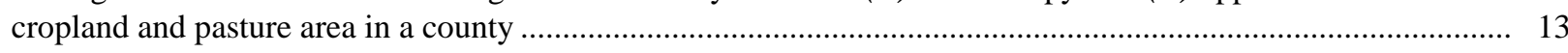

7. Average annual atrazine use applied to cropland categories mapped from Advanced Very High Resolution Radiometer data

Tables

1. Pesticide codes for compound names contained in the National Center for Food and Agricultural Policy's 1992-1995 state database.

2. Crop codes and crop names with corresponding Census of Agriculture data codes....................................... 18

3. Summary of national pesticide use, 1992-1995, derived from county pesticide use information sorted by total application in pounds active ingredient in descending order.

4. Primary crops that account for use of each pesticide, 1992-1995 ................................................................. 24

5. Ranking of pesticides used in National Water-Quality Assessment Program study units during 1992-1995 ........ 39 
Abbreviations and Acronyms

AVHRR
CALEPA
EIWA
FIPS
GIS
NAPIAP
NASS
NCFAP
NAWQA
STCROP
STPESTCROP

USDA

USGS
Advanced Very High Resolution Radiometer

California Environmental Protection Agency

Eastern Iowa Basins

Federal Information Processing Standard

Geographic Information System

National Agricultural Pesticide Impact Assessment Program

National Agricultural and Statistics Service

National Center for Food and Agricultural Policy

National Water-Quality Assessment (Program)

State Crop Code

A key that includes the two-digit state FIPS code, four-digit pesticide code and three-digit crop code

U.S. Department of Agricultural

U.S. Geological Survey 


\section{Method for Estimating Pesticide Use for County Areas of the Conterminous United States}

\author{
By Gail P. Thelin and Leonard P. Gianessi
}

\section{ABSTRACT}

Information on the amount and distribution of pesticide compounds used throughout the United States is essential to evaluate the relation between water quality and pesticide use. This information is the basis of the U.S. Geological Survey's National Water-Quality Assessment (NAWQA) Program studies of the effects of pesticides on water quality in 57 major hydrologic systems, or study units, located throughout the conterminous United States. To support these studies, a method was devised to estimate county pesticide use for the conterminous United States by combining (1) state-level information on pesticide use rates available from the National Center for Food and Agricultural Policy, and (2) countylevel information on harvested crop acreage from the Census of Agriculture. The average annual pesticide use, the total amount of pesticides applied (in pounds), and the corresponding area treated (in acres) were compiled for the 208 pesticide compounds that are applied to crops in the conterminous United States. Pesticide use was ranked by compound and crop on the basis of the amount of each compound applied to 86 selected crops. Tabular summaries of pesticide use for NAWQA study units and for the Nation were prepared, along with maps that show the distribution of selected pesticides to agricultural land.

\section{INTRODUCTION}

\section{Background}

More than 400,000 tons of pesticides are applied annually to agricultural land in the United States.
Information on agricultural pesticide use is available from several national and state pesticide use surveys and reports, along with a variety of research reports on specific crops or states. The type and detail of data that are reported varies widely between federal and state programs, making it difficult to rely on a single source for complete information. Three examples of such programs are the U.S. Department of Agriculture's (USDA) National Agricultural and Statistics Service (NASS), the Census of Agriculture, and the state of California's Department of Pesticide Regulation. The NASS provides state summaries of pesticide use annually for major field crops, which include corn, wheat, soybeans, cotton, potatoes, and, in selected years, peanuts, rice, sorghum, and tobacco. NASS conducts a second survey that targets specialty crops, alternating yearto-year between vegetables (even-numbered years) and fruit and nut crops (odd-numbered years). The NASS data are based on a statistical sample of farms in states that account for at least 80 percent of United States production for a specific crop. Summaries of the average application rates of major pesticide ingredients and the percentage of crop area (in acres) treated are published annually by NASS. In comparison, the United States Census of Agriculture conducts a survey every 5 years of all farms within the United States that have an annual farm income of $\$ 1,000$ or more. This enumeration includes information on the type, quantity, and cost of agricultural chemicals used on each farm. The data, reported by county, include the number of farms and the number of acres on which broad classes of chemicals are used (for example, herbicides and insecticides). In contrast, the California Environmental Protection Agency's (CALEPA) Department of Pesticide Regulation collects state-wide information annually, by location, on the amount of individual pesticides used on all crops-major field crops, specialty crops, orchards, and vineyards.

Each of these surveys and reports provides useful information; however, these sources do not provide all of the information needed to conduct a national 
assessment of pesticide use. NAWQA requires data on the full range of pesticide compounds used on major crops (such as corn, soybeans, wheat, and other grains) and specialty crops (such as vegetables, orchards, and vineyards) throughout all the states. The level of detail reported for the state of California by the Department of Pesticide Regulation is considered optimal, but not widely available. Other states, such as New York, Texas, Arizona, and Oregon, are considering or have adopted similar programs. Until these data are uniformly available for all states, however, an alternative source and method of estimating pesticide use are needed.

\section{Purpose and Scope}

This report describes the method used to develop county estimates of agricultural pesticide use and the application of these data for estimating pesticide use in the NAWQA study units. The Cook Inlet (Alaska) and Oahu (Hawaii) study units were not included in the analyses. State pesticide use-coefficients developed for 208 active ingredients, or pesticides, and 86 crops (Gianessi and Anderson, 1995) were applied to Census of Agriculture county crop acreage to estimate average annual pesticide usage totals for counties, including the amount of pesticides applied (in pounds) and the corresponding area treated (in acres). County pesticide data were used to profile pesticide use for the NAWQA study units, to rank use among the study units, and to compare results with national use. Maps for selected pesticides were prepared by combining county pesticide use data with the location of agricultural land use.

\section{Approach}

The purpose of the U.S. Geological Survey's (USGS) National Water-Quality Assessment (NAWQA) Program, which began in 1991, is to assess the status and trends of the quality of ground and surface waters in 59 major hydrologic systems ( 57 in the conterminous United States) distributed throughout the United States. These hydrologic systems, referred to as "study units" (fig. 1), represent the diverse hydrologic conditions found in the United States, encompass approximately one-half of its land area, and contain about 60 percent of its water resources. More than half of the Nation's population and total water use is contained within these study units. The study units also encompass the rich diversity of agriculture that is found across the Nation. Major crops, such as wheat and other grains, corn, and soybeans, dominate the study units, whereas a variety of specialty crops, such as vegetables, cotton, tobacco, sorghum, and rice, are also well represented (Gilliom and Thelin, 1997). In all, about one-half of the Nation's harvested cropland and pastureland, and approximately two-thirds of the national use of pesticides used for agriculture, occur in these study units.

The potential effect of pesticides on water quality is an issue of common concern throughout the nation, and the NAWQA Program addresses this issue in its assessment of the effects of pesticides in each study unit and nationally. Pesticides in both surface water and ground water are systematically sampled in each study unit, followed by analysis and interpretation of findings using commonly prescribed analytical and interpretative methods. The study units' results are combined and analyzed at regional and national scales to assess the effects of pesticide use on the quality of the nation's water resources.

Information on the amount and distribution of pesticide compounds used in the United States is an essential element in establishing relations between pesticide use and water quality. The NAWQA studies use this information to evaluate pesticide loads and concentrations in surface and ground waters, and to explain the variability of water-quality conditions among different agricultural settings and among surface-water basins of varying sizes. With an understanding of the relations between agricultural management practices (such as timing and amount of pesticide application, and cropping patterns), physical characteristics of the site, and water quality, the study-unit findings can be extrapolated to areas of the country outside of study unit areas that have not been sampled but that have similar characteristics. Lastly, and perhaps most importantly, these data provide information for prioritizing areas that should be monitored for pesticide use.

\section{Environmental Framew ork}

Information on the natural features and human influences that affect water quality form an environmental framework, which is used for NAWQA design and data analysis. Tabular databases and digital maps, stored in a Geographic Information System (GIS), provide geographically referenced information on geology, soils, streams, and several of the features that 


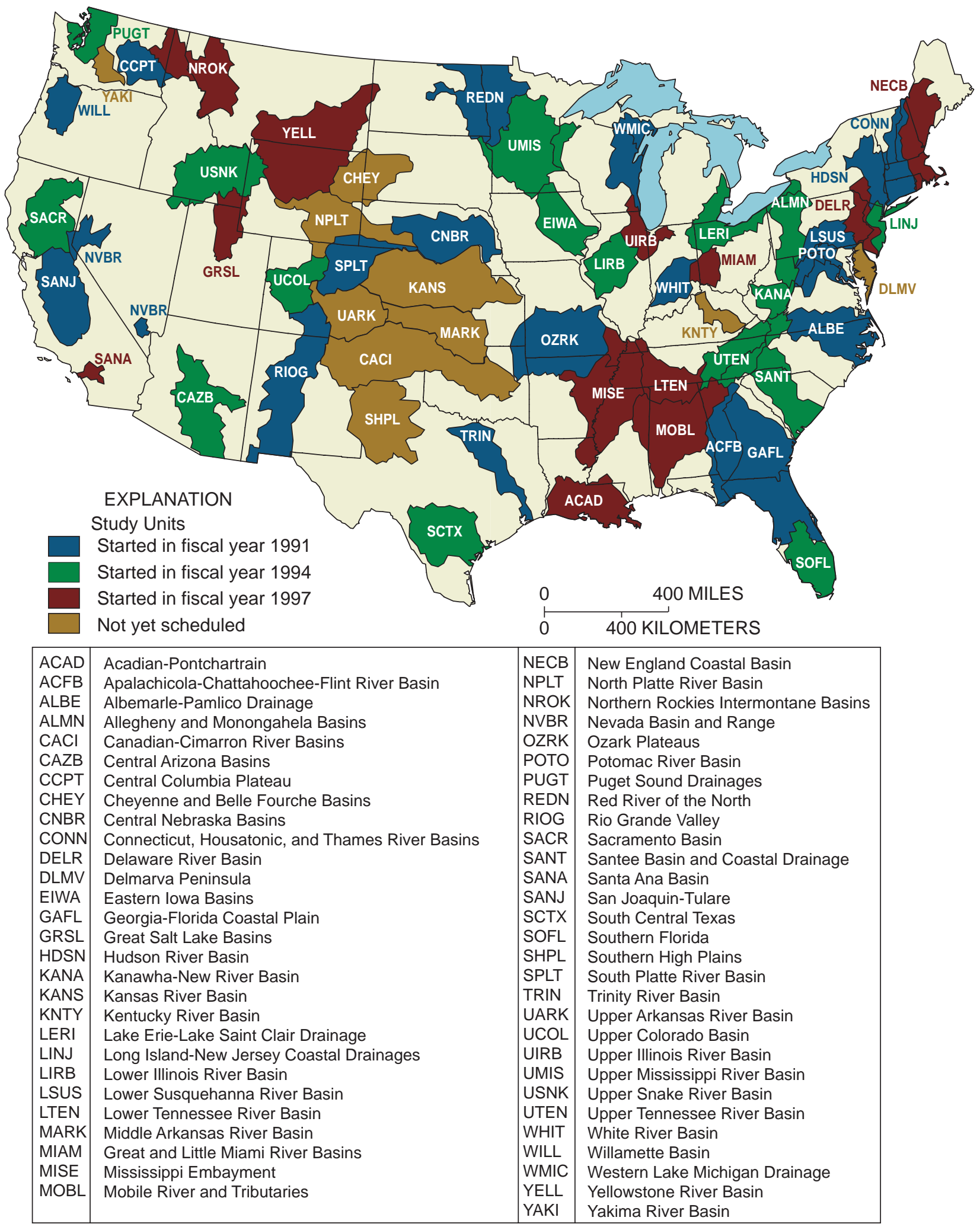

Figure 1. Locations of National W ater-Quality Assessment Program study units for which pesticide use estimates were developed. 
characterize the man-made landscape, such as land use. Data that describe these environmental characteristics are collected from a variety of sources, and consequently, range in spatial scale, coverage, and date of compilation. It is important, however, that each source of information is consistent in spatial scale and amount of detail so that comparisons and interpretations can be made. The NAWQA environmental framework for national analysis is based on many small-scale databases covering information on various geographical features that are available on the entire United States.

Data that describe the environmental setting of each ground-water and surface-water site sampled by NAWQA are assembled and used to explore and understand the relations between these natural and anthropogenic factors and water quality. The framework also serves as a common background to compare water quality in different parts of the country. For example, once relations between water quality and pesticide applications to irrigated corn grown on sandy soils in a particular area within a study unit are determined, then these relations can be directly compared with other areas of the country that share similar physical characteristics and crop management practices. The framework may be used to interpret and understand why the occurrence and concentrations of pesticides differ for various environmental settings.

Land use is one of the most important and unifying elements of the environmental framework because it serves as a surrogate for many of the human-related activities that affect water quality. The location and amount of different land uses serve as a backdrop that other characteristics of land use can be linked to, such as agricultural management practices, including irrigation, crop rotation, tillage, and pesticide use. In addition, other information related to land use could be used to update and refine these mapped data. For example, U.S. Census Bureau population density data have been used to update residential use patterns and to characterize residential use by population density category (Hitt, 1994). Census of Agriculture data (U.S. Department of Commerce, 1995) also have been used to characterize agricultural land use according to a crop group classification scheme (Gilliom and Thelin, 1997).

Estimates of the amounts and spatial distributions of agricultural and nonagricultural pesticide use are important elements in the NAWQA environmental framework. Agricultural uses of pesticides include application to crops, pretreatment of seeds, and treat- ment of livestock with insecticides, whereas nonagricultural uses vary from applications to rangeland, forestland, highway rights-of-way, turf, and a variety of home uses. Few data on nonagricultural total use of pesticides are available at this time, whereas data on agricultural uses have been collected for many years. Until more comprehensive data on nonagricultural pesticide use are available, the NAWQA environmental framework includes only agricultural pesticide-use estimates.

\section{THE NATIONAL CENTER FOR FOOD AND ARICULTURAL POLICY'S STATE PESTICIDE USE DATABASE}

The National Center for Food and Agricultural Policy (NCFAP) is a nonprofit research organization that has reviewed and incorporated information from more than 130 national and state surveys and reports into a comprehensive national agricultural pesticide use database. The database was developed to support NCFAP's research, policy analysis, and outreach programs that focus on issues related to agriculture. One component of NCFAP research and policy analysis addresses concerns about the benefits and risks associated with chemicals used in food production and processing.

The NCFAP database contains estimates of pesticide use by crop for each of the 48 conterminous states. Pesticide use is reported as the total amount of a pesticide applied and the area treated with the pesticide for each state and crop, and compound combination. Pesticide use-coefficients, which are required to calculate pesticide use, are also available from the database. The coefficients include the average amount of an active ingredient applied annually per crop acre and the percentage of crop area treated. Tables 1 and 2 respectively list the 208 compounds used in agriculture and the 86 crops for which NCFAP has developed pesticide use-coefficients.

The primary data sources used by NCFAP to develop a state pesticide use report included data compiled by three USDA programs and one state source: the NASS, the National Agricultural Pesticide Impact Assessment Program (NAPIAP), the Cooperative Extension Service, and the CALEPA. In some instances, data for a specific crop or state were not available. For these instances, the state pesticide use- 
Table 1. Pesticide codes for compound names contained in the National Center for Food and Agricultural Policy's $1992-1995$ state database

\begin{tabular}{|c|c|c|c|c|c|c|c|}
\hline $\begin{array}{c}\text { Pesticide } \\
\text { code }\end{array}$ & Compound & $\begin{array}{c}\text { Pesticide } \\
\text { code }\end{array}$ & Compound & $\begin{array}{c}\text { Pesticide } \\
\text { code }\end{array}$ & Compound & $\begin{array}{c}\text { Pesticide } \\
\text { code }\end{array}$ & Compound \\
\hline 8008 & 1,3-D & 5014 & Captan & 6014 & Diazinon & 6022 & Ethion \\
\hline 1302 & 2,4-D & 6006 & Carbaryl & 1298 & Dicamba & 9012 & Ethofumesate \\
\hline 1308 & 2,4-Db & 6007 & Carbofuran & 1865 & Dichlobenil & 6023 & Ethoprop \\
\hline 6001 & Abamectin & 5026 & Carboxin & 1005 & Diclofop & 6024 & Ethyl parathion \\
\hline 6002 & Acephate & 1299 & Chloramben & 6016 & Dicofol & 5051 & Etridiazole \\
\hline 3000 & Acetochlor & 4008 & Chlorimuron & 6082 & Dicrotophos & 6025 & Fenamiphos \\
\hline 1002 & Acifluorfen & 8000 & Chloropicrin & 9015 & Diethatyl ethyl & 5032 & Fenarimol \\
\hline 1863 & Alachlor & 5007 & Chlorothalonil & 1374 & Difenzoquat & 6026 & Fenbutatin oxide \\
\hline 6003 & Aldicarb & 6009 & Chlorpyrifos & 6064 & Diflubenzuron & 9003 & Fenoxaprop \\
\hline 1982 & Ametryn & 1913 & Chlorsulfuron & 3001 & Dimethenamid & 7203 & Fenpropathrin \\
\hline 6091 & Amitraz & 7010 & Clethodim & 7004 & Dimethipin & 6070 & Fenvalerate \\
\hline 5018 & Anilazine & 7204 & Clofentezine & 6017 & Dimethoate & 5017 & Ferbam \\
\hline 9048 & Asulam & 9001 & Clomazone & 3006 & Dimethomorph & 9007 & Fluazifop \\
\hline 1980 & Atrazine & 4002 & Clopyralid & 5035 & Dinocap & 3003 & Flumetsulam \\
\hline 6004 & Azinphos-methyl & 5011 & Copper & 1366 & Diphenamid & 1998 & Fluometuron \\
\hline 1362 & Benefin & 6010 & Cryolite & 1950 & Diquat & 4010 & Fomesafen \\
\hline 5001 & Benomyl & 1369 & Cyanazine & 6018 & Disulfoton & 6028 & Fonofos \\
\hline 7009 & Bensulfuron & 2069 & Cycloate & 1991 & Diuron & 6071 & Formetanate $\mathrm{HCl}$ \\
\hline 1098 & Bensulide & 6081 & Cyfluthrin & 5033 & Dodine & 5031 & Fosetyl-Al \\
\hline 1287 & Bentazon & 3008 & Cymoxanil & 4001 & DSMA & 8013 & Gibberellic acid \\
\hline 6063 & Bifenthrin & 6011 & Cypermethrin & 6019 & Endosulfan & 1099 & Glyphosate \\
\hline 1809 & Bromacil & 6012 & Cyromazine & 1948 & Endothall & 2070 & Hexazinone \\
\hline 1116 & Bromoxynil & 8015 & Cytokinins & 1414 & EPTC & 7001 & Imazamethabenz \\
\hline 6005 & $\mathrm{Bt}$ & 5008 & DCNA & 6020 & Esfenvalerate & 4005 & Imazaquin \\
\hline 1839 & Butylate & 1872 & DCPA & 9009 & Ethalfluralin & 9000 & Imazethapyr \\
\hline 8017 & Cacodylic acid & 9014 & Desmedipham & 7003 & Ethephon & 3004 & Imidacloprid \\
\hline
\end{tabular}

coefficients were generated on the basis of opinions of USDA Extension service specialists who were experienced with state cropping and management practices. In a few cases, there were no published surveys or information from Extension service specialists on a specific crop or compound; in these cases, similar pesticide use profiles of a neighboring state were used in place of the survey.

The coefficients represent the average annual application rates to cropland only, and do not include the commercial treatment of seeds, use in greenhouses or on ornamentals, or postharvest treatment of crops. The use-coefficients are based on the statewide average use for a compound and crop, and therefore do not reflect local variations in use as a result of local environmental variables (such as soil and climate) and crop management practices. The pesticide use-coefficients do not represent a specific year, but rather an integra- tion of data collected over a 4-year period because NCFAP developed the reported information from multiple sources collected from 1992 to 1995.

Although the NCFAP report was compiled from multiple sources that represent four different years, it has been verified by review and comparison with other published data. In addition, over 30 chemical manufacturing companies have reviewed the state and crop pesticide use estimates contained in the report. Through this informal process, NCFAP was able to resolve most discrepancies between published survey results and comments on use and rates of use from the reviewers.

The NCFAP report provides estimates of state and national pesticide use. NAWQA studies, however, require estimates of pesticide use for study units and smaller areas within study units. The following section briefly describes how NCFAP state pesticide use-coef- 
ficients were combined with county crop acreage to develop a county database of pesticide use.

\section{METHOD FOR ESTIM ATING COUNTY PESTICIDE USE}

\section{Census of Agriculture}

County crop acreage data from the 1992 Census of Agriculture (hereinafter referred to as "Census") were used to determine the amount of different pesticides used on different crops in each county. The Census is the only nationally consistent, complete source of crop acreage data. It is the leading source of statistics on agriculture in the United States and is collected every 5 years by mail surveys for all places from which $\$ 1,000$ or more of agricultural products were produced and sold or normally would have been sold during the census year (U.S. Department of Commerce, 1995). Planted and harvested crop acreage is reported for those crops that have pesticide use-coefficients that were developed by NCFAP. For purposes of this report, county crop acreage always is "harvested" acreage from the Census.

Census information is available as a computerreadable file with individual items referenced by a numeric code. Crop acreage was retrieved from the Census using the numeric crop code for crops listed in table 2 . There were 3,069 counties in the conterminous United States that reported crop acreage during the 1992 Census. Data files were created for each crop that had pesticide use-coefficients developed by NCFAP. Each crop file contained a two-digit state FIPS (Federal Information Processing Standard) code and a threedigit county code and crop acreage (for example, 06019 comprises " 06 " for the state of California and "019" for Fresno County). In some instances, the Census reports several categories of crops such as sorghum, corn, and sugar cane. For example, the crop sorghum is classified into three categories: sorghum for grain, sorghum for dry forage, and sorghum for silage (table 2). In these cases, the various categories of a crop were summed to produce total acreage for a county. The county crop acreage files were appended. The crop code developed by NCFAP (table 2) was added and combined with the two-digit state FIPS code to produce a state crop code (STCROP) that could be used to link crop acreage to the NCFAP state pesticide usecoefficients.

\section{Calculation of County Pesticide Use Estimates}

The NCFAP state pesticide use data file had seven columns of information: state name, compound name, pesticide code, crop name, crop code, average amount (in pounds) of an active ingredient applied annually, and percentage of area (in acres) treated with the pesticide active ingredient. The data file contained 16,000 records - one record for each pesticide and crop combination for each state. The two-digit state FIPS code corresponding to the state name was added to the file to create the same STCROP code that was contained in the crop acreage file. The STCROP code is used to temporarily link the two files and to retrieve county crop acreage for compounds that have a pesticide use coefficient. For example, in Texas (state FIPS code 48), the state pesticide use data file contained pesticide use-coefficients for 21 compounds that are used with rice (crop code 605). Using the STCROP code 48605, the harvested crop acreage file was checked for any data that were reported for this code. For Texas, 17 counties reported rice acreage in 1992. The crop acreage for rice in the 17 counties was retrieved and written to a new file 21 times - each time for a different compound; that is, once for propanil, once for triclopyr, and so on. The file now contained 357 records that included 17 records (counties) written for each of the $21 \mathrm{com}$ pounds that are applied to rice.

This procedure was repeated for each STCROP code that had a pesticide use-coefficient in the state pesticide use file. The number of records retrieved from the county crop acreage file was different for each compound, crop, and county because a pesticide use-coefficient did not exist for every compound and crop combination. This retrieval resulted in a file of many records for each county, each record representing one compound and crop combination. The final file-the county pesticide use file-contained more than 516,000 records. Each record included a five-digit state and county FIPS code, crop name, crop acreage, pesticide name, pesticide code, and pesticide type.

To complete the calculation of the amount of compound applied and the area treated, a second key, STPESTCROP, was created to link the new county pesticide use file to the state pesticide use file. This key comprises three numeric codes that are common to both the crop acreage file and the state pesticide use file and includes the two-digit state FIPS code (ST), fourdigit pesticide code (PEST), and the three-digit crop code (CROP). Using the STPESTCROP key, the county pesticide use file was temporarily linked to the 
state pesticide use file. The key allowed records in the two files to be matched by the state, the pesticide code, and the crop code, thereby ensuring that the correct use-coefficients were used to calculate the county pesticide use information. The two use-coefficients include the percentage of area treated and the average rate of annual application for each state, compound, and crop combination. These two pesticide use-coefficients were applied to each reported crop and compound combination in a county:

$$
\begin{aligned}
& \text { County Pesticide Use by Compound } \\
& \quad=\text { crop area } \\
& \times \text { percentage of area treated } \\
& \times \text { amount of active ingredient }
\end{aligned}
$$

\section{LIM ITATIONS OF THE COUNTY PESTICIDE USE DATA FOR WATER QUALITY ASSESSMENT}

National and regional estimates of pesticide use can be determined from the state data that were produced by NCFAP or from the county estimates derived using NCFAP's pesticide use-coefficients and county crop data. However, both the state and county data have limitations. The pesticide use-coefficients developed by NCFAP are based on statewide averages of both application rates for a compound applied to a crop and the average percentage of a crop's area treated with a compound; therefore, the data do not yield precise estimates of pesticide use at the county level. In addition, the state use-coefficients do not reflect the local variability of cropping and management practices found within many states and counties. Even though the crop area that is used in the calculations of pesticide use are taken from the 1992 Census, the pesticide use-coefficients were developed from data collected over a 4year period. Therefore, the use estimates do not represent a specific year, but rather, general patterns of use over a 4-year period.

Pesticide use-coefficients were developed only for those states located within the conterminous United States, and therefore, pesticide use for the Hawaiian Islands and Alaska cannot be estimated. Many specialty crops are grown on the Hawaiian Islands, such as sugar cane and vegetables, many of which are treated with a variety of pesticide compounds; pesticide use, however, cannot be calculated because use-coefficients are not published for the crops grown or the pesticide used on the Hawaiian Islands.
Another potential factor that affects estimates derived from these data is the result of Census nondisclosure rules. The Census does not publish information that can be related to the operation of an individual farm. For example, the Census does not publish information from counties where three or fewer farms reported acreage for a specific crop, such as rice. Therefore, it would not be possible to estimate county use for any of the pesticides applied to a crop with nondisclosed acreage because county crop acreage is a main component in the calculation of pesticide use (crop area $\times$ percentage of crop treated $\times$ average amount of an active ingredient applied). In these instances, pesticide usage can be underestimated. Estimates of pesticides applied to pasture may also be underestimated because the only readily available source of pasture area is available from the Census, and the Census does not include information on federally owned land that is used for pasture or grazing.

\section{SUMMARIES OF PESTICIDE USE}

The county pesticide-use data were used to summarize national use by pesticide compound, to rank pesticide use by individual crop, to "calculate total" pesticide use for each of the NAWQA study units, and to produce estimates for ground-water and surfacewater studies within the study unit. Table 3 ranks each of the 208 compounds according to the estimated total application in pounds. The data were produced in two steps. First, the amounts of each compound applied to all crops in each county were summed to produce totals for each compound. Second, the county totals were summed nationally for each compound. The compounds were then ranked in order of total application nationwide. The ranking was influenced by both the amount of a compound applied to crop (recommended application rate) and the amount of total crop area.

The application totals by active ingredient, shown in table 3, are not in exact agreement with the national totals published by NCFAP. The totals listed in table 3 are based on use-coefficients that are applied to county acreage, whereas the NCFAP totals are based on applying the coefficients to state acreage estimates from the 1992 Census. This difference can be explained by the instances in which the Census does not publish county-level estimates for a crop, but includes the county acreage in the state total. Thus, the national estimates summarized from the county data in table 3 usually are lower than the NCFAP estimates. 
Table 4 lists the pesticide compounds and the primary crops associated with each pesticide in order of highest to lowest percentage of application. Only crops that represent at least 5 percent of the national total use are listed. Table 4 shows the diversity of crops that each compound is applied to and the variety of compounds used to treat a particular crop.

\section{THE NATIONAL W ATER-QUALITY ASSESS- MENT'S STUDY UNIT SUM MARIES}

Table 5 summarizes pesticide use for each NAWQA study unit. For each study unit, all compounds are included that represent at least 1 percent of pesticide use within the study unit. The amount applied, area treated, percentage of national use, percentage of United States area treated, average amount applied annually to cropland and pasture, percentage of use within the study unit, and percentage of total use among the study units are shown. The average application rate over the entire study unit provides regionalscale use intensity (that is, intensity of application) throughout the basin, whereas average annual application to cropland and pasture represents use intensity in agricultural areas.

To produce the study unit summaries of pesticide use presented in table 5, a different approach was taken than that used to produce county totals and national estimates of pesticide use. Pesticide estimates were needed for the study unit area only, and so, it was necessary to exclude the area of each county located outside the study unit boundary. Using a GIS, a polygon coverage of NAWQA study-unit boundaries was overlaid with a $1: 100,000$-scale map of the counties contained within the conterminous United States. The overlay process created a new polygon coverage that included the intersection of county and study-unit boundaries. NAWQA study-unit boundaries are irregular and sometimes do not follow county boundaries; therefore, each of the polygons created from the overlay process contains more than one record of attribute information. The attribute information includes the area of each polygon (reported in square meters), a FIPS code from the county coverage, and a study unit name if the polygon fell within the study-unit boundary. Multiple records in the resultant polygon coverage attribute table have the same county FIPS code and name. Using Steel County as an example of this overlay process, three of these records represent areas of Steele County, Minn., that are located inside the east- ern Iowa study unit, one record is the county area located within the upper Mississippi study unit, and another three records are based on regions of the county that are located outside any of the study-unit boundaries. By summing the county area associated with each of the records for a county-in this case, the seven records representing Steele County-the total area of a county was calculated. For example, figure 2 displays seven records that are associated with Steele County (FIPS 27147), and figure 3 shows a map of counties located within the eastern Iowa study unit. The area of a county located within a study unit was calculated using records associated with both the county and the study unit. From the area totals (total county area and county area within the study unit), a county weighting factor was calculated by dividing the area of a county within the study unit by the total county area. The weighting factor was important because it was used to proportion the estimated acres treated with pesticides and the amount applied for those counties that were not entirely contained within a study unit.

Using the county weighting factor to calculate the area and quantity of a pesticide's use assumes that the crop acreage and the compound's application are evenly distributed across a county. Where agricultural patterns are localized within a county, this procedure can produce overestimates or underestimates of pesticide use, depending on the collocation of agricultural land and the study-unit boundary. In order to develop estimates of pesticide use that are based on the distribution of agricultural land within the county or a basin, a crop weighting factor was developed by overlaying maps of agricultural land use with maps of county boundaries and basin boundaries (Naomi Nakagaki, unpub. data, 2000). Figure 4 shows mapped USGS land-use and land-cover information for Sumter County, Ga. (FIPS 13261), overlaid with a NAWQA basin boundary. Within Sumter County, 168,304 acres are mapped as cropland and pasture; of this total, 26,362 acres are located within the NAWQA basin boundary. Cropland and pasture area (26,362 acres) located within the NAWQA basin was divided by total cropland and pasture within the county (168,304 acres) to produce a cropland and pasture weighting factor for the basin, which in this case is approximately 15 percent. The crop weighting factor was applied to county pesticide use data to estimate pesticide use for the basin. In this example, an estimated 20,168 pounds of pesticides was applied throughout Sumter County; 


\begin{tabular}{|c|c|c|c|c|c|c|}
\hline FTS & $\begin{array}{c}\text { County } \\
\text { Name }\end{array}$ & $\begin{array}{c}\text { Polygon } \\
\text { Area } \\
\left(\mathrm{m}^{2}\right)\end{array}$ & $\begin{array}{c}\text { Study } \\
\text { Unit }\end{array}$ & $\begin{array}{c}\text { Total County } \\
\text { Area } \\
\left(\mathrm{m}^{2}\right)\end{array}$ & $\begin{array}{c}\text { County Area } \\
\text { in Study Unit } \\
\left(\mathrm{m}^{2}\right)\end{array}$ & $\begin{array}{c}\text { Percent County } \\
\text { Area }\end{array}$ \\
\hline 27147 & Steele & $17,682,910$ & & $1,119,253,803$ & & 1.50 \\
\hline 27147 & Steele & $1,030,417,000$ & UMIS & $1,119,253,803$ & & 92.06 \\
\hline 27147 & Steele & 261,732 & & $1,119,253,803$ & & 0.02 \\
\hline 27147 & Steele & $51,352,500$ & & $1,119,253,803$ & & 1.74 \\
\hline 27147 & Steele & $19,316,670$ & EIWA & $1,119,253,803$ & $19,539,661$ & \\
\hline 27147 & Steele & 183,198 & EIWA & $1,119,253,803$ & $\mathbf{A}$ & \\
\hline 27147 & Steele & 39,793 & EIWA & $1,119,253,803$ & & \\
\hline
\end{tabular}

Figure 2. Seven records associated with Steele County, M innesota, which display the area of the county associated with two National Water-Quality Assessment Program study units

however, using the crop weighting factor, the revised estimate is 3,158 pounds of pesticides applied within the NAWQA basin. Applying the weighting factor to individual compounds can further refine the pesticide use estimate. For example, county use of alachlor is estimated to be 5,985 pounds, but if the crop weighting factor is applied, alachlor use within the NAWQA basin is 917 pounds. If information on the acreage and location of specific crops were available, then the usecoefficients, the percentage of area treated, and the average amount applied, could be used instead of assuming that specific crops are distributed evenly throughout the county, as is the case with maps of cropland and pasture.

\section{MAP DISPLAYS OF PESTICIDE USE DATA}

Pesticide-use patterns were also mapped using a GIS. County pesticide-use data provide information for counties that have applications of pesticides along with the corresponding rate of use, but from these data alone, the spatial distribution and intensity of use are not apparent. A GIS with a relational database is the tool to create maps that display tabular data spatially.

A digital map of United States counties and pesticide use information stored in a relational database were linked using the five-digit FIPS code - an element that was common to both data sets. The attribute file for the county coverage contained the FIPS code, name, and area of each county. Three additional items were added to the county attribute file: area of the county in cropland and pasture, the amount of a com- pound applied per unit area of the county, and the amount of compound applied per unit area of cropland and pasture. By selecting a compound name or pesticide code, all the records for that compound were selected from the pesticide file. By linking the digital map with the pesticide file using the FIPS code, other information in each of the files could be used. For example, the amount of a compound in the pesticide file could be divided by the area of the county in a map attribute file to estimate amount of pesticide use in pounds applied per acre. By adding the conversion from square meter to acre to the calculation, the map could be displayed as pounds per acre. Similarly, the amount applied per unit area of cropland and pasture could be calculated by linking the Census county data to the pesticide data using the 5-digit FIPS code. Figures 5 and 6 show the distribution and intensity of use across the conterminous United States for four frequently used pesticides. Figure 5 shows the average amount (in pounds) applied per square mile for two herbicides- acethochlor and metolachlor; figure 6 shows use of the fumigant methyl bromide and the insecticide chlorpyrifos. Each map displays, in four intervals, the amount of active ingredient applied over a county. The four class intervals were established independently for each of the compounds for counties that reported use of the specific compound (greater than zero pounds applied per acre). Each class interval represents an equal number of data values from the distribution of the pesticide data, with the first interval representing the 25 th percentile, the second representing the 50th percentile (or the median), and the third interval representing the 75 th percentile. 


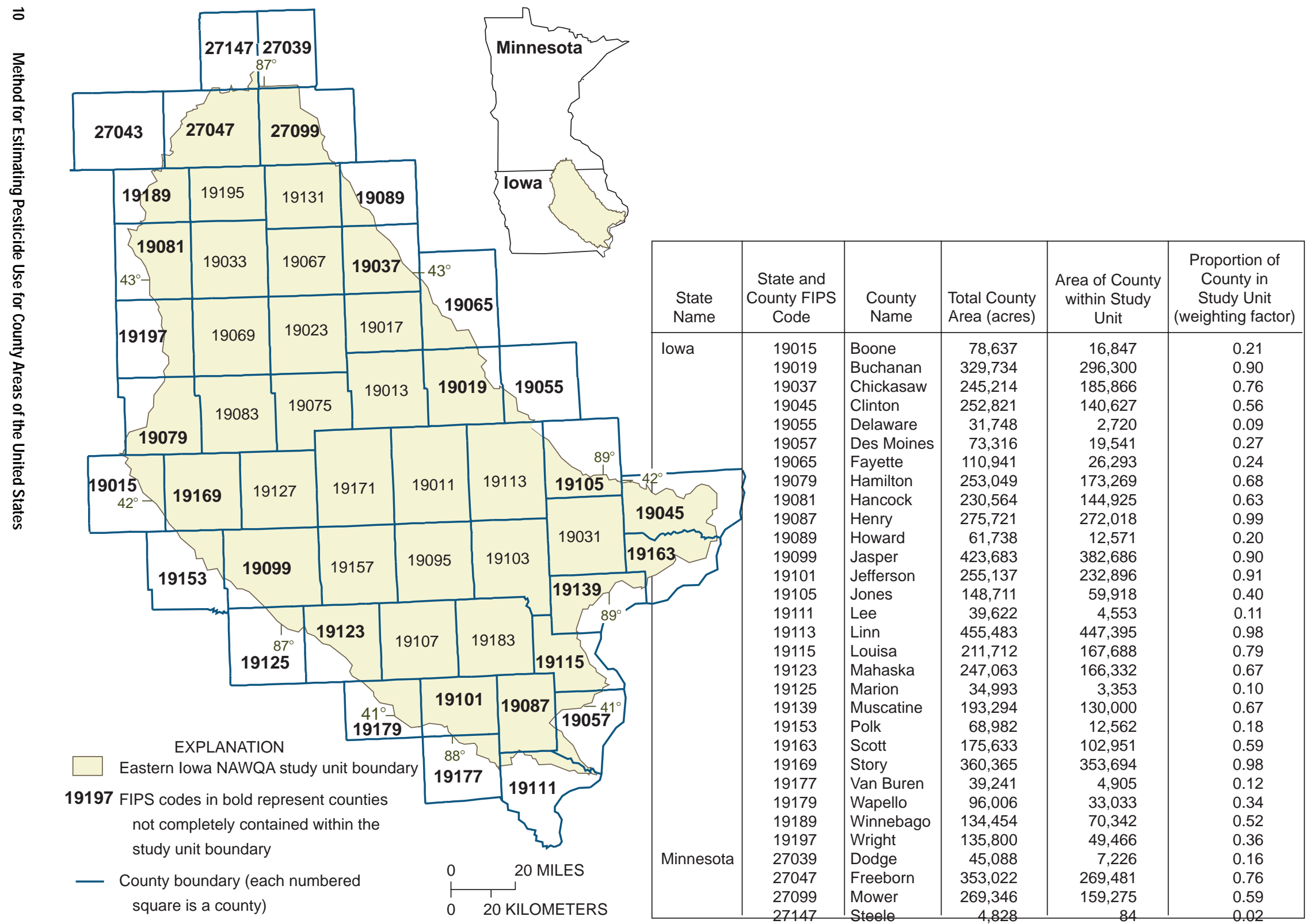

Figure 3. Eastem lowa Study Unit. 


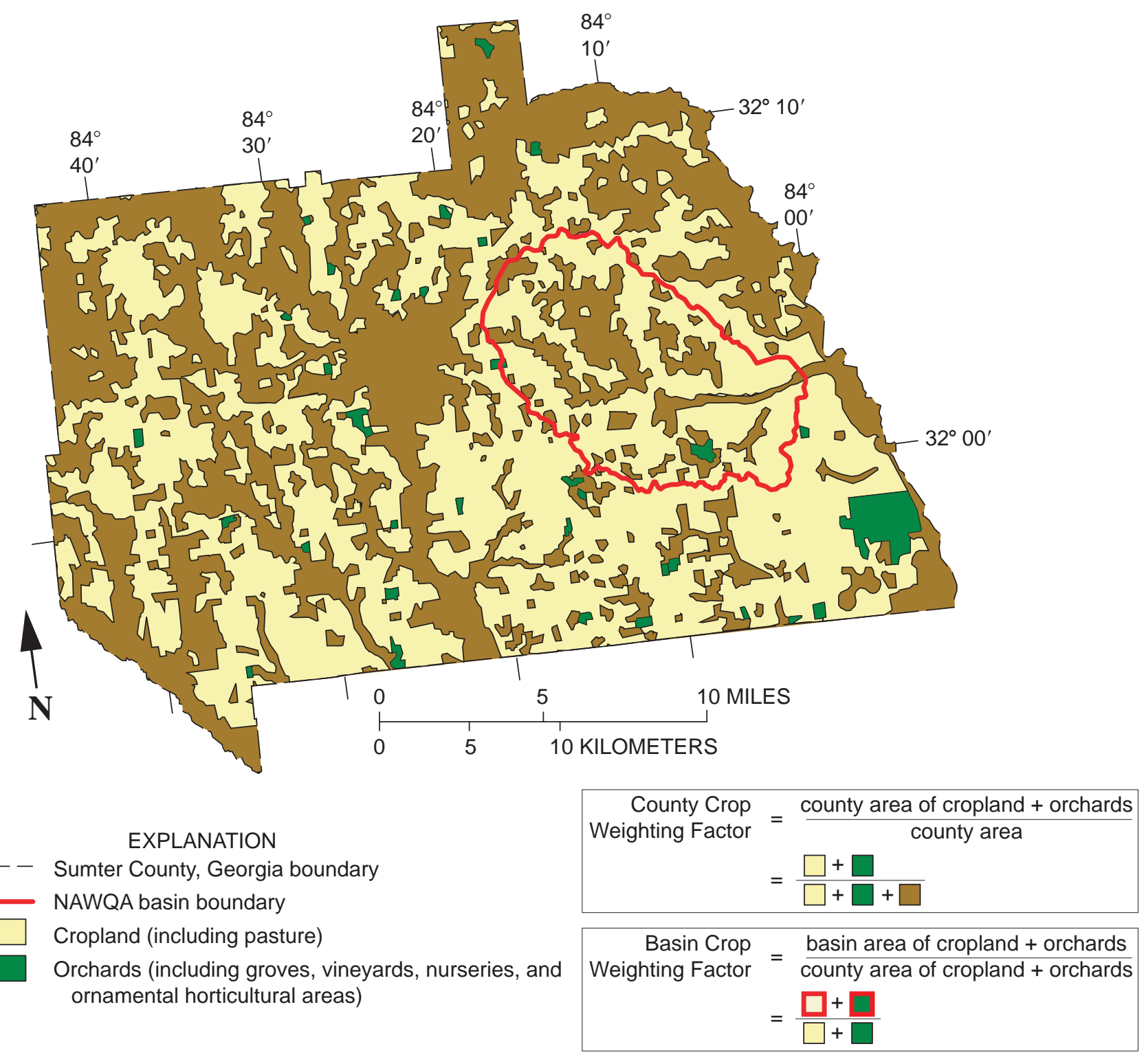

Figure 4. Calculating a crop weighting factor for a county and a basin. 

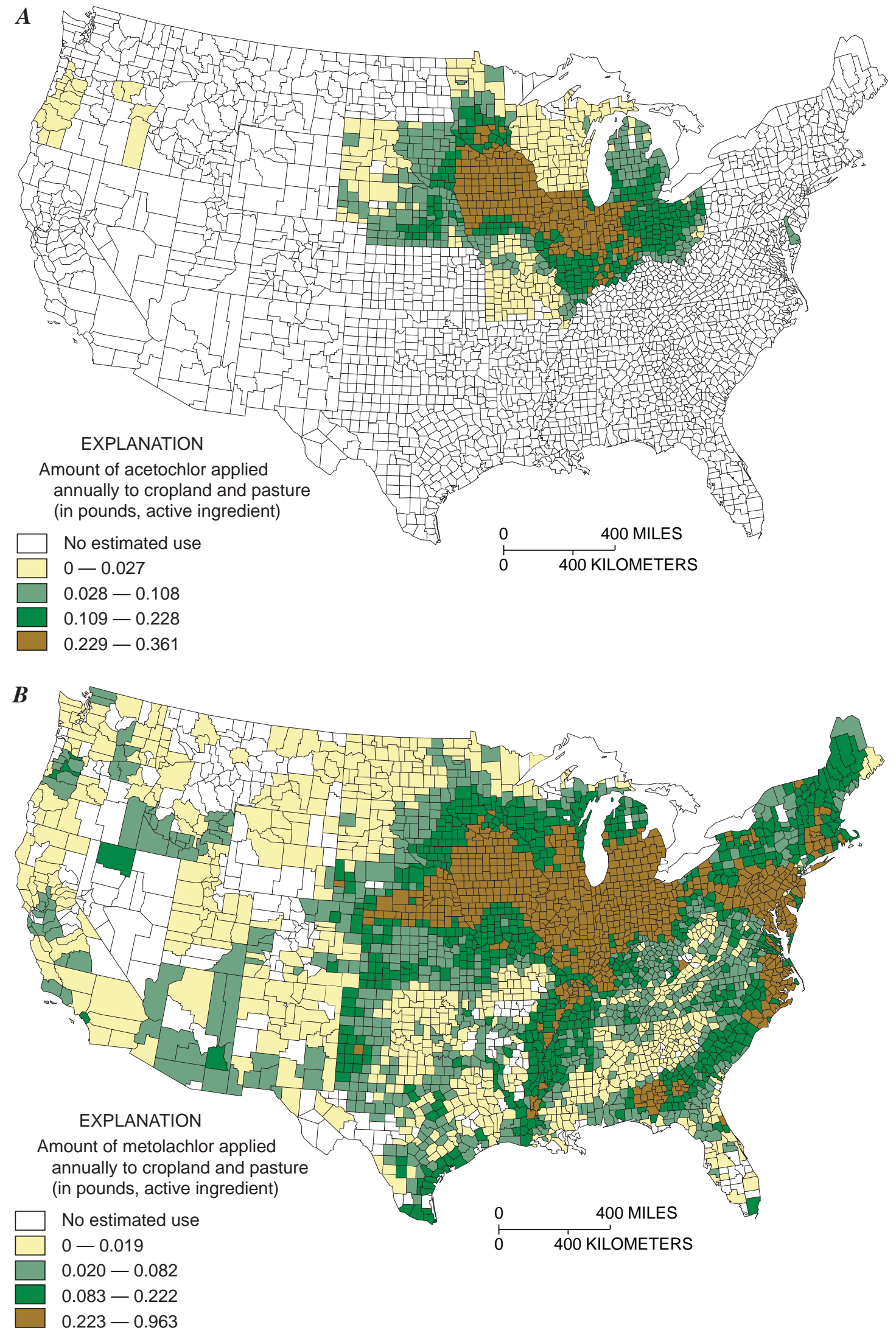

Flgure 5. Average annual amount of active ingredient of acetochlor and metolachlor applied to cropland and pasture area in a county 


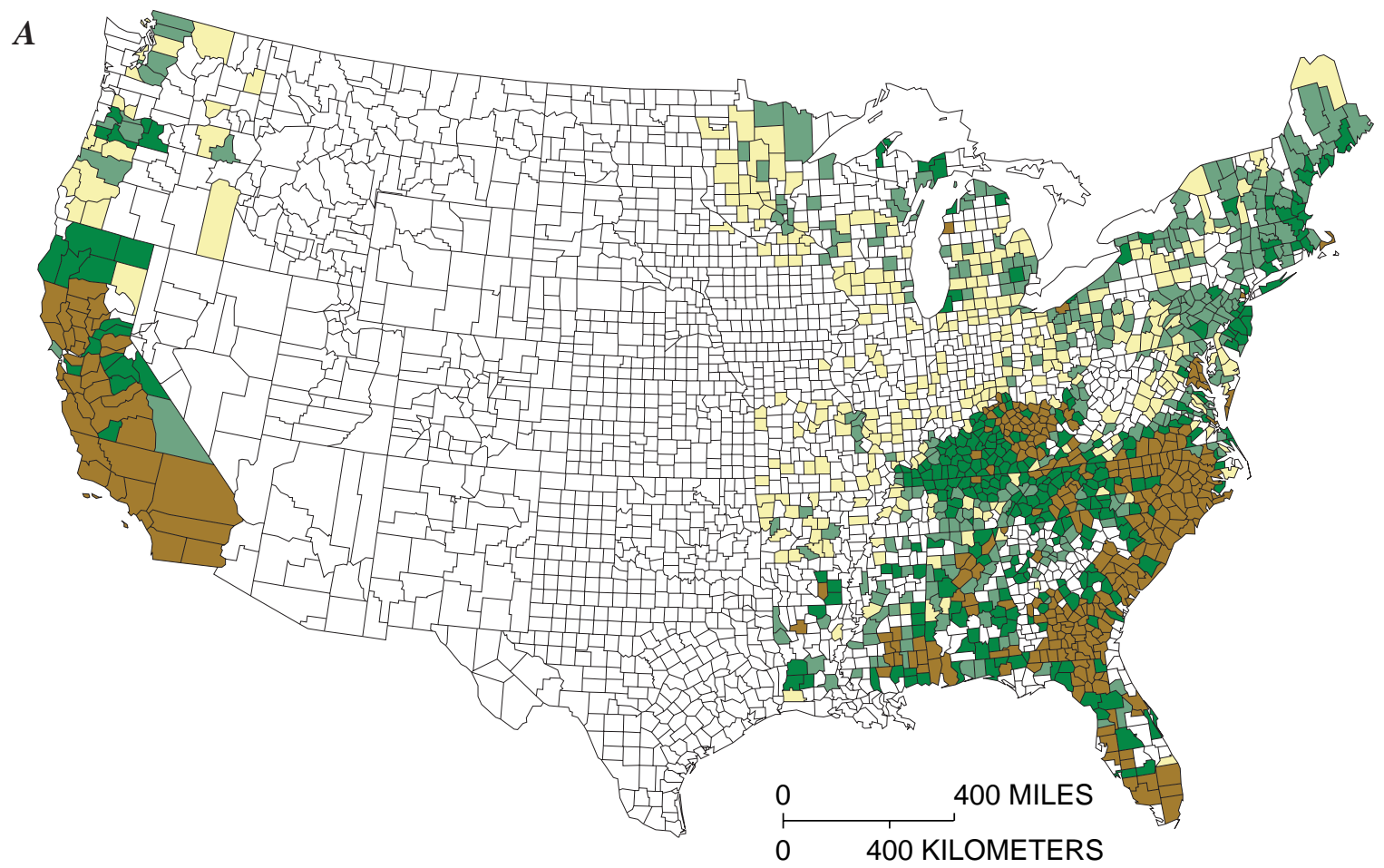

EXPLANATION

Amount of methyl bromide applied annually to cropland and pasture (in pounds, active ingredient)

No estimated use

$0-0.007$

$0.008-0.042$

$0.043-0.245$

$0.246-60.056$

\begin{tabular}{|l|c|c|}
\hline \multicolumn{1}{|c|}{ Crops } & $\begin{array}{c}\text { Total Amount Applied } \\
\text { (in pounds, active ingredient) }\end{array}$ & $\begin{array}{c}\text { National Use } \\
\text { (in percent) }\end{array}$ \\
\hline tomatoes & $13,035,891$ & 33.22 \\
tobacco & $5,603,849$ & 14.28 \\
strawberries & $5,629,716$ & 14.09 \\
watermelons & $3,748,578$ & 9.55 \\
sweet peppers & $3,140,497$ & 8.00 \\
grapes & $2,668,467$ & 6.79 \\
almonds & $1,697,323$ & 4.32 \\
carrots & 607,371 & 1.55 \\
nectarines & 478,112 & 1.22 \\
cucumbers & 406,998 & 1.04 \\
\hline
\end{tabular}

Figure 6. Average annual amount of active ingredient of methyl bromide (A) and chlorpyrifos (B) applied to cropland and pasture area in a county. 


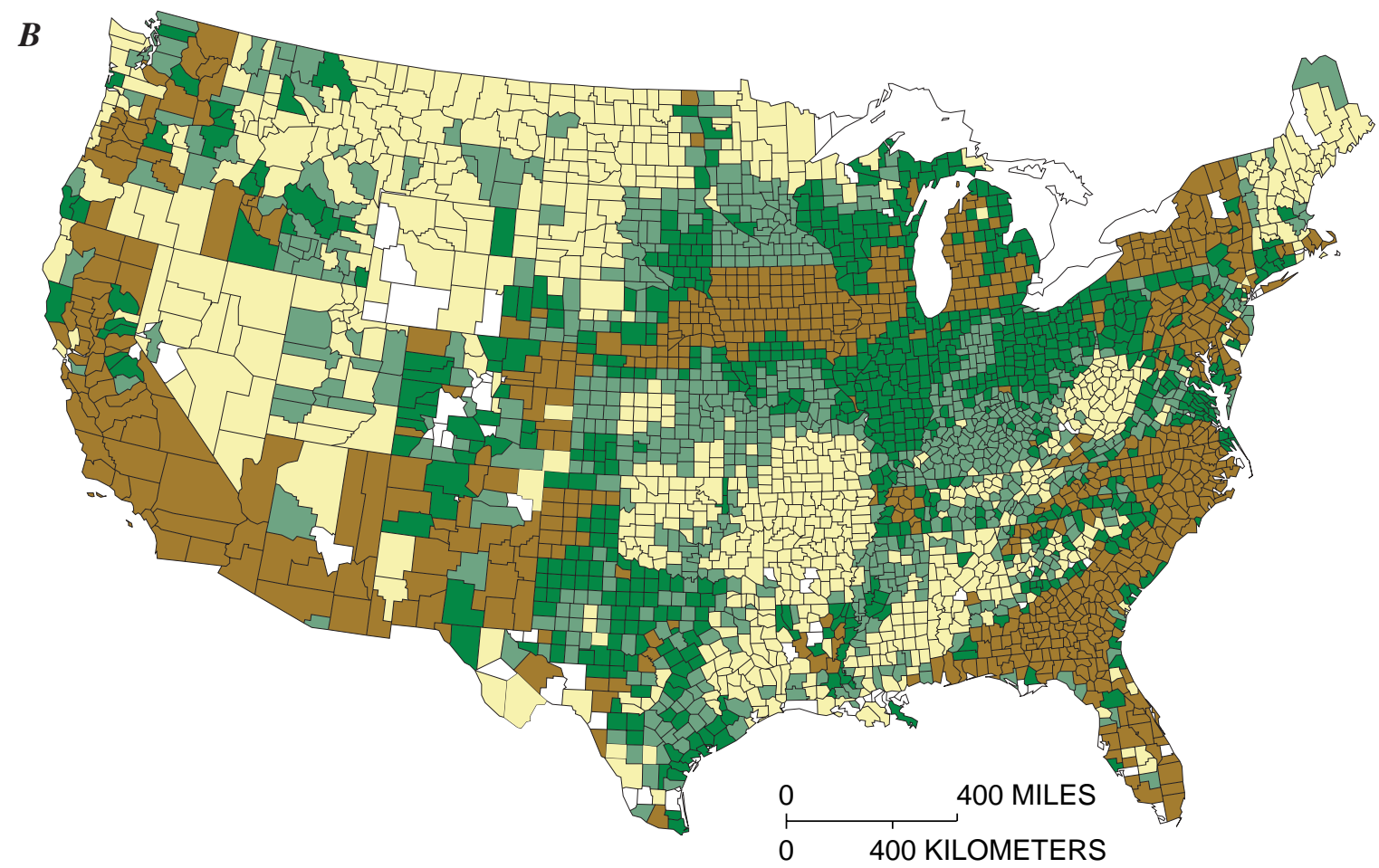

EXPLANATION

Amount of chlorpyrifos applied annually to cropland and pasture (in pounds, active ingredient)

No estimated use

$0-0.010$

$0.011-0.023$

$0.024-0.047$

$0.048-0.858$

\begin{tabular}{|l|c|c|}
\hline \multicolumn{1}{|c|}{ Crops } & $\begin{array}{c}\text { Total Amount Applied } \\
\text { (in pounds, active ingredient) }\end{array}$ & $\begin{array}{c}\text { National Use } \\
\text { (in percent) }\end{array}$ \\
\hline corn & $6,710,506$ & 43.55 \\
cotton & $1,616,065$ & 10.49 \\
peanuts & 912,717 & 5.92 \\
alfalfa hay & 872,215 & 5.68 \\
wheat and grains & 741,185 & 4.81 \\
tobacco & 706,200 & 4.60 \\
all citrus & 675,060 & 4.38 \\
apples & 654,938 & 4.25 \\
\hline
\end{tabular}

Figure 6. Average annual amount of active ingredient of methyl bromide (A) and chlorpyrifos (B) applied to cropland and pasture area in a county-Continued. 


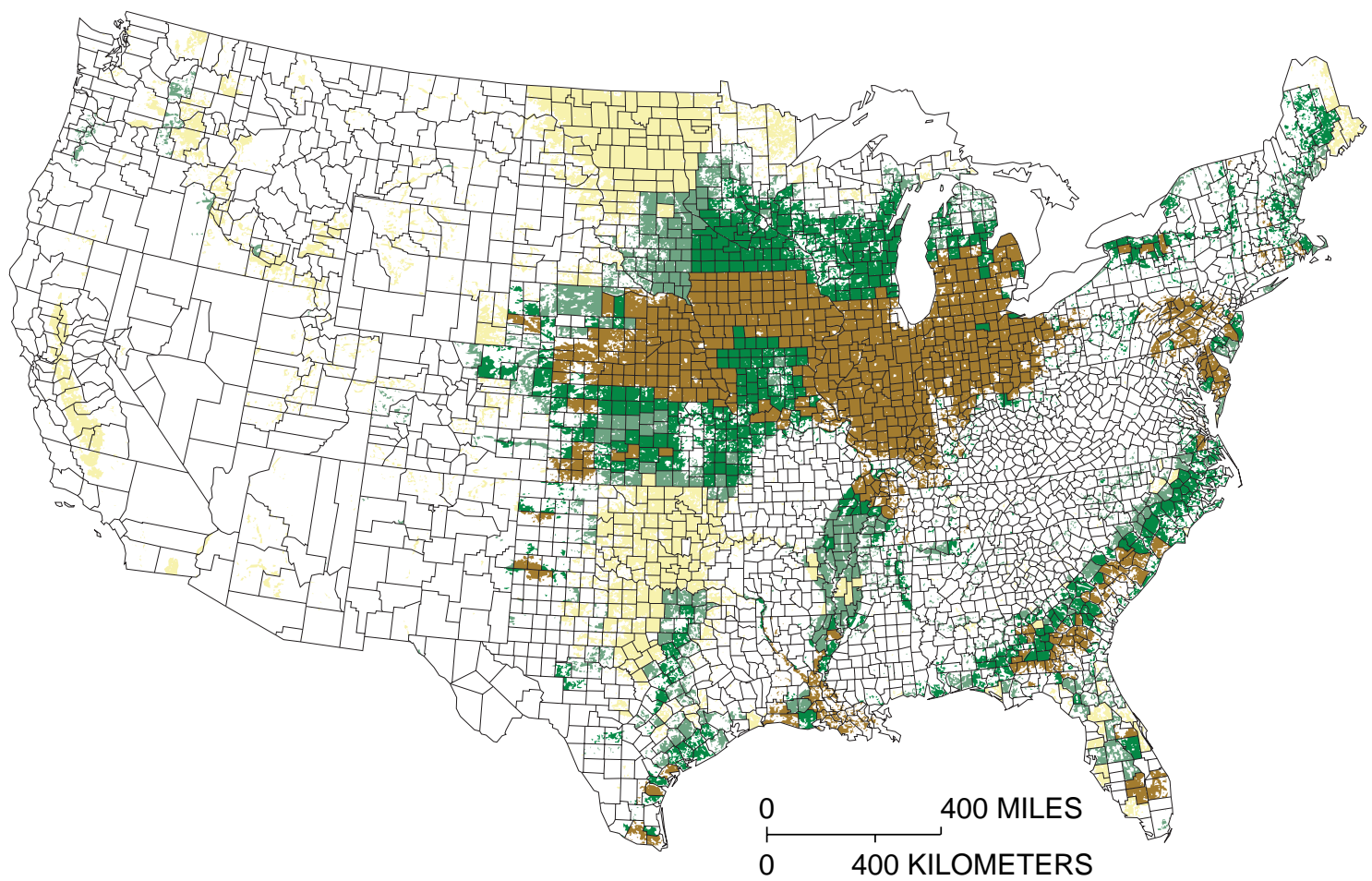

\section{EXPLANATION}

Amount of atrazine applied annually to cropland and pasture (in pounds, active ingredient)

No estimated use

$<0.02$

$0.03-0.11$

$0.12-0.25$

$0.26-2.11$

\begin{tabular}{|l|c|c|}
\hline \multicolumn{1}{|c|}{ Crops } & $\begin{array}{c}\text { Total Amount Applied } \\
\text { (in pounds, active ingredient) }\end{array}$ & $\begin{array}{c}\text { National Use } \\
\text { (in percent) }\end{array}$ \\
\hline corn & $53,796,206$ & 84.13 \\
sorghum & $7,339,963$ & 11.48 \\
sugar cane: sugar \& seed & $1,711,322$ & 2.68 \\
pasture & 518,074 & 0.81 \\
sweet corn & 444,523 & 0.70 \\
sod & 119,182 & 0.19 \\
proso millet & 11,937 & 0.02 \\
field and grass seed & 6,305 & 0.01 \\
\hline
\end{tabular}

Figure 7. Average annual atrazine use applied to cropland categories mapped from Advanced Very High Resolution Radiometer data. 
The maps shown in figures 5 and 6 represent use of a pesticide as equally distributed across a county. Display of the pesticide use information can be improved by using other ancillary data. For example, using the GIS, maps of land use and land cover can be linked to the pesticide use data in the same manner as the county maps were linked to the study unit boundaries. In the example in figure 7, a national classification of land-cover was used to map the distribution of the compound atrazine in a county. The land-cover classification was derived from satellite data collected by the Advanced Very High Resolution Radiometer (AVHRR) (Loveland and others, 1991). The land-cover classification contained over 150 classes, 50 of which were related to agricultural land use. The individual agricultural land-cover categories were combined into a single category. As part of each map (figs. 6 and 7), a table lists, in order of use, the crop treated with the compound, the total amount (in pounds) of the active ingredient applied to the crop, and the percentage of national use. Because the percentages are rounded, they do not always total 100 percent.

County boundaries that included a FIPS code were overlaid on the land-cover classification using a GIS map overlay function. The land-cover map could now be referenced to a FIPS code and linked to the pesticide data using the FIPS code. The area of mapped agriculture in a county could be totaled, and in this case, converted to acres, and used as the denominator to calculate the amount of an active ingredient (atrazine) applied to each acre of agricultural land use. Figure 7 shows a map of atrazine use applied to agricultural land mapped from AVHRR data.

The addition of other spatial and tabular data could be used to further refine both the estimates of pesticide use and their distribution at state and regional levels. The greatest improvement in estimating pesticide usage would be obtained by incorporating information on the location and amount of specific crops grown and crop rotation practices. However, this more detailed spatial information is not readily available. Regional use-coefficients could be developed using information on crop management practices and crop requirements, such as irrigation, tillage, the number and timing of pesticide applications, crop phenology, soils, and climate. These additions could provide a temporal component and could be used to estimate timing and concentrations of pesticide loads in surface water. These refinements are needed to better characterize environmental risk and exposure and to model the transport of pesticide residues through the hydrologic system.

\section{SUMMARY}

County average annual pesticide-use data have been compiled for 208 compounds used in crop production in the United States. The U.S. Geological Survey's National Water-Quality Assessment (NAWQA) Program is using these data to estimate pesticide concentrations and loads for areas not sampled by the study units and to develop and explain the occurrence of pesticides in surface and ground waters in relation to estimated use and other physical requirements such as soil and climate. The county data are based on pesticide use rates compiled by the National Center for Food and Agriculture Policy (NCFAP) from pesticide use information collected by state and federal agencies over a 4year period (1992-1995) and on crop acreage data from the 1992 Census of Agriculture. The NCFAP database contains state-based estimates of pesticideuse rates for 208 compounds and 86 crops. For each of the compounds, two use-coefficients were developed: the percentage of area treated for 86 specific crops and the average amount of an active ingredient applied annually to each acre of that crop.

The state-based pesticide-use coefficients were applied to county crop acreage obtained from the 1992 Census. County estimates of pesticide use were derived for over 16,000 possible compound and crop combinations found throughout the 3,067 counties within the conterminous United States that reported crop acreage. Crop acreages for each county were retrieved from the Census. To calculate county pesticide use by compound and crop, the county crop acreage was combined with the percentage of each crop's area that was treated and with the average annual application rate of the active ingredient.

The resulting county pesticide database provides a comprehensive source of information on the application of more than 200 compounds used in crop production in the United States. The data provide a single source of information on pesticide use that is consistent in both scale and level of detail, thus, allowing regional comparisons of pesticide use to be made. The data have been aggregated to provide information for the nation and for NAWQA study units. These data provide a means to assess regional and national relationships between pesticide use and water quality. 


\section{REFERENCES}

Gianessi, L.P. and Anderson, J.E., 1995, Pesticide use in U.S. crop production, national summary report: Washington, D.C., National Center for Food and Agricultural Policy, variously paged.

Gilliom, R.J. and Thelin, G.P., 1997, Classification and mapping of agricultural land for national water-quality assessment: U.S. Geological Survey Circular 1131, $70 \mathrm{p}$.

Gilliom, R.J., Alley, W.M., and Gurtz, M.E., 1995, Design of the National Water-Quality Monitoring Program: Occurrence and distribution of water-quality conditions: U.S. Geological Survey Circular 1112, 33 p.
Hitt, K.J., 1994, Refining 1970's land-use data with 1990 population data to indicate new residential development: U.S. Geological Survey Water-Resources Investigations Report 94-4250, 15 p.

Loveland, T.R., Merchant, J.W., Ohlen, D.O., and Brown, J.F., 1991, Development of a land-cover characteristics database for the conterminous U.S.: Photogrammetric Engineering \& Remote Sensing, v. 57, no. 11, p. $1453-1463$.

U.S. Department of Commerce, 1995, 1992 Census of agriculture. Geographic area series 1A. U.S. summary state data file, and cross-tab data file: U.S. Department of Commerce, Bureau of the Census, Data User Services Division, CD-AG-92-1C [CD-ROM, dBase format]. 
Table 2. Crop codes and crop names with corresponding Census of Agriculture data codes

\begin{tabular}{|c|c|c|c|c|c|}
\hline $\begin{array}{l}\text { Crop } \\
\text { code }\end{array}$ & Crop & $\begin{array}{l}\text { Census of Agriculture } \\
\text { Data Code }\end{array}$ & $\begin{array}{l}\text { Crop } \\
\text { code }\end{array}$ & Crop & $\begin{array}{l}\text { Census of Agriculture } \\
\text { Data Code }\end{array}$ \\
\hline 653 & Alfalfa Hay & 280132 & 119 & Melons & 290106 \\
\hline 108 & Almonds & 310317 & 660 & Millet & 260092 \\
\hline 701 & Apples & 310002 & 910 & Mint & 340057 \\
\hline 122 & Apricots & 310011 & 112 & Nectarines & 310137 \\
\hline 102 & Artichokes & 290010 & 533 & Oats & 260082 \\
\hline 116 & Asparagus & 290014 & 152 & Okra & 290134 \\
\hline 123 & Avocados & 310020 & 135 & Olive & 310146 \\
\hline 535 & Barley & 260042 & 709 & Onions & 290126 \\
\hline 128 & Beets & 290026 & 654 & Other Hay & $\begin{array}{l}280127 \text { (Other Hay) - } \\
280132 \text { (Alfalfa Hay) }\end{array}$ \\
\hline 915 & Blackberries & 320008 & 502 & Parsley & 290138 \\
\hline 400 & Blueberries & $\begin{array}{l}320012 \text { (Tame Blueberries) } \\
\text { + } 320017 \text { (Wild Blueberries) }\end{array}$ & 842 & Pasture & 60073 \\
\hline 109 & Broccoli & 290030 & 702 & Peaches & 310173 \\
\hline 100 & Brussel Sprouts & 290034 & 690 & Peanuts & 270077 \\
\hline 716 & Cabbage & 290042 & 703 & Pears & 310182 \\
\hline 680 & Canola & 260057 & 105 & Pecans & 310344 \\
\hline 715 & Cantaloupes & 290050 & 136 & Pistachios & 310353 \\
\hline 714 & Carrots & 290054 & 120 & Plums & 310200 \\
\hline 110 & Cauliflower & 290058 & 244 & Pomegranates & 310209 \\
\hline 713 & Celery & 290062 & 717 & Potatoes & 270042 \\
\hline 704 & Cherries & 310038 & 143 & Pumpkins & 290158 \\
\hline 700 & Citrus & 310227 & 147 & Radishes & 290162 \\
\hline 118 & Collards & 290074 & 236 & Raspberries & 320042 \\
\hline 530 & Corn & $\begin{array}{l}260002 \text { (Corn for Grain) + } \\
280157 \text { (Corn for Silage) }\end{array}$ & 605 & Rice & 260097 \\
\hline 520 & Cotton & 270002 & 139 & Rye & 260102 \\
\hline 914 & Cranberries & 320027 & 140 & Safflower & 260107 \\
\hline 712 & Cucumbers & 290082 & 949 & Sod & 330043 \\
\hline 160 & Dates & 310083 & 570 & Sorghum & $\begin{array}{l}260007(\text { Grain })+ \\
280162(\text { Dry Forage })+ \\
280167(\text { Silage })\end{array}$ \\
\hline 800 & Dry Beans & $\begin{array}{l}270017 \text { (Edible Dry Beans) } \\
+270022 \text { (Dry Lima Beans) } \\
+270037 \text { (Lentils) }\end{array}$ & 600 & Soybeans & 270012 \\
\hline 801 & Dry Peas & $\begin{array}{l}270027 \text { (Edible Dry Peas) + } \\
270032 \text { (Dry Cowpeas) }\end{array}$ & 126 & Spinach & 290170 \\
\hline 150 & Eggplant & 290090 & 127 & Squash & 290174 \\
\hline 401 & $\begin{array}{l}\text { Field and Grass } \\
\text { Seed }\end{array}$ & 280002 & 103 & Strawberries & 320047 \\
\hline 145 & Figs & 310092 & 107 & Sugar Beets & 270057 \\
\hline 913 & Filberts & 310326 & 952 & Sugarcane & $\begin{array}{l}270062 \text { (Sugar Cane for Seed) }+ \\
270067 \text { (Sugar Cane for Sugar) }\end{array}$ \\
\hline 943 & Flax & 260072 & 121 & Sunflowers & 260112 \\
\hline 117 & Garlic & 290102 & 707 & Sweet Corn & 340092 \\
\hline 111 & Grapes & 310101 & 101 & Sweet Peppers & 290150 \\
\hline 711 & Green Beans & $\begin{array}{l}290018 \text { (Lima Beans) + } \\
290022 \text { (Snap Beans) }\end{array}$ & 158 & Sweet Potatoes & 270047 \\
\hline 245 & Green Onions & 290130 & 670 & Tobacco & 270007 \\
\hline 104 & Green Peas & $\begin{array}{l}290078 \text { (Cowpeas) + } \\
290142 \text { (Green Peas) }\end{array}$ & 706 & Tomatoes & 290182 \\
\hline 911 & Hops & 340037 & 115 & Walnuts & 310362 \\
\hline 941 & Hot Peppers & 290146 & 705 & Watermelons & 290202 \\
\hline 243 & Kiwi & 310119 & 540 & Wheat & 260012 \\
\hline 710 & Lettuce & 290118 & 900 & Wild Rice & 260122 \\
\hline
\end{tabular}


Table 3. Summary of national pesticide use, 1992-1995, derived from county pesticide use information sorted by total application in pounds active ingredient in descending order

\begin{tabular}{|c|c|c|c|c|}
\hline Compound & $\begin{array}{l}\text { Pesticide } \\
\text { code }\end{array}$ & Pesticide type & $\begin{array}{l}\text { Total application, (pounds, } \\
\text { active } \\
\text { ingredient) }\end{array}$ & $\begin{array}{l}\text { Total area treated } \\
\text { (acres) }\end{array}$ \\
\hline Sulfur & 5004 & Fungicide & $86,750,257$ & $2,471,066$ \\
\hline Atrazine & 1980 & Herbicide & $63,947,513$ & $56,985,754$ \\
\hline Metolachlor & 1011 & Herbicide & $57,932,590$ & $31,262,371$ \\
\hline Oil & 6049 & Insecticide & $53,184,324$ & $1,354,858$ \\
\hline $1,3-\mathrm{D}$ & 8008 & Other Pesticides & $40,023,187$ & 492,847 \\
\hline Methyl Bromide & 8001 & Other Pesticides & $39,246,466$ & 204,350 \\
\hline 2,4-D & 1302 & Herbicide & $37,238,146$ & $77,794,014$ \\
\hline Metam Sodium & 8002 & Other Pesticides & $32,237,173$ & 383,881 \\
\hline Cyanazine & 1369 & Herbicide & $29,535,834$ & $15,846,727$ \\
\hline Sulfuric Acid & 8016 & Other Pesticides & $25,941,349$ & 105,608 \\
\hline Alachlor & 1863 & Herbicide & $25,647,684$ & $14,529,371$ \\
\hline Acetochlor & 3000 & Herbicide & $23,841,035$ & $11,845,501$ \\
\hline Pendimethalin & 1629 & Herbicide & $20,723,987$ & $21,498,031$ \\
\hline Trifluralin & 1361 & Herbicide & $19,786,700$ & $25,368,107$ \\
\hline Glyphosate & 1099 & Herbicide & $16,039,854$ & $23,821,020$ \\
\hline Chlorpyrifos & 6009 & Insecticide & $15,408,958$ & $13,955,168$ \\
\hline EPTC & 1414 & Herbicide & $14,097,139$ & $4,014,564$ \\
\hline Chlorothalonil & 5007 & Fungicide & $12,801,685$ & $3,137,717$ \\
\hline Copper & 5011 & Fungicide & $10,531,790$ & $2,505,811$ \\
\hline Dicamba & 1298 & Herbicide & $9,590,278$ & $38,967,795$ \\
\hline Methyl Parathion & 6042 & Insecticide & $8,930,188$ & $7,491,440$ \\
\hline Mancozeb & 5000 & Fungicide & $8,301,811$ & $2,043,763$ \\
\hline Butylate & 1839 & Herbicide & $7,701,494$ & $1,986,155$ \\
\hline Chloropicrin & 8000 & Other Pesticides & $7,443,548$ & 122,684 \\
\hline Propanil & 1282 & Herbicide & $7,229,829$ & $2,337,989$ \\
\hline Terbufos & 6060 & Insecticide & $7,200,077$ & $6,675,913$ \\
\hline Bentazon & 1287 & Herbicide & $6,729,259$ & $10,020,824$ \\
\hline MSMA & 1124 & Herbicide & $5,763,370$ & $3,807,455$ \\
\hline Sodium Chlorate & 8004 & Other Pesticides & $5,243,734$ & $1,711,868$ \\
\hline Carbofuran & 6007 & Insecticide & $5,124,406$ & $6,515,915$ \\
\hline Molinate & 1417 & Herbicide & $4,903,206$ & $1,420,255$ \\
\hline Simazine & 1981 & Herbicide & $4,810,327$ & $3,431,825$ \\
\hline MCPA & 1305 & Herbicide & $4,658,848$ & $12,316,934$ \\
\hline Carbaryl & 6006 & Insecticide & $4,554,089$ & $2,953,313$ \\
\hline Fluometuron & 1998 & Herbicide & $4,500,857$ & $4,983,276$ \\
\hline Aldicarb & 6003 & Insecticide & $4,282,750$ & $4,315,657$ \\
\hline Paraquat & 1616 & Herbicide & $4,228,555$ & $9,496,297$ \\
\hline Propachlor & 1191 & Herbicide & $3,865,533$ & $1,674,875$ \\
\hline Phorate & 6050 & Insecticide & $3,811,251$ & $3,004,197$ \\
\hline Captan & 5014 & Fungicide & $3,801,514$ & 585,977 \\
\hline Diuron & 1991 & Herbicide & $3,746,849$ & $3,249,062$ \\
\hline Tribufos & 8009 & Other Pesticides & $3,721,092$ & $3,978,201$ \\
\hline Acephate & 6002 & Insecticide & $3,639,113$ & $3,186,829$ \\
\hline Propargite & 6055 & Insecticide & $3,434,047$ & $1,940,567$ \\
\hline Dimethoate & 6017 & Insecticide & $3,415,912$ & $5,160,527$ \\
\hline Bromoxynil & 1116 & Herbicide & $3,379,476$ & $12,388,097$ \\
\hline Malathion & 6033 & Insecticide & $3,105,858$ & $1,935,421$ \\
\hline Ethephon & 7003 & Other Pesticides & $3,079,023$ & $2,943,740$ \\
\hline Cryolite & 6010 & Insecticide & $3,077,166$ & 479,436 \\
\hline
\end{tabular}


Table 3. Summary of national pesticide use, 1992-1995, derived from county pesticide use information sorted by total application in pounds active ingredient in descending order- Continued

\begin{tabular}{|c|c|c|c|c|}
\hline Compound & $\begin{array}{l}\text { Pesticide } \\
\text { code }\end{array}$ & Pesticide type & $\begin{array}{c}\text { Total application, (pounds, } \\
\text { active } \\
\text { ingredient) }\end{array}$ & $\begin{array}{l}\text { Total area treated } \\
\text { (acres) }\end{array}$ \\
\hline Maneb & 5009 & Fungicide & $3,008,755$ & 703,061 \\
\hline Fonofos & 6028 & Insecticide & $2,811,694$ & $2,416,793$ \\
\hline Ziram & 5016 & Fungicide & $2,760,375$ & 492,718 \\
\hline Metribuzin & 1975 & Herbicide & $2,704,051$ & $8,402,249$ \\
\hline Dimethenamid & 3001 & Herbicide & $2,615,979$ & $2,321,166$ \\
\hline Azinphos-Methyl & 6004 & Insecticide & $2,491,323$ & $2,583,532$ \\
\hline Ethalfluralin & 9009 & Herbicide & $2,459,958$ & $3,083,449$ \\
\hline Methomyl & 6038 & Insecticide & $2,449,612$ & $3,411,730$ \\
\hline Triallate & 1790 & Herbicide & $2,283,665$ & $2,224,396$ \\
\hline Norflurazon & 1018 & Herbicide & $2,271,813$ & $2,396,335$ \\
\hline Maleic Hydrazide & 8010 & Other Pesticides & $2,125,576$ & 721,431 \\
\hline Profenofos & 6084 & Insecticide & $2,121,560$ & $1,695,563$ \\
\hline Endosulfan & 6019 & Insecticide & $2,087,805$ & $1,614,778$ \\
\hline Ethyl Parathion & 6024 & Insecticide & $2,063,014$ & $3,410,741$ \\
\hline Linuron & 1993 & Herbicide & $1,974,832$ & $3,149,483$ \\
\hline Clomazone & 9001 & Herbicide & $1,961,420$ & $3,480,831$ \\
\hline Prometryn & 1987 & Herbicide & $1,845,301$ & $2,559,043$ \\
\hline Disulfoton & 6018 & Insecticide & $1,800,145$ & $2,356,733$ \\
\hline Picloram & 1051 & Herbicide & $1,723,161$ & $7,103,643$ \\
\hline PCNB & 5021 & Fungicide & $1,694,994$ & $1,586,450$ \\
\hline Acifluorfen & 1002 & Herbicide & $1,691,552$ & $7,259,825$ \\
\hline Diazinon & 6014 & Insecticide & $1,615,931$ & $1,075,288$ \\
\hline Thiodicarb & 6061 & Insecticide & $1,488,074$ & $1,964,188$ \\
\hline Thiobencarb & 1903 & Herbicide & $1,436,364$ & 473,210 \\
\hline DSMA & 4001 & Herbicide & $1,395,348$ & 767,261 \\
\hline Imazethapyr & 9000 & Herbicide & $1,377,335$ & $22,759,097$ \\
\hline Sethoxydim & 1910 & Herbicide & $1,301,674$ & $6,299,515$ \\
\hline Ethoprop & 6023 & Insecticide & $1,269,562$ & 336,202 \\
\hline Benomyl & 5001 & Fungicide & $1,250,821$ & $1,478,646$ \\
\hline Chloramben & 1299 & Herbicide & $1,216,306$ & 729,772 \\
\hline Bromacil & 1809 & Herbicide & $1,194,805$ & 440,170 \\
\hline Permethrin & 6048 & Insecticide & $1,176,636$ & $7,721,364$ \\
\hline Diclofop & 1005 & Herbicide & $1,117,669$ & $1,407,416$ \\
\hline Methamidophos & 6036 & Insecticide & $1,114,767$ & 906,920 \\
\hline Dicofol & 6016 & Insecticide & $1,090,423$ & 836,413 \\
\hline Iprodione & 5006 & Fungicide & $1,003,982$ & $1,088,045$ \\
\hline DCPA & 1872 & Herbicide & 997,630 & 184,780 \\
\hline $2,4-\mathrm{Db}$ & 1308 & Herbicide & 956,997 & $4,422,924$ \\
\hline Phosmet & 6051 & Insecticide & 929,361 & 439,807 \\
\hline Cycloate & 2069 & Herbicide & 925,958 & 363,454 \\
\hline Metiram & 5029 & Fungicide & 922,144 & 127,121 \\
\hline Metalaxyl & 5002 & Fungicide & 886,183 & $2,447,054$ \\
\hline Ethion & 6022 & Insecticide & 868,217 & 249,148 \\
\hline Sulprofos & 6059 & Insecticide & 842,478 & 530,154 \\
\hline Oxamyl & 6045 & Insecticide & 813,832 & $1,686,855$ \\
\hline Imazaquin & 4005 & Herbicide & 807,270 & $8,634,943$ \\
\hline Oryzalin & 1873 & Herbicide & 780,971 & 481,596 \\
\hline Fluazifop & 9007 & Herbicide & 755,073 & $6,809,168$ \\
\hline
\end{tabular}


Table 3. Summary of national pesticide use, 1992-1995, derived from county pesticide use information sorted by total application in pounds active ingredient in descending order- Continued

\begin{tabular}{|c|c|c|c|c|}
\hline Compound & $\begin{array}{l}\text { Pesticide } \\
\text { code }\end{array}$ & Pesticide type & $\begin{array}{c}\text { Total application, (pounds, } \\
\text { active } \\
\text { ingredient) }\end{array}$ & $\begin{array}{c}\text { Total area treated } \\
\text { (acres) }\end{array}$ \\
\hline Dicrotophos & 6082 & Insecticide & 734,486 & $2,241,589$ \\
\hline Asulam & 9048 & Herbicide & 721,007 & 334,170 \\
\hline Fenamiphos & 6025 & Insecticide & 663,777 & 481,467 \\
\hline Pebulate & 1419 & Herbicide & 560,473 & 157,205 \\
\hline Fenoxaprop & 9003 & Herbicide & 543,638 & $5,462,223$ \\
\hline Fomesafen & 4010 & Herbicide & 542,922 & $1,979,632$ \\
\hline Methidathion & 6037 & Insecticide & 520,676 & 381,805 \\
\hline Thiophanate-methyl & 5019 & Fungicide & 506,706 & 587,687 \\
\hline Ametryn & 1982 & Herbicide & 503,952 & 678,342 \\
\hline Vernolate & 1432 & Herbicide & 498,688 & 257,728 \\
\hline Methazole & 9096 & Herbicide & 491,795 & 859,590 \\
\hline Oxyfluorfen & 4000 & Herbicide & 490,752 & $1,307,624$ \\
\hline Fosetyl Al & 5031 & Fungicide & 487,594 & 148,630 \\
\hline Napropamide & 1900 & Herbicide & 473,962 & 357,759 \\
\hline Hexazinone & 2070 & Herbicide & 461,483 & 582,477 \\
\hline Thidiazuron & 8006 & Other Pesticides & 452,393 & $2,717,045$ \\
\hline Imazamethabenz & 7001 & Herbicide & 449,862 & $1,457,048$ \\
\hline Tefluthrin & 6066 & Insecticide & 423,973 & $3,562,200$ \\
\hline Benefin & 1362 & Herbicide & 416,580 & 294,474 \\
\hline Triphenyltin hydroxide & 5012 & Fungicide & 409,950 & 875,455 \\
\hline Diethatyl ethyl & 9015 & Herbicide & 397,362 & 267,249 \\
\hline Ethofumesate & 9012 & Herbicide & 384,550 & 445,594 \\
\hline Fenbutatin oxide & 6026 & Insecticide & 384,456 & 431,386 \\
\hline Lactofen & 4009 & Herbicide & 377,416 & $3,657,316$ \\
\hline Formetanate $\mathrm{HCl}$ & 6071 & Insecticide & 328,109 & 274,447 \\
\hline Bensulide & 1098 & Herbicide & 322,016 & 92,313 \\
\hline Pyrazon & 2250 & Herbicide & 321,224 & 210,153 \\
\hline Myclobutanil & 5036 & Fungicide & 317,812 & $1,166,729$ \\
\hline Dodine & 5033 & Fungicide & 297,414 & 180,706 \\
\hline Naled & 6044 & Insecticide & 295,660 & 315,531 \\
\hline Propiconazole & 5020 & Fungicide & 291,341 & $1,843,809$ \\
\hline Terbacil & 1109 & Herbicide & 284,737 & 336,750 \\
\hline Nicosulfuron & 7007 & Herbicide & 284,159 & $9,821,302$ \\
\hline Esfenvalerate & 6020 & Insecticide & 269,472 & $4,092,634$ \\
\hline Thiram & 5022 & Fungicide & 266,376 & 46,477 \\
\hline Tridiphane & 4007 & Herbicide & 255,871 & 440,172 \\
\hline Oxydemeton-methyl & 6046 & Insecticide & 242,019 & 441,160 \\
\hline Diquat & 1950 & Herbicide & 229,777 & 466,793 \\
\hline Clethodim & 7010 & Herbicide & 228,267 & $2,366,808$ \\
\hline Ferbam & 5017 & Fungicide & 223,973 & 70,174 \\
\hline Mevinphos & 6043 & Insecticide & 220,870 & 291,532 \\
\hline Cypermethrin & 6011 & Insecticide & 215,165 & $1,911,495$ \\
\hline Lambdacyhalothrin & 6083 & Insecticide & 213,447 & $2,680,334$ \\
\hline Pronamide & 1888 & Herbicide & 202,662 & 227,715 \\
\hline Difenzoquat & 1374 & Herbicide & 194,960 & 199,187 \\
\hline Amitraz & 6091 & Insecticide & 193,196 & 422,615 \\
\hline Triclopyr & 1988 & Herbicide & 192,705 & 454,123 \\
\hline Quizalofop & 7006 & Herbicide & 191,023 & $3,611,894$ \\
\hline
\end{tabular}


Table 3. Summary of national pesticide use, 1992-1995, derived from county pesticide use information sorted by total application in pounds active ingredient in descending order- Continued

\begin{tabular}{|c|c|c|c|c|}
\hline Compound & $\begin{array}{l}\text { Pesticide } \\
\text { code }\end{array}$ & Pesticide type & $\begin{array}{c}\text { Total application, (pounds, } \\
\text { active } \\
\text { ingredient) }\end{array}$ & $\begin{array}{l}\text { Total area treated } \\
\text { (acres) }\end{array}$ \\
\hline Chlorimuron & 4008 & Herbicide & 183,757 & $9,587,873$ \\
\hline Phenmedipham & 2220 & Herbicide & 183,167 & 953,543 \\
\hline DCNA & 5008 & Fungicide & 180,819 & 88,673 \\
\hline Thiabendazole & 5030 & Fungicide & 159,142 & 289,350 \\
\hline Desmedipham & 9014 & Herbicide & 154,719 & 965,871 \\
\hline Cyfluthrin & 6081 & Insecticide & 151,422 & $1,721,883$ \\
\hline Trimethacarb & 6300 & Insecticide & 151,150 & 151,150 \\
\hline Fenpropathrin & 7203 & Insecticide & 146,707 & 320,222 \\
\hline Oxythioquinox & 6047 & Insecticide & 144,872 & 206,381 \\
\hline Dimethipin & 7004 & Other Pesticides & 141,889 & 446,441 \\
\hline Isopropalin & 1867 & Herbicide & 139,291 & 139,275 \\
\hline Anilazine & 5018 & Fungicide & 139,115 & 67,408 \\
\hline Cacodylic acid & 8017 & Other Pesticides & 138,504 & 148,929 \\
\hline Quinclorac & 7013 & Herbicide & 126,968 & 342,392 \\
\hline Clopyralid & 4002 & Herbicide & 122,701 & 704,526 \\
\hline Vinclozolin & 5013 & Fungicide & 119,737 & 116,167 \\
\hline Bifenthrin & 6063 & Insecticide & 115,080 & $1,702,338$ \\
\hline Triadimefon & 5015 & Fungicide & 114,346 & 773,855 \\
\hline Diphenamid & 1366 & Herbicide & 111,217 & 49,769 \\
\hline Mepiquat chloride & 8007 & Other Pesticides & 110,871 & $2,854,066$ \\
\hline Streptomycin & 5037 & Fungicide & 108,478 & 185,628 \\
\hline Tebuthiuron & 1963 & Herbicide & 104,591 & 209,183 \\
\hline Triforine & 5003 & Fungicide & 103,491 & 143,465 \\
\hline Naptalam & 1307 & Herbicide & 99,462 & 43,631 \\
\hline Etridiazole & 5051 & Fungicide & 98,217 & 593,651 \\
\hline Bensulfuron & 7009 & Herbicide & 95,407 & 546,611 \\
\hline Methoxychlor & 6039 & Insecticide & 87,654 & 61,827 \\
\hline Thifensulfuron & 4004 & Herbicide & 82,980 & $11,648,716$ \\
\hline Pyridate & 7012 & Herbicide & 80,916 & 86,994 \\
\hline Flumetsulam & 3003 & Herbicide & 78,359 & $1,274,397$ \\
\hline Diflubenzuron & 6064 & Insecticide & 78,013 & 494,696 \\
\hline Endothall & 1948 & Herbicide & 73,964 & 443,182 \\
\hline Lindane & 6032 & Insecticide & 70,601 & 98,175 \\
\hline Dichlobenil & 1865 & Herbicide & 64,552 & 27,734 \\
\hline Primisulfuron & 7008 & Herbicide & 64,406 & $2,192,931$ \\
\hline Fenvalerate & 6070 & Insecticide & 63,136 & 306,946 \\
\hline Imidacloprid & 3004 & Insecticide & 60,966 & 957,724 \\
\hline Metaldehyde & 6073 & Insecticide & 56,890 & 82,160 \\
\hline Chlorsulfuron & 1913 & Herbicide & 56,116 & $5,536,530$ \\
\hline Propamocarb & 3007 & Fungicide & 54,198 & 55,337 \\
\hline Tralomethrin & 6067 & Insecticide & 53,331 & $1,313,008$ \\
\hline Fenarimol & 5032 & Fungicide & 50,843 & 725,430 \\
\hline Tribenuron & 7002 & Herbicide & 49,500 & $6,218,111$ \\
\hline Carboxin & 5026 & Fungicide & 44,161 & 37,249 \\
\hline Tebufenozide & 3005 & Insecticide & 42,104 & 290,444 \\
\hline MCPB & 1889 & Herbicide & 36,328 & 57,324 \\
\hline Metsulfuron & 4003 & Herbicide & 35,534 & $7,747,924$ \\
\hline Oxytetracycline & 5038 & Fungicide & 35,321 & 71,207 \\
\hline Gibberellic acid & 8013 & Other Pesticides & 33,770 & 567,810 \\
\hline
\end{tabular}


Table 3. Summary of national pesticide use, 1992-1995, derived from county pesticide use information sorted by total application in pounds active ingredient in descending order-Continued

\begin{tabular}{|c|c|c|c|c|}
\hline Compound & $\begin{array}{c}\text { Pesticide } \\
\text { code }\end{array}$ & Pesticide type & $\begin{array}{c}\text { Total application, (pounds, } \\
\text { active } \\
\text { ingredient) }\end{array}$ & $\begin{array}{c}\text { Total area treated } \\
\text { (acres) }\end{array}$ \\
\hline Triasulfuron & 7011 & Herbicide & 31,369 & $2,762,515$ \\
\hline МCPP & 1477 & Herbicide & 17,930 & 19,769 \\
\hline Clofentezine & 7204 & Insecticide & 16,133 & 73,450 \\
\hline Cyromazine & 6012 & Insecticide & 13,970 & 57,551 \\
\hline NAA & 8003 & Other Pesticides & 13,666 & 235,226 \\
\hline Abamectin & 6001 & Insecticide & 12,779 & $1,021,633$ \\
\hline Dinocap & 5035 & Fungicide & 11,238 & 23,252 \\
\hline Trichlorfon & 6062 & Insecticide & 10,356 & 19,607 \\
\hline Dimethomorph & 3006 & Fungicide & 6,678 & 26,656 \\
\hline NAD & 8014 & Other Pesticides & 3,974 & 51,143 \\
\hline Cytokinins & 8015 & Other Pesticides & 3,166 & 105,776 \\
\hline Cymoxanil & 3008 & Fungicide & 2,332 & 12,979 \\
\hline Siduron & 1984 & Herbicide & 247 & 62 \\
\hline $\mathrm{Bt}$ & 6005 & Insecticide & No Data & No Data \\
\hline
\end{tabular}


Table 4. Primary crops that account for use of each pesticide, 1992- 1995

$[-$, no data $]$

\begin{tabular}{|c|c|c|c|c|c|c|c|}
\hline Compound & Crop & $\begin{array}{l}\text { Total } \\
\text { amount } \\
\text { applied } \\
\text { (pounds) }\end{array}$ & $\begin{array}{l}\text { Total area } \\
\text { treated } \\
\text { (acres) }\end{array}$ & Compound & Crop & $\begin{array}{l}\text { Total } \\
\text { amount } \\
\text { applied } \\
\text { (pounds) }\end{array}$ & $\begin{array}{l}\text { Total area } \\
\text { treated } \\
\text { (acres) }\end{array}$ \\
\hline \multirow[t]{11}{*}{ Abamectin } & Cotton & 6,285 & 629,823 & \multirow[t]{2}{*}{ Ametryn } & \multirow{2}{*}{$\begin{array}{l}\text { Sugar Cane for Seed } \\
\text { and Sugar }\end{array}$} & \multirow[t]{2}{*}{75,137} & \multirow[t]{2}{*}{179,595} \\
\hline & All Citrus & 1,755 & 175,453 & & & & \\
\hline & Pears & 1,503 & 61,495 & \multirow[t]{2}{*}{ Amitraz } & Cotton & 116,056 & 392,065 \\
\hline & Hops & 873 & 33,808 & & Pears & 77,140 & 30,551 \\
\hline & Strawberries & 746 & 18,657 & \multirow{7}{*}{ Anilazine } & & & \\
\hline & Tomatoes & 580 & 19,324 & & Tomatoes & 108,832 & 56,849 \\
\hline & Lettuce & 549 & 51,903 & & Strawberries & 20,930 & 6,266 \\
\hline & Celery & 326 & 15,759 & & Celery & 7,705 & 3,044 \\
\hline & Potatoes & 105 & 10,464 & & Onions & 1,042 & 700 \\
\hline & \multirow[t]{2}{*}{ Sweet Peppers } & \multirow[t]{2}{*}{53} & \multirow[t]{2}{*}{4,533} & & Potatoes & 363 & 385 \\
\hline & & & & & Garlic & 244 & 163 \\
\hline \multirow[t]{11}{*}{ Acephate } & Tobacco & $1,672,309$ & 798,896 & \multirow{4}{*}{ Asulam } & \multirow{4}{*}{$\begin{array}{l}\text { Sugar Cane for Seed } \\
\text { and Sugar } \\
\text { Sod }\end{array}$} & \multirow[b]{2}{*}{698,293} & \multirow[b]{2}{*}{322,812} \\
\hline & Cotton & $1,369,895$ & $1,782,679$ & & & & \\
\hline & Lettuce & 123,736 & 108,418 & & & 22,715 & 11,357 \\
\hline & Soybeans & 84,504 & 90,814 & & & 22,713 & \\
\hline & Peanuts & 82,938 & 158,327 & \multirow{7}{*}{ Atrazine } & Corn & $53,796,206$ & 48.041 .955 \\
\hline & Mint & 81,949 & 72,206 & & Sorghum & $7,339,963$ & $7,385,377$ \\
\hline & Green Beans & 74,586 & 64,840 & & Sugar Cane for Seed & & 805742 \\
\hline & Sweet Peppers & 63,244 & 27,937 & & $\begin{array}{l}\text { Sugar Cane ror seed } \\
\text { and Sugar }\end{array}$ & $1, / 11,322$ & 800,142 \\
\hline & Celery & 29,751 & 20,484 & & All Pasture & 518,074 & 270,276 \\
\hline & Dry Beans & 28,266 & 32,048 & & Sweet Corn & 444,523 & 411,171 \\
\hline & \multirow{3}{*}{ Corn } & \multirow{3}{*}{$23,841,035$} & \multirow{3}{*}{$11,845,501$} & & Sod & 119,182 & 53,790 \\
\hline \multirow[t]{2}{*}{ Acetochlor } & & & & & Proso Millet & 11,937 & 11,937 \\
\hline & & & & & Field and Grass Seed & 6,305 & 5,506 \\
\hline \multirow[t]{4}{*}{ Acifluorfen } & Soybeans & $1,517,072$ & $6,779,706$ & & & & \\
\hline & Peanuts & 148,196 & 394,524 & Azinphos-methyl & Apples & 830,812 & 429,015 \\
\hline & Rice & 26,284 & 85,596 & & Cotton & 671,920 & $1,334,476$ \\
\hline & & & & & Almonds & 221,948 & \\
\hline Alachlor & Corn & $13,902,747$ & $8,114,192$ & & Sugar Cane for Seed & 179,087 & 205,797 \\
\hline & Soybeans & $8,862,899$ & $4,848,681$ & & and Sugar & & \\
\hline & Sorghum & $1,829,617$ & $1,096,950$ & & Pears & 137,429 & 64,319 \\
\hline & Sweet Corn & 507,232 & 231,950 & & Peaches & 91,708 & 52,721 \\
\hline & Dry Beans & 336,653 & 157,500 & & Potatoes & 80,724 & \\
\hline & Peanuts & 122,871 & 30,718 & & Walnuts & 56,126 & 33,811 \\
\hline & Cotton & 61,870 & 41,247 & & Pistachios & 48,269 & 22,011 \\
\hline & Sunflowers & 23,794 & 8,134 & & Cherries & 46,360 & 37,144 \\
\hline Aldicarb & Cotton & $2,294,875$ & $3,139,633$ & Benefin & Peanuts & 273,349 & 182,233 \\
\hline & Peanuts & $1,019,803$ & 751,643 & & Tobacco & 62,683 & 47,487 \\
\hline & Sugar Beets for Sugar & 398,354 & 165,125 & & Alfalfa Hay & 43,495 & 34,592 \\
\hline & All Citrus & 301,343 & 102,847 & & Lettuce & 28,089 & 22,978 \\
\hline & Tobacco & 167,788 & 74,572 & & Field and Grass Seed & 6,488 & 5,947 \\
\hline & Pecans & 42,323 & 17,227 & & Sod & 2,476 & 1,237 \\
\hline & Dry Beans & 31,481 & 21,643 & & & & \\
\hline & Sorghum & 19,351 & 40,314 & Benomyl & Grapes & 327,596 & 64,240 \\
\hline & Sweet Potatoes & 7,433 & 2,652 & & Rice & 303,966 & 572,721 \\
\hline & & & & & Wheat for Grain & 118,226 & 243,111 \\
\hline Ametryn & Corn & 428,815 & 498,747 & & Tomatoes & 57,086 & 38,592 \\
\hline
\end{tabular}


Table 4. Primary crops that account for use of each pesticide, 1992-1995- Continued

\begin{tabular}{|c|c|c|c|c|c|c|c|}
\hline Compound & Crop & $\begin{array}{c}\text { Total } \\
\text { amount } \\
\text { applied } \\
\text { (pounds) }\end{array}$ & $\begin{array}{c}\text { Total area } \\
\text { treated } \\
\text { (acres) }\end{array}$ & Compound & Crop & $\begin{array}{c}\text { Total } \\
\text { amount } \\
\text { applied } \\
\text { (pounds) }\end{array}$ & $\begin{array}{c}\text { Total area } \\
\text { treated } \\
\text { (acres) }\end{array}$ \\
\hline \multirow[t]{7}{*}{$\overline{\text { Benomyl }}$} & Green Beans & 55,404 & 54,384 & $\overline{\text { Bromoxynil }}$ & Oats & 44,287 & 103,427 \\
\hline & Apples & 51,445 & 63,030 & & Rice & 41,296 & 196,015 \\
\hline & Almonds & 50,060 & 66,129 & & Mint & 23,241 & 79,898 \\
\hline & Peaches & 34,536 & 40,167 & & Onions & 14,026 & 56,303 \\
\hline & Pecans & 31,252 & 30,549 & & Garlic & 13,893 & 17,380 \\
\hline & Watermelons & 30,024 & 44,510 & & & & \\
\hline & & & & $\mathrm{Bt}$ & Alfalfa Hay & - & 50,247 \\
\hline \multirow[t]{2}{*}{ Bensulfuron } & Rice & 95,407 & 546,611 & & Almonds & - & 114,624 \\
\hline & & & & & Apples & - & 53,138 \\
\hline \multirow[t]{10}{*}{ Bensulide } & Onions & 58,658 & 14,200 & & Apricots & - & 2,150 \\
\hline & Cucumbers & 53,194 & 15,419 & & Artichokes & - & 3,154 \\
\hline & Sod & 42,482 & 6,461 & & Asparagus & - & 1,043 \\
\hline & Watermelons & 29,436 & 8,987 & & Avocados & - & 671 \\
\hline & Lettuce & 28,134 & 7,192 & & Beets & - & 196 \\
\hline & Cantaloupes & 26,883 & 9,824 & & Sugar Beets for Sugar & - & 3,427 \\
\hline & Squash & 20,951 & 8,314 & & Blackberries & - & 1,055 \\
\hline & Pumpkins & 20,781 & 8,932 & & & & \\
\hline & Carrots & 8,451 & 1,761 & Butylate & Corn & $7,648,802$ & $1,973,720$ \\
\hline & Hot Peppers & 8,231 & 2,993 & & Sweet Corn & 52,692 & 12,434 \\
\hline \multirow[t]{11}{*}{ Bentazon } & Soybeans & $4,828,007$ & $7,084,094$ & Cacodylic acid & Cotton & 138,504 & 148,929 \\
\hline & Peanuts & 767,893 & $1,023,858$ & & & & \\
\hline & Corn & 564,829 & $1,231,725$ & Captan & Apples & $2,264,488$ & 257,199 \\
\hline & Mint & 166,465 & 111,793 & & Peaches & 540,337 & 83,938 \\
\hline & Dry Beans & 153,836 & 201,866 & & Strawberries & 275,545 & 23,266 \\
\hline & Rice & 101,933 & 156,492 & & Almonds & 187,984 & 57,312 \\
\hline & Sweet Corn & 59,169 & 109,705 & & Blueberries & 143,439 & 35,228 \\
\hline & Green Peas & 49,936 & 59,269 & & Plums & 123,858 & 39,590 \\
\hline & Green Beans & 27,255 & 32,292 & & Grapes & 115,746 & 53,646 \\
\hline & Sorghum & 5,739 & 5,739 & & Cherries & 86,758 & 15,413 \\
\hline & & & & & Raspberries & 23,563 & 5,636 \\
\hline \multirow[t]{11}{*}{ Bifenthrin } & Cotton & 70,171 & 966,149 & & Pumpkins & 12,949 & 1,816 \\
\hline & Corn & 21,728 & 467,423 & & & & \\
\hline & Alfalfa Hay & 14,518 & 177,320 & Carbaryl & Alfalfa Hay & 490,173 & 320,562 \\
\hline & Hops & 2,910 & 27,059 & & Corn & 427,446 & 337,061 \\
\hline & Cantaloupes & 1,573 & 17,478 & & Pecans & 423,525 & 99,815 \\
\hline & Field and Grass Seed & 1,377 & 17,217 & & Other Hay & 358,777 & 358,777 \\
\hline & Melons & 743 & 7,434 & & All Citrus & 339,934 & 42,039 \\
\hline & Watermelons & 686 & 5,941 & & Soybeans & 336,308 & 389,564 \\
\hline & Raspberries & 407 & 4,069 & & Apples & 281,131 & 192,238 \\
\hline & Squash & 264 & 2,939 & & Sorghum & 260,043 & 191,714 \\
\hline & & & & & Grapes & 200,728 & 80,956 \\
\hline Bromacil & All Citrus & $1,194,805$ & 440,170 & & Peaches & 146,008 & 40,335 \\
\hline \multirow[t]{5}{*}{ Bromoxynil } & Corn & $1,528,361$ & $6,261,478$ & Carbofuran & Corn & $2,480,218$ & $2,586,374$ \\
\hline & Wheat for Grain & $1,273,103$ & $4,426,559$ & & Alfalfa Hay & 999,611 & $2,027,644$ \\
\hline & Barley & 262,377 & 766,415 & & Sorghum & 389,221 & 440,092 \\
\hline & Alfalfa Hay & 81,205 & 156,715 & & Potatoes & 297,440 & 245,107 \\
\hline & Sorghum & 79,103 & 273,845 & & Rice & 206,884 & 410,101 \\
\hline
\end{tabular}


Table 4. Primary crops that account for use of each pesticide, 1992-1995- Continued

\begin{tabular}{|c|c|c|c|c|c|c|c|}
\hline Compound & Crop & $\begin{array}{c}\text { Total } \\
\text { amount } \\
\text { applied } \\
\text { (pounds) }\end{array}$ & $\begin{array}{c}\text { Total area } \\
\text { treated } \\
\text { (acres) }\end{array}$ & Compound & Crop & $\begin{array}{c}\text { Total } \\
\text { amount } \\
\text { applied } \\
\text { (pounds) }\end{array}$ & $\begin{array}{c}\text { Total area } \\
\text { treated } \\
\text { (acres) }\end{array}$ \\
\hline \multirow[t]{6}{*}{$\overline{\text { Carbofuran }}$} & Cotton & 172,838 & 472,519 & \multirow[t]{6}{*}{ Chlorpyrifos } & Wheat for Grain & 741,185 & $1,519,246$ \\
\hline & Tobacco & 165,462 & 46,450 & & Tobacco & 708,200 & 300,604 \\
\hline & Grapes & 120,192 & 29,211 & & All Citrus & 675,080 & 238,426 \\
\hline & \multirow{2}{*}{$\begin{array}{l}\text { Sugar Cane for Seed } \\
\text { and Sugar }\end{array}$} & \multirow[t]{2}{*}{87,499} & \multirow[t]{2}{*}{52,861} & & Apples & 654,938 & 333,527 \\
\hline & & & & & \multirow{2}{*}{$\begin{array}{l}\text { Sugar Beets for Sugar } \\
\text { Pecans }\end{array}$} & 417,423 & \multirow{2}{*}{$\begin{array}{l}310,460 \\
169,144\end{array}$} \\
\hline & Sugar Beets for Sugar & 77,952 & 60,260 & & & 330,969 & \\
\hline \multirow[t]{2}{*}{ Carboxin } & Peanuts & 44,161 & 37,249 & \multirow[t]{4}{*}{ Chlorsulfuron } & Wheat for Grain & 55,302 & $5,480,806$ \\
\hline & & & & & Barley & 658 & 41,598 \\
\hline \multirow[t]{10}{*}{ Chloramben } & Dry Beans & 582,017 & 290,565 & & Oats & 156 & 14,125 \\
\hline & Soybeans & 456,076 & 367,464 & & & & \\
\hline & Squash & 45,095 & 16,613 & \multirow{2}{*}{ Clethodim } & \multirow{2}{*}{ Soybeans } & \multirow{2}{*}{228,267} & \multirow{2}{*}{$2,366,808$} \\
\hline & Pumpkins & 36,031 & 13,703 & & & & \\
\hline & Sunflowers & 33,813 & 11,271 & \multirow[t]{6}{*}{ Clofentezine } & Walnuts & 9,911 & 14,792 \\
\hline & Sweet Potatoes & 24,268 & 7,823 & & Almonds & 3,968 & 39,678 \\
\hline & Green Beans & 19,742 & 12,503 & & Pears & 1,220 & 8,070 \\
\hline & Tomatoes & 11,769 & 5,073 & & Peaches & 508 & 5,648 \\
\hline & Cucumbers & 4,852 & 3,849 & & Nectarines & 275 & 2,503 \\
\hline & Sweet Peppers & 2,618 & 873 & & Cherries & 251 & 2,758 \\
\hline \multirow[t]{3}{*}{ Chlorimuron } & Soybeans & 180,752 & $9,212,227$ & \multirow[t]{9}{*}{ Clomazone } & Soybeans & $1,306,459$ & $2,249,124$ \\
\hline & Peanuts & 3,005 & 375,646 & & Cotton & 625,858 & $1,177,648$ \\
\hline & & & & & Sweet Potatoes & 15,191 & 20,255 \\
\hline \multirow[t]{11}{*}{ Chloropicrin } & Tomatoes & $3,021,015$ & 50,803 & & Green Peas & 9,973 & 26,020 \\
\hline & Strawberries & $2,332,362$ & 25,925 & & Pumpkins & 1,430 & 2,033 \\
\hline & Potatoes & 657,389 & 13,598 & & Squash & 1,052 & 1,237 \\
\hline & Tobacco & 603,745 & 12,534 & & Sweet Peppers & 744 & 1,786 \\
\hline & Sweet Peppers & 533,555 & 6,604 & & Cabbage & 713 & 2,728 \\
\hline & Onions & 107,425 & 3,548 & & & & \\
\hline & Lettuce & 76,338 & 1,957 & \multirow[t]{8}{*}{ Clopyralid } & Wheat for Grain & 50,301 & 436,214 \\
\hline & Eggplant & 56,895 & 902 & & Sugar Beets for Sugar & 36,891 & 145,797 \\
\hline & Green Onions & 22,681 & 418 & & Mint & 27,024 & 46,650 \\
\hline & Hot Peppers & 9,543 & 187 & & Oats & 3,892 & 38,920 \\
\hline & & & & & Barley & 3,435 & 28,628 \\
\hline \multirow[t]{11}{*}{ Chlorothalonil } & Peanuts & $5,926,168$ & $1,362,273$ & & Field and Grass Seed & 1,120 & 7,997 \\
\hline & Potatoes & $2,790,349$ & 806,827 & & Canola & 38 & 320 \\
\hline & Tomatoes & $1,230,116$ & 191,811 & & & & \\
\hline & Watermelons & 423,300 & 91,746 & \multirow[t]{11}{*}{ Copper } & All Citrus & $2,061,386$ & 477,675 \\
\hline & Onions & 294,317 & 80,175 & & Rice & $1,870,820$ & 167,520 \\
\hline & Squash & 257,604 & 32,805 & & Tomatoes & $1,149,677$ & 165,806 \\
\hline & Cucumbers & 239,026 & 44,338 & & Walnuts & $1,145,976$ & 212,840 \\
\hline & Peaches & 213,755 & 60,553 & & Almonds & $1,000,521$ & 233,657 \\
\hline & Celery & 208,396 & 28,783 & & Peaches & 590,181 & 84,367 \\
\hline & Cherries & 149,219 & 33,165 & & Grapes & 393,232 & 219,520 \\
\hline & & & & & Potatoes & 323,176 & 286,093 \\
\hline Chlorpyrifos & Corn & $6,710,506$ & $6,253,026$ & & Nectarines & 241,373 & 35,759 \\
\hline & Cotton & $1,616,065$ & $1,394,490$ & & Apples & 211,019 & 69,700 \\
\hline & Peanuts & 912,717 & 583,139 & & & & \\
\hline & Alfalfa Hay & 872,215 & $1,273,962$ & Cryolite & Grapes & $2,552,815$ & 437,126 \\
\hline
\end{tabular}


Table 4. Primary crops that account for use of each pesticide, 1992-1995- Continued

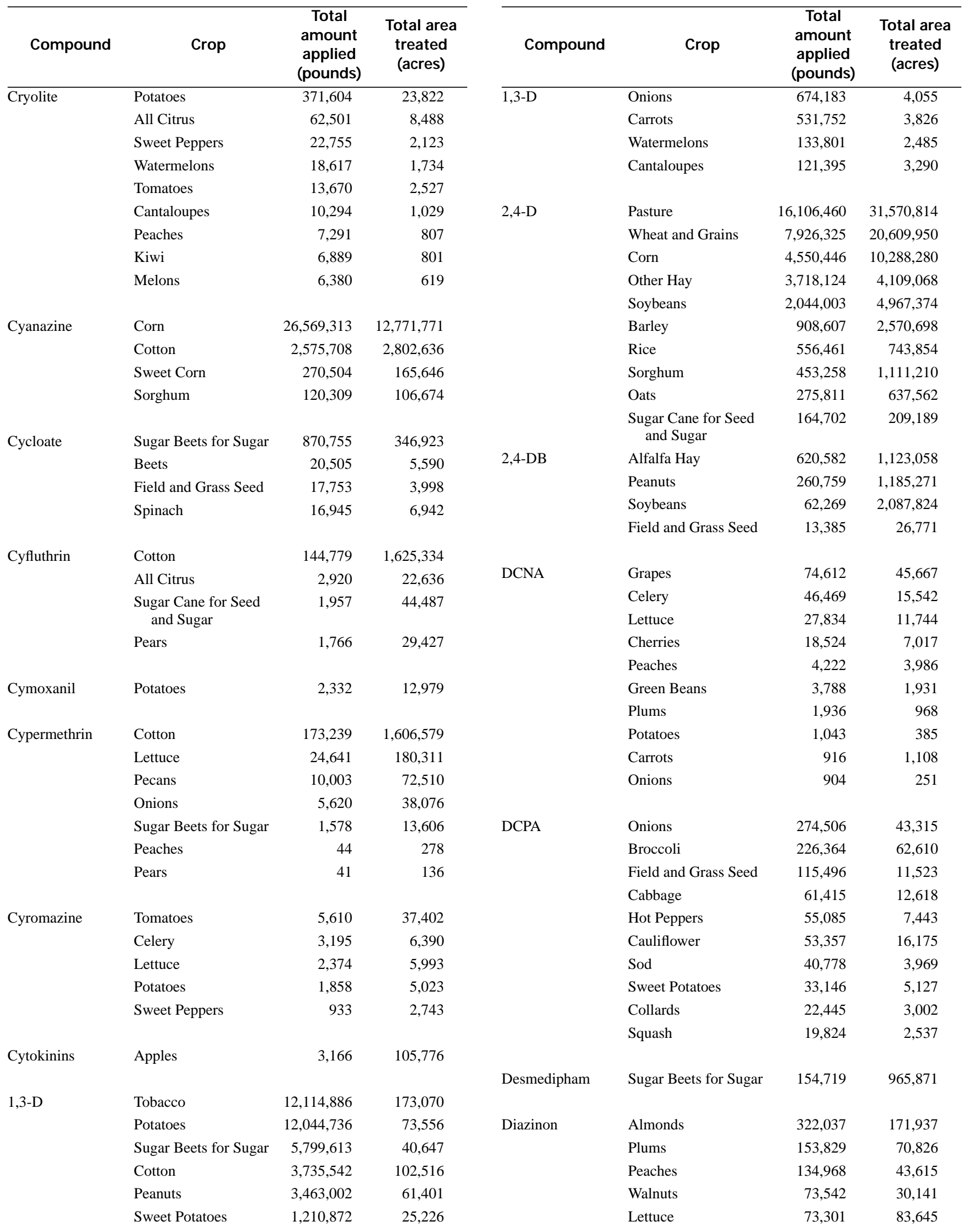


Table 4. Primary crops that account for use of each pesticide, 1992-1995- Continued

\begin{tabular}{|c|c|c|c|c|c|c|c|}
\hline Compound & Crop & $\begin{array}{l}\text { Total } \\
\text { amount } \\
\text { applied } \\
\text { (pounds) }\end{array}$ & $\begin{array}{c}\text { Total area } \\
\text { treated } \\
\text { (acres) }\end{array}$ & Compound & Crop & $\begin{array}{c}\text { Total } \\
\text { amount } \\
\text { applied } \\
\text { (pounds) }\end{array}$ & $\begin{array}{c}\text { Total area } \\
\text { treated } \\
\text { (acres) }\end{array}$ \\
\hline \multirow[t]{6}{*}{ Diazinon } & Nectarines & 67,184 & 27,534 & $\overline{\text { Difenzoquat }}$ & Barley & 69,737 & 77,886 \\
\hline & Sweet Corn & 67,027 & 27,614 & & & & \\
\hline & Tobacco & 55,619 & 22,141 & Diflubenzuron & Cotton & 78,013 & 494,696 \\
\hline & Apples & 52,499 & 29,885 & & & & \\
\hline & All Citrus & 46,215 & 23,284 & Dimethenamid & Corn & $2,354,305$ & $2,056,849$ \\
\hline & & & & & Soybeans & 261,674 & 264,317 \\
\hline \multirow[t]{11}{*}{ Dicamba } & Corn & $6,907,941$ & $19,467,522$ & & & & \\
\hline & All Pasture & $1,175,374$ & $6,764,796$ & Dimethipin & Cotton & 141,889 & 446,441 \\
\hline & Wheat for Grain & 861,932 & $10,228,079$ & & & & \\
\hline & Other Hay & 398,438 & $1,220,359$ & Dimethoate & Wheat for Grain & $1,269,355$ & 967,133 \\
\hline & Barley & 89,182 & 501,438 & & Alfalfa Hay & 467,016 & 942,931 \\
\hline & Sorghum & 59,065 & 182,481 & & Cotton & 315,691 & $1,121,223$ \\
\hline & Field and Grass Seed & 50,572 & 300,654 & & Corn & 233,048 & 498,141 \\
\hline & Oats & 32,167 & 244,093 & & Sorghum & 213,864 & 509,464 \\
\hline & Sod & 11,519 & 32,349 & & All Citrus & 210,037 & 117,317 \\
\hline & Proso Millet & 1,726 & 15,229 & & Apples & 107,920 & 87,178 \\
\hline & & & & & Grapes & 105,090 & 61,818 \\
\hline \multirow[t]{11}{*}{ Dichlobenil } & Cranberries & 38,934 & 16,102 & & Pecans & 72,085 & 68,766 \\
\hline & Apples & 10,705 & 5,352 & & Lettuce & 57,122 & 130,339 \\
\hline & Blueberries & 6,246 & 3,174 & & & & \\
\hline & Filberts & 3,858 & 1,286 & Dimethomorph & Potatoes & 6,678 & 26,656 \\
\hline & Pears & 1,580 & 632 & & & & \\
\hline & Cherries & 1,236 & 412 & Dinocap & Apples & 5,693 & 6,721 \\
\hline & Peaches & 769 & 269 & & Grapes & 4,534 & 14,666 \\
\hline & Raspberries & 554 & 222 & & Pears & 818 & 1,544 \\
\hline & Plums & 504 & 202 & & Watermelons & 193 & 322 \\
\hline & Blackberries & 166 & 83 & & & & \\
\hline & & & & Diphenamid & Tobacco & 87,941 & 43,970 \\
\hline \multirow[t]{3}{*}{ Diclofop } & Wheat for Grain & $1,043,366$ & $1,309,412$ & & Sweet Potatoes & 15,711 & 3,928 \\
\hline & Barley & 74,303 & 98,004 & & Strawberries & 7,034 & 1,484 \\
\hline & & & & & Sweet Peppers & 531 & 386 \\
\hline \multirow[t]{11}{*}{ Dicofol } & Cotton & 527,705 & 474,849 & & & & \\
\hline & All Citrus & 302,464 & 133,593 & Diquat & Potatoes & 176,943 & 378,942 \\
\hline & Dry Beans & 109,230 & 102,863 & & Alfalfa Hay & 49,956 & 83,284 \\
\hline & Apples & 48,079 & 28,363 & & Field and Grass Seed & 2,878 & 4,568 \\
\hline & Grapes & 22,230 & 22,212 & & & & \\
\hline & Pecans & 17,456 & 8,728 & Disulfoton & Cotton & 495,256 & 954,116 \\
\hline & Mint & 11,394 & 9,495 & & Wheat for Grain & 475,657 & 663,295 \\
\hline & Tomatoes & 11,324 & 10,257 & & Corn & 203,834 & 204,357 \\
\hline & Strawberries & 10,315 & 5,765 & & Potatoes & 184,498 & 63,529 \\
\hline & Watermelons & 8,362 & 10,939 & & Peanuts & 113,906 & 111,641 \\
\hline & & & & & Sorghum & 92,029 & 188,038 \\
\hline \multirow[t]{2}{*}{ Dicrotophos } & Cotton & 734,486 & $2,241,589$ & & Tobacco & 54,341 & 13,662 \\
\hline & & & & & Asparagus & 38,770 & 30,010 \\
\hline \multirow[t]{4}{*}{ Diethatyl ethyl } & Sugar Beets for Sugar & 380,017 & 260,441 & & Barley & 22,533 & 27,800 \\
\hline & Spinach & 16,552 & 6,435 & & Lettuce & 16,479 & 11,743 \\
\hline & Beets & 793 & 373 & & & & \\
\hline & & & & Diuron & All Citrus & $1,086,503$ & 531,931 \\
\hline Difenzoquat & Wheat for Grain & 125,223 & 121,301 & & Wheat for Grain & 777,808 & 635,746 \\
\hline
\end{tabular}


Table 4. Primary crops that account for use of each pesticide, 1992-1995- Continued

\begin{tabular}{|c|c|c|c|c|c|c|c|}
\hline Compound & Crop & $\begin{array}{c}\text { Total } \\
\text { amount } \\
\text { applied } \\
\text { (pounds) }\end{array}$ & $\begin{array}{c}\text { Total area } \\
\text { treated } \\
\text { (acres) }\end{array}$ & Compound & Crop & $\begin{array}{c}\text { Total } \\
\text { amount } \\
\text { applied } \\
\text { (pounds) }\end{array}$ & $\begin{array}{c}\text { Total area } \\
\text { treated } \\
\text { (acres) }\end{array}$ \\
\hline \multirow[t]{8}{*}{ Diuron } & Cotton & 742,308 & $1,170,832$ & Esfenvalerate & Tomatoes & 22,379 & 196,774 \\
\hline & Field and Grass Seed & 438,267 & 339,373 & & Soybeans & 12,969 & 154,610 \\
\hline & Alfalfa Hay & 208,984 & 148,015 & & Pecans & 11,889 & 80,501 \\
\hline & Grapes & 101,155 & 102,603 & & Potatoes & 11,838 & 225,788 \\
\hline & Other Hay & 74,096 & 74,096 & & Sweet Corn & 9,633 & 83,819 \\
\hline & Asparagus & 64,429 & 43,588 & & Wheat for Grain & 5,862 & 195,392 \\
\hline & Apples & 64,273 & 56,512 & & Cabbage & 5,618 & 32,357 \\
\hline & Walnuts & 33,017 & 25,358 & & Sunflowers & 5,342 & 173,127 \\
\hline \multirow[t]{7}{*}{ Dodine } & Apples & 179,692 & 100,438 & Ethalfluralin & Soybeans & $1,061,584$ & $1,294,110$ \\
\hline & Cherries & 40,805 & 25,371 & & Peanuts & 466,214 & 625,728 \\
\hline & Pears & 31,784 & 17,966 & & Dry Beans & 456,544 & 536,465 \\
\hline & Pecans & 31,130 & 30,822 & & Sunflowers & 399,178 & 530,636 \\
\hline & Peaches & 13,858 & 5,965 & & Cucumbers & 34,360 & 45,149 \\
\hline & Strawberries & 144 & 144 & & Watermelons & 18,404 & 25,198 \\
\hline & & & & & Green Beans & 8,770 & 7,358 \\
\hline \multirow[t]{3}{*}{ DSMA } & Cotton & $1,394,496$ & 766,409 & & Cantaloupes & 6,405 & 8,010 \\
\hline & Sod & 852 & 852 & & Dry Peas & 3,922 & 6,878 \\
\hline & & & & & Squash & 2,927 & 2,090 \\
\hline \multirow[t]{11}{*}{ Endosulfan } & Cotton & 736,166 & 742,134 & & & & \\
\hline & Apples & 229,888 & 138,716 & Ethephon & Cotton & $2,869,000$ & $2,662,406$ \\
\hline & Tomatoes & 225,505 & 71,494 & & Tobacco & 121,967 & 101,639 \\
\hline & Potatoes & 195,041 & 195,208 & & Tomatoes & 39,378 & 70,725 \\
\hline & Pecans & 130,538 & 80,216 & & Apples & 20,445 & 30,618 \\
\hline & Pears & 74,803 & 32,585 & & Walnuts & 11,399 & 12,679 \\
\hline & Lettuce & 61,006 & 49,737 & & Grapes & 8,125 & 30,467 \\
\hline & Grapes & 56,636 & 41,681 & & Cherries & 4,620 & 24,204 \\
\hline & Squash & 42,476 & 20,741 & & Hot Peppers & 2,129 & 3,548 \\
\hline & Peaches & 39,831 & 27,497 & & Barley & 944 & 2,008 \\
\hline & & & & & Sweet Peppers & 442 & 579 \\
\hline \multirow[t]{6}{*}{ Endothall } & Cotton & 28,397 & 372,014 & & & & \\
\hline & Potatoes & 22,616 & 24,955 & Ethion & All Citrus & 841,628 & 225,953 \\
\hline & Sugar Beets for Sugar & 10,647 & 32,002 & & Onions & 8,918 & 8,918 \\
\hline & Field and Grass Seed & 10,045 & 9,695 & & Pecans & 6,720 & 8,728 \\
\hline & Hops & 2,258 & 4,516 & & Apples & 5,838 & 3,970 \\
\hline & & & & & Avocados & 4,691 & 1,167 \\
\hline \multirow[t]{11}{*}{ EPTC } & Corn & $9,102,979$ & $2,330,408$ & & Melons & 423 & 413 \\
\hline & Dry Beans & $1,403,879$ & 501,752 & & & & \\
\hline & Potatoes & $1,313,936$ & 373,974 & Ethofumesate & Sugar Beets for Sugar & 342,531 & 325,583 \\
\hline & Alfalfa Hay & $1,161,549$ & 406,879 & & Field and Grass Seed & 41,984 & 119,955 \\
\hline & Sugar Beets for Sugar & 425,001 & 171,044 & & Beets & 34 & 56 \\
\hline & Green Beans & 300,475 & 112,760 & & & & \\
\hline & Sweet Corn & 220,464 & 47,104 & Ethoprop & Potatoes & 495,748 & 114,710 \\
\hline & Tomatoes & 50,942 & 20,215 & & Tobacco & 426,341 & 102,581 \\
\hline & Field and Grass Seed & 37,034 & 14,081 & & Sugar Cane for Seed & 181,703 & 39,346 \\
\hline & Safflower & 35,000 & 10,086 & & and Sugar & & \\
\hline & & & & & Peanuts & 48,387 & 24,193 \\
\hline \multirow[t]{2}{*}{ Esfenvalerate } & Cotton & 99,096 & $1,119,890$ & & Sweet Potatoes & 41,409 & 8,826 \\
\hline & Corn & 31,393 & 759,149 & & Sweet Corn & 36,047 & 26,826 \\
\hline
\end{tabular}


Table 4. Primary crops that account for use of each pesticide, 1992-1995- Continued

\begin{tabular}{|c|c|c|c|c|c|c|c|}
\hline Compound & Crop & $\begin{array}{c}\text { Total } \\
\text { amount } \\
\text { applied } \\
\text { (pounds) }\end{array}$ & $\begin{array}{c}\text { Total area } \\
\text { treated } \\
\text { (acres) }\end{array}$ & Compound & Crop & $\begin{array}{c}\text { Total } \\
\text { amount } \\
\text { applied } \\
\text { (pounds) }\end{array}$ & $\begin{array}{c}\text { Total area } \\
\text { treated } \\
\text { (acres) }\end{array}$ \\
\hline \multirow[t]{5}{*}{ Ethoprop } & Green Beans & 22,392 & 12,046 & Fenpropathrin & Cotton & 135,514 & 282,322 \\
\hline & Corn & 13,971 & 5,566 & & All Citrus & 6,144 & 19,199 \\
\hline & Cabbage & 1,970 & 655 & & Tomatoes & 5,049 & 18,701 \\
\hline & Cucumbers & 1,594 & 1,452 & & & & \\
\hline & & & & Fenvalerate & Cotton & 26,116 & 105,879 \\
\hline \multirow[t]{11}{*}{ Ethyl parathion } & Sorghum & 397,593 & 696,033 & & Corn & 16,132 & 87,015 \\
\hline & Corn & 376,784 & 432,250 & & Potatoes & 4,790 & 24,731 \\
\hline & Alfalfa Hay & 370,659 & 683,717 & & Soybeans & 4,548 & 22,739 \\
\hline & Wheat for Grain & 338,630 & 782,130 & & Sorghum & 4,031 & 40,314 \\
\hline & Sunflowers & 240,192 & 216,712 & & Squash & 2,299 & 3,886 \\
\hline & Cotton & 206,221 & 331,126 & & Alfalfa Hay & 1,578 & 7,890 \\
\hline & Soybeans & 109,005 & 218,009 & & Apples & 906 & 1,132 \\
\hline & Barley & 19,356 & 41,299 & & Green Beans & 598 & 3,224 \\
\hline & Oats & 3,460 & 7,053 & & Sunflowers & 584 & 3,896 \\
\hline & Rye & 1,085 & 2,170 & & & & \\
\hline & & & & Ferbam & Grapes & 74,813 & 25,533 \\
\hline \multirow[t]{2}{*}{ Etridiazole } & Cotton & 98,217 & 593,651 & & Peaches & 73,804 & 28,086 \\
\hline & & & & & Cherries & 34,427 & 7,460 \\
\hline \multirow[t]{11}{*}{ Fenamiphos } & Tobacco & 271,813 & 241,723 & & Cranberries & 20,600 & 4,601 \\
\hline & Grapes & 148,506 & 101,996 & & Apples & 10,558 & 1,957 \\
\hline & Cotton & 121,642 & 82,501 & & Pears & 8,502 & 1,703 \\
\hline & Peanuts & 47,266 & 18,782 & & Raspberries & 1,269 & 835 \\
\hline & Broccoli & 32,203 & 16,514 & & & & \\
\hline & All Citrus & 16,807 & 8,488 & Fluazifop & Soybeans & 569,653 & $5,770,351$ \\
\hline & Cauliflower & 9,596 & 5,515 & & Cotton & 160,368 & 912,188 \\
\hline & Peaches & 4,873 & 1,418 & & Onions & 7,377 & 30,225 \\
\hline & Cherries & 2,570 & 487 & & All Citrus & 4,756 & 15,341 \\
\hline & Cabbage & 2,559 & 1,738 & & Sweet Potatoes & 3,523 & 33,243 \\
\hline & & & & & Carrots & 3,342 & 17,582 \\
\hline \multirow[t]{4}{*}{ Fenarimol } & Apples & 26,437 & 190,047 & & Peanuts & 3,123 & 16,436 \\
\hline & Grapes & 20,941 & 496,955 & & Mint & 1,085 & 6,825 \\
\hline & Cherries & 2,526 & 26,757 & & Garlic & 953 & 3,300 \\
\hline & Pears & 939 & 11,670 & & Lettuce & 742 & 2,852 \\
\hline \multirow[t]{11}{*}{ Fenbutatin oxide } & All Citrus & 223,611 & 224,188 & Flumetsulam & Corn & 41,443 & 665,461 \\
\hline & Pecans & 53,835 & 53,835 & & Soybeans & 36,916 & 608,936 \\
\hline & Grapes & 24,620 & 36,566 & & & & \\
\hline & Apples & 24,061 & 29,271 & Fluometuron & Cotton & $4,500,857$ & $4,983,276$ \\
\hline & Almonds & 18,552 & 35,269 & & & & \\
\hline & Strawberries & 12,253 & 6,063 & Fomesafen & Soybeans & 540,173 & $1,970,733$ \\
\hline & Peaches & 10,977 & 22,227 & & Green Beans & 2,749 & 8,898 \\
\hline & Pears & 4,453 & 4,783 & & & & \\
\hline & Walnuts & 4,226 & 8,453 & Fonofos & Corn & $2,182,026$ & $2,098,413$ \\
\hline & Nectarines & 3,326 & 4,291 & & Peanuts & 289,569 & 148,245 \\
\hline & & & & & Potatoes & 88,102 & 37,438 \\
\hline \multirow[t]{5}{*}{ Fenoxaprop } & Soybeans & 359,022 & $3,346,290$ & & Mint & 62,928 & 30,696 \\
\hline & Wheat for Grain & 148,581 & $1,857,121$ & & Sweet Potatoes & 58,282 & 14,570 \\
\hline & Rice & 26,752 & 198,953 & & Sweet Corn & 29,505 & 21,497 \\
\hline & Peanuts & 7,641 & 50,943 & & Green Beans & 19,973 & 9,271 \\
\hline & Cotton & 1,642 & 8,917 & Fonofos & Sugar Beets for Sugar & 17,201 & 16,795 \\
\hline
\end{tabular}


Table 4. Primary crops that account for use of each pesticide, 1992-1995- Continued

\begin{tabular}{|c|c|c|c|c|c|c|c|}
\hline Compound & Crop & $\begin{array}{c}\text { Total } \\
\text { amount } \\
\text { applied } \\
\text { (pounds) }\end{array}$ & $\begin{array}{c}\text { Total area } \\
\text { treated } \\
\text { (acres) }\end{array}$ & Compound & Crop & $\begin{array}{c}\text { Total } \\
\text { amount } \\
\text { applied } \\
\text { (pounds) }\end{array}$ & $\begin{array}{c}\text { Total area } \\
\text { treated } \\
\text { (acres) }\end{array}$ \\
\hline \multirow[t]{2}{*}{ Fonofos } & Tobacco & 12,863 & 8,188 & Imazamethabenz & Sunflowers & 313 & 31,262 \\
\hline & Asparagus & 10,647 & 4,964 & & Corn & 262 & 8,731 \\
\hline \multirow[t]{8}{*}{ Formetanate $\mathrm{HCl}$} & All Citrus & 165,042 & 136,630 & Imazaquin & Soybeans & 807,270 & $8,634,943$ \\
\hline & Apples & 96,935 & 74,594 & & & & \\
\hline & Nectarines & 43,984 & 35,759 & Imazethapyr & Soybeans & $1,325,374$ & $21,971,760$ \\
\hline & Alfalfa Hay & 6,886 & 9,381 & & Corn & 21,889 & 364,819 \\
\hline & Pears & 6,683 & 8,583 & & Peanuts & 18,850 & 305,425 \\
\hline & Peaches & 6,221 & 6,891 & & Dry Beans & 7,479 & 14,957 \\
\hline & Plums & 2,359 & 2,609 & & Green Peas & 2,234 & 63,127 \\
\hline & & & & & Green Beans & 1,025 & 34,164 \\
\hline \multirow[t]{11}{*}{ Fosetyl-Al } & Lettuce & 24,695 & 92,868 & & Alfalfa Hay & 485 & 4,845 \\
\hline & All Citrus & 192,941 & 40,840 & & & & \\
\hline & Hops & 33,870 & 7,796 & Imidacloprid & Cotton & 34,676 & 799,497 \\
\hline & Cauliflower & 4,595 & 2,298 & & Potatoes & 26,291 & 158,228 \\
\hline & Onions & 3,593 & 1,843 & & & & \\
\hline & Spinach & 2,724 & 1,124 & Iprodione & Almonds & 332,375 & 273,335 \\
\hline & Collards & 1,043 & 498 & & Potatoes & 112,209 & 114,019 \\
\hline & Asparagus & 842 & 261 & & Grapes & 104,347 & 126,892 \\
\hline & Broccoli & 439 & 241 & & Rice & 99,401 & 180,285 \\
\hline & Avocados & 369 & 671 & & Peaches & 68,814 & 73,974 \\
\hline & & & & & Lettuce & 51,358 & 53,552 \\
\hline \multirow[t]{9}{*}{ Gibberellic acid } & Grapes & 26,471 & 374,525 & & Strawberries & 43,513 & 21,166 \\
\hline & All Citrus & 3,604 & 70,294 & & Cherries & 43,063 & 46,263 \\
\hline & Apples & 2,744 & 89,976 & & Onions & 30,934 & 28,948 \\
\hline & Cherries & 772 & 24,653 & & Carrots & 30,426 & 23,761 \\
\hline & Blueberries & 74 & 438 & & & & \\
\hline & Artichokes & 74 & 3,235 & Isopropalin & Tobacco & 139,291 & 139,275 \\
\hline & Hops & 20 & 1,314 & & & & \\
\hline & Carrots & 10 & 3,375 & Lactofen & Soybeans & 293,654 & $3,128,785$ \\
\hline & & & & & Cotton & 78,292 & 491,664 \\
\hline \multirow[t]{11}{*}{ Glyphosate } & Soybeans & $6,492,564$ & $10,386,426$ & & Tomatoes & 2,493 & 24,935 \\
\hline & Corn & $3,102,793$ & $4,463,460$ & & Green Beans & 2,057 & 2,743 \\
\hline & All Citrus & $1,139,922$ & 648,562 & & Sweet Peppers & 919 & 9,190 \\
\hline & All Pasture & 991,773 & $2,450,794$ & & & & \\
\hline & Wheat for Grain & 953,321 & $1,681,278$ & Lambdacyhalothrin & Cotton & 212,782 & $2,666,028$ \\
\hline & Cotton & 702,753 & $1,008,356$ & & Onions & 664 & 14,306 \\
\hline & Almonds & 476,132 & 440,863 & & & & \\
\hline & Grapes & 430,492 & 509,544 & Lindane & Pecans & 57,494 & 79,507 \\
\hline & Sorghum & 309,632 & 500,487 & & Squash & 6,272 & 7,433 \\
\hline & Rice & 203,008 & 204,477 & & Safflower & 3,110 & 1,681 \\
\hline & & & & & Sugar Beets for Sugar & 2,136 & 1,512 \\
\hline \multirow[t]{5}{*}{ Hexazinone } & Alfalfa Hay & 439,542 & 555,480 & & Lettuce & 636 & 1,957 \\
\hline & Blueberries & 15,186 & 13,538 & & Sweet Peppers & 291 & 579 \\
\hline & Field and Grass Seed & 6,012 & 11,809 & & Melons & 253 & 1,858 \\
\hline & Sugar Cane for Seed & 742 & 1,649 & & Cantaloupes & 119 & 515 \\
\hline & and Sugar & & & & Cauliflower & 118 & 460 \\
\hline \multirow[t]{2}{*}{ Imazamethabenz } & Wheat for Grain & 419,054 & $1,333,384$ & Linuron & Hot Peppers & 61 & 124 \\
\hline & Barley & 30,234 & 83,671 & & Soybeans & $1,573,478$ & $2,698,041$ \\
\hline
\end{tabular}


Table 4. Primary crops that account for use of each pesticide, 1992-1995- Continued

\begin{tabular}{|c|c|c|c|c|c|c|c|}
\hline Compound & Crop & $\begin{array}{c}\text { Total } \\
\text { amount } \\
\text { applied } \\
\text { (pounds) }\end{array}$ & $\begin{array}{c}\text { Total area } \\
\text { treated } \\
\text { (acres) }\end{array}$ & Compound & Crop & $\begin{array}{c}\text { Total } \\
\text { amount } \\
\text { applied } \\
\text { (pounds) }\end{array}$ & $\begin{array}{c}\text { Total area } \\
\text { treated } \\
\text { (acres) }\end{array}$ \\
\hline \multirow[t]{6}{*}{ Linuron } & Cotton & 216,174 & 265,648 & & Flax & 13,334 & 53,336 \\
\hline & Potatoes & 93,339 & 111,044 & & Dry Peas & 7,103 & 27,853 \\
\hline & Carrots & 73,568 & 54,531 & & Rye & 6,942 & 13,878 \\
\hline & Asparagus & 14,118 & 15,757 & & Green Peas & 6,636 & 31,193 \\
\hline & Celery & 4,155 & 4,462 & & & & \\
\hline & & & & МСРВ & Green Peas & 26,346 & 46,386 \\
\hline \multirow[t]{11}{*}{ Malathion } & Cotton & $1,289,790$ & 654,904 & & Sod & 17,301 & 19,245 \\
\hline & Alfalfa Hay & 627,335 & 449,864 & & Dry Peas & 9,982 & 10,938 \\
\hline & Sorghum & 231,109 & 176,281 & & & & \\
\hline & Wheat for Grain & 112,212 & 119,732 & MCPP & Field and Grass Seed & 629 & 524 \\
\hline & Rice & 98,241 & 122,265 & & & & \\
\hline & Strawberries & 91,347 & 13,081 & Mepiquat chloride & Cotton & 110,871 & $2,854,066$ \\
\hline & Apples & 76,804 & 54,979 & & & & \\
\hline & Cherries & 72,032 & 18,959 & Metalaxyl & Tobacco & 394,861 & 759,847 \\
\hline & Blueberries & 57,124 & 18,837 & & Potatoes & 110,363 & 347,176 \\
\hline & Barley & 33,837 & 35,696 & & All Citrus & 104,307 & 76,304 \\
\hline & & & & & Cotton & 64,455 & 790,181 \\
\hline \multirow[t]{4}{*}{ Maleic hydrazide } & Tobacco & $1,900,910$ & 613,197 & & Tomatoes & 38,245 & 87,294 \\
\hline & Potatoes & 181,300 & 81,165 & & Onions & 22,424 & 64,838 \\
\hline & Onions & 43,367 & 27,069 & & Watermelons & 16,932 & 32,308 \\
\hline & & & & & Peanuts & 16,251 & 32,501 \\
\hline \multirow[t]{11}{*}{ Mancozeb } & Potatoes & $2,275,952$ & 553,681 & & Lettuce & 16,214 & 43,069 \\
\hline & Tomatoes & $1,431,080$ & 125,752 & & Apples & 16,105 & 5,404 \\
\hline & Apples & $1,202,774$ & 210,878 & & & & \\
\hline & Wheat for Grain & 688,551 & 426,818 & Metaldehyde & All Citrus & 29,785 & 48,350 \\
\hline & Sweet Corn & 482,144 & 132,725 & & Field and Grass Seed & 16,445 & 22,841 \\
\hline & Watermelons & 378,949 & 60,106 & & Strawberries & 4,202 & 3,494 \\
\hline & Grapes & 342,661 & 125,165 & & Artichokes & 3,910 & 3,720 \\
\hline & Squash & 282,468 & 30,273 & & Raspberries & 764 & 624 \\
\hline & Cotton & 242,627 & 115,967 & & Avocados & 572 & 1,342 \\
\hline & Cucumbers & 233,671 & 28,494 & & Cauliflower & 451 & 418 \\
\hline & & & & & Mint & 394 & 464 \\
\hline \multirow[t]{11}{*}{ Maneb } & Potatoes & 874,158 & 203,667 & & Sweet Peppers & 143 & 386 \\
\hline & Tomatoes & 470,344 & 64,428 & & Green Beans & 95 & 249 \\
\hline & Lettuce & 429,769 & 135,085 & & & & \\
\hline & Sweet Peppers & 242,259 & 24,192 & Metam sodium & Potatoes & $22,405,286$ & 147,207 \\
\hline & Sweet Corn & 221,487 & 59,400 & & Peanuts & $3,696,806$ & 116,252 \\
\hline & Almonds & 184,669 & 61,721 & & Tomatoes & $2,236,921$ & 63,172 \\
\hline & Pumpkins & 125,087 & 13,703 & & Cotton & $1,260,255$ & 31,913 \\
\hline & Onions & 102,982 & 19,469 & & Onions & 482,475 & 3,390 \\
\hline & Watermelons & 98,655 & 19,913 & & Carrots & 467,638 & 3,325 \\
\hline & Cabbage & 68,694 & 15,149 & & Lettuce & 339,194 & 1,957 \\
\hline & & & & & Brussel Sprouts & 242,152 & 3,354 \\
\hline \multirow[t]{6}{*}{ MCPA } & Wheat for Grain & $3,445,394$ & $9,352,843$ & & Cantaloupes & 184,965 & 2,059 \\
\hline & Barley & 633,976 & $1,680,914$ & & Watermelons & 134,103 & 1,538 \\
\hline & All Pasture & 225,232 & 416,107 & & & & \\
\hline & Oats & 207,439 & 548,897 & Methamidophos & Potatoes & 376,011 & 283,748 \\
\hline & Rice & 60,022 & 75,416 & & Cotton & 309,752 & 391,634 \\
\hline & Field and Grass Seed & 51,260 & 114,985 & & Tomatoes & 242,236 & 90,473 \\
\hline
\end{tabular}


Table 4. Primary crops that account for use of each pesticide, 1992-1995- Continued

\begin{tabular}{|c|c|c|c|c|c|c|c|}
\hline Compound & Crop & $\begin{array}{c}\text { Total } \\
\text { amount } \\
\text { applied } \\
\text { (pounds) }\end{array}$ & $\begin{array}{c}\text { Total area } \\
\text { treated } \\
\text { (acres) }\end{array}$ & Compound & Crop & $\begin{array}{c}\text { Total } \\
\text { amount } \\
\text { applied } \\
\text { (pounds) }\end{array}$ & $\begin{array}{c}\text { Total area } \\
\text { treated } \\
\text { (acres) }\end{array}$ \\
\hline \multirow[t]{9}{*}{ Methamidophos } & Sugar Beets for & 43,255 & 34,771 & \multirow[t]{3}{*}{ Methyl bromide } & Nectarines & 478,112 & 2,146 \\
\hline & Sugar & & & & Cucumbers & 406,988 & 2,236 \\
\hline & Watermelons & 34,093 & 13,735 & & & & \\
\hline & Alfalfa Hay & 25,085 & 18,762 & \multirow[t]{11}{*}{ Methyl parathion } & Cotton & $5,899,626$ & $2,788,735$ \\
\hline & Cabbage & 20,770 & 17,205 & & Corn & $1,014,112$ & $1,850,090$ \\
\hline & Broccoli & 18,074 & 15,611 & & Alfalfa Hay & 396,642 & 498,640 \\
\hline & Lettuce & 8,828 & 9,862 & & Wheat for Grain & 330,999 & 763,679 \\
\hline & Eggplant & 5,666 & 1,061 & & Rice & 267,585 & 519,167 \\
\hline & \multirow{3}{*}{ Cotton } & \multirow{3}{*}{491,795} & \multirow{3}{*}{859,590} & & Soybeans & 220,688 & 453,360 \\
\hline \multirow[t]{2}{*}{ Methazole } & & & & & Apples & 208,979 & 126,939 \\
\hline & & & & & Sunflowers & 199,057 & 124,474 \\
\hline \multirow[t]{11}{*}{ Methidathion } & All Citrus & 159,771 & 59,319 & & Peaches & 115,432 & 56,584 \\
\hline & Almonds & 91,700 & 70,538 & & Sweet Corn & 50,466 & 67,996 \\
\hline & Plums & 63,607 & 41,983 & & & & \\
\hline & Peaches & 36,225 & 25,332 & \multirow[t]{3}{*}{ Metiram } & Apples & 614,448 & 69,228 \\
\hline & Safflower & 35,403 & 60,518 & & Potatoes & 307,696 & 57,893 \\
\hline & Walnuts & 35,286 & 23,245 & & & & \\
\hline & Artichokes & 16,904 & 8,088 & \multirow[t]{11}{*}{ Metolachlor } & Corn & $41,546,112$ & $21,901,483$ \\
\hline & Apples & 14,550 & 17,204 & & Soybeans & $9,916,637$ & $5,195,717$ \\
\hline & Alfalfa Hay & 14,071 & 28,143 & & Sorghum & $3,089,336$ & $2,250,997$ \\
\hline & Nectarines & 12,676 & 7,509 & & Peanuts & $1,319,308$ & 651,241 \\
\hline & & & & & Cotton & 602,112 & 492,953 \\
\hline \multirow[t]{10}{*}{ Methomyl } & Cotton & 409,821 & 875,885 & & Sweet Corn & 494,057 & 230,905 \\
\hline & Sweet Corn & 315,003 & 171,008 & & Potatoes & 357,695 & 193,345 \\
\hline & Lettuce & 247,866 & 190,100 & & Dry Beans & 351,784 & 188,490 \\
\hline & Apples & 153,951 & 139,278 & & Green Beans & 175,669 & 112,500 \\
\hline & Alfalfa Hay & 122,379 & 192,585 & & Green Peas & 33,228 & 19,125 \\
\hline & Corn & 116,553 & 248,365 & & & & \\
\hline & Peanuts & 99,489 & 248,722 & \multirow[t]{11}{*}{ Metribuzin } & Soybeans & $1,707,148$ & $6,119,359$ \\
\hline & Tomatoes & 94,352 & 90,409 & & Potatoes & 377,256 & 779,416 \\
\hline & Sorghum & 88,785 & 224,629 & & Alfalfa Hay & 215,321 & 348,321 \\
\hline & Grapes & 75,880 & 100,640 & & $\begin{array}{l}\text { Sugar Cane for Seed } \\
\text { and Sugar }\end{array}$ & 189,811 & 145,324 \\
\hline \multirow[t]{9}{*}{ Methoxychlor } & Apples & 73,866 & 52,387 & & Wheat for Grain & 82,793 & 417,650 \\
\hline & Squash & 4,764 & 3,811 & & Tomatoes & 48,913 & 87,892 \\
\hline & Field and Grass Seed & 4,284 & 4,189 & & Field and Grass Seed & 29,704 & 65,288 \\
\hline & Grapes & 2,738 & 401 & & Corn & 26,825 & 383,208 \\
\hline & Green Peas & 1,181 & 653 & & Asparagus & 14,377 & 13,995 \\
\hline & Strawberries & 489 & 220 & & Dry Peas & 8,574 & 25,654 \\
\hline & Dry Peas & 235 & 117 & & & & \\
\hline & Blueberries & 98 & 50 & Metsulfuron & Wheat for Grain & 34,589 & $7,511,573$ \\
\hline & & & & & Barley & 945 & 236,351 \\
\hline \multirow[t]{8}{*}{ Methyl bromide } & Tomatoes & $13,035,891$ & 70,359 & & & & \\
\hline & Tobacco & $5,603,849$ & 12,534 & \multirow[t]{7}{*}{ Mevinphos } & Lettuce & 101,986 & 118,207 \\
\hline & Strawberries & $5,529,716$ & 26,936 & & Broccoli & 19,642 & 21,913 \\
\hline & Watermelons & $3,748,578$ & 18,025 & & Collards & 12,048 & 5,745 \\
\hline & Sweet Peppers & $3,140,497$ & 16,608 & & Cabbage & 11,338 & 8,137 \\
\hline & Grapes & $2,666,467$ & 7,285 & & Cauliflower & 7,028 & 8,475 \\
\hline & Almonds & $1,697,323$ & 22,043 & & Squash & 7,003 & 8,497 \\
\hline & Carrots & 607,371 & 3,325 & & Sweet Peppers & 6,779 & 5,592 \\
\hline
\end{tabular}


Table 4. Primary crops that account for use of each pesticide, 1992-1995- Continued

\begin{tabular}{|c|c|c|c|c|c|c|c|}
\hline Compound & Crop & $\begin{array}{l}\text { Total } \\
\text { amount } \\
\text { applied } \\
\text { (pounds) }\end{array}$ & $\begin{array}{c}\text { Total area } \\
\text { treated } \\
\text { (acres) }\end{array}$ & Compound & Crop & $\begin{array}{c}\text { Total } \\
\text { amount } \\
\text { applied } \\
\text { (pounds) }\end{array}$ & $\begin{array}{c}\text { Total area } \\
\text { treated } \\
\text { (acres) }\end{array}$ \\
\hline \multirow[t]{3}{*}{ Mevinphos } & Alfalfa Hay & 6,675 & 20,162 & $\overline{\text { Naptalam }}$ & Melons & 782 & 612 \\
\hline & Green Peas & 6,318 & 17,592 & & & & \\
\hline & Sweet Corn & 5,861 & 11,907 & Nicosulfuron & Corn & 284,159 & $9,821,302$ \\
\hline \multirow[t]{2}{*}{ Molinate } & Rice & $4,903,206$ & $1,420,255$ & Norflurazon & Cotton & $1,573,179$ & $2,030,640$ \\
\hline & & & & & All Citrus & 417,879 & 122,686 \\
\hline \multirow[t]{4}{*}{ MSMA } & Cotton & $5,737,271$ & $3,793,231$ & & Almonds & 55,637 & 44,086 \\
\hline & Sod & 14,555 & 6,350 & & Peanuts & 52,403 & 56,347 \\
\hline & All Citrus & 11,543 & 7,874 & & Grapes & 48,272 & 49,694 \\
\hline & & & & & Apples & 31,581 & 30,771 \\
\hline \multirow[t]{8}{*}{ Myclobutanil } & Grapes & 213,321 & 735,200 & & Blueberries & 16,833 & 4,676 \\
\hline & Apples & 74,972 & 293,780 & & Plums & 14,674 & 11,879 \\
\hline & Cherries & 12,203 & 55,060 & & Peaches & 14,012 & 12,286 \\
\hline & Nectarines & 7,125 & 8,940 & & Asparagus & 11,463 & 5,879 \\
\hline & Peaches & 3,673 & 27,695 & & & & \\
\hline & Tomatoes & 3,613 & 32,849 & Oil & All Citrus & $20,100,541$ & 367,576 \\
\hline & Strawberries & 2,905 & 13,204 & & Apples & $13,827,892$ & 407,562 \\
\hline & & & & & Almonds & $6,070,684$ & 224,840 \\
\hline \multirow[t]{4}{*}{ NAA } & Apples & 10,938 & 195,507 & & Pears & $5,795,382$ & 74,803 \\
\hline & Pears & 2,651 & 39,364 & & Plums & $2,387,612$ & 89,179 \\
\hline & Olives & 76 & 355 & & Peaches & $2,123,019$ & 75,286 \\
\hline & & & & & Cherries & 966,923 & 25,385 \\
\hline \multirow[t]{2}{*}{ NAD } & Apples & 3,974 & 51,143 & & Nectarines & 956,219 & 26,462 \\
\hline & & & & & Olives & 299,827 & 5,686 \\
\hline \multirow[t]{11}{*}{ Naled } & Cotton & 153,184 & 159,566 & & Apricots & 268,147 & 10,748 \\
\hline & Safflower & 24,557 & 36,983 & & & & \\
\hline & All Citrus & 22,817 & 19,806 & Oryzalin & Grapes & 206,036 & 112,884 \\
\hline & Grapes & 19,904 & 29,142 & & Almonds & 179,026 & 105,807 \\
\hline & Alfalfa Hay & 16,390 & 14,967 & & All Citrus & 94,664 & 31,772 \\
\hline & Broccoli & 10,880 & 7,772 & & Pistachios & 62,646 & 24,012 \\
\hline & Sugar Beets for Sugar & 8,665 & 10,080 & & Apples & 42,676 & 26,798 \\
\hline & Field and Grass Seed & 5,878 & 11,995 & & Peaches & 35,658 & 32,608 \\
\hline & Brussel Sprouts & 5,185 & 3,744 & & Pecans & 32,921 & 70,070 \\
\hline & Cabbage & 5,068 & 3,325 & & Plums & 32,338 & 17,419 \\
\hline & & & & & Cherries & 19,644 & 10,365 \\
\hline \multirow[t]{11}{*}{ Napropamide } & Tobacco & 198,830 & 180,407 & & Pears & 17,213 & 11,677 \\
\hline & Tomatoes & 84,867 & 75,940 & & & & \\
\hline & Cranberries & 44,513 & 10,309 & Oxamyl & Cotton & 477,086 & $1,328,662$ \\
\hline & Strawberries & 33,340 & 10,017 & & Potatoes & 75,625 & 77,547 \\
\hline & Sweet Potatoes & 17,220 & 17,220 & & Apples & 72,819 & 122,200 \\
\hline & Almonds & 16,779 & 8,817 & & Mint & 61,554 & 48,468 \\
\hline & Sweet Peppers & 16,772 & 14,040 & & Sweet Peppers & 27,967 & 13,362 \\
\hline & Grapes & 12,929 & 7,739 & & Celery & 23,599 & 18,904 \\
\hline & Hot Peppers & 8,904 & 6,544 & & Tomatoes & 21,537 & 21,289 \\
\hline & Peaches & 4,606 & 2,507 & & Cucumbers & 12,269 & 9,139 \\
\hline & & & & & Onions & 11,075 & 8,157 \\
\hline \multirow[t]{4}{*}{ Naptalam } & Cucumbers & 61,484 & 24,919 & Oxamyl & Melons & 9,379 & 14,260 \\
\hline & Watermelons & 25,840 & 11,914 & & & & \\
\hline & Cantaloupes & 10,425 & 6,013 & Oxydemeton & Cotton & 96,333 & 210,348 \\
\hline & Pumpkins & 932 & 173 & Methyl & Broccoli & 41,573 & 66,379 \\
\hline
\end{tabular}


Table 4. Primary crops that account for use of each pesticide, 1992-1995- Continued

\begin{tabular}{|c|c|c|c|c|c|c|c|}
\hline Compound & Crop & $\begin{array}{c}\text { Total } \\
\text { amount } \\
\text { applied } \\
\text { (pounds) }\end{array}$ & $\begin{array}{c}\text { Total area } \\
\text { treated } \\
\text { (acres) }\end{array}$ & Compound & Crop & $\begin{array}{c}\text { Total } \\
\text { amount } \\
\text { applied } \\
\text { (pounds) }\end{array}$ & $\begin{array}{c}\text { Total area } \\
\text { treated } \\
\text { (acres) }\end{array}$ \\
\hline \multirow{10}{*}{$\begin{array}{l}\text { Oxydemeton } \\
\text { Methyl }\end{array}$} & Cauliflower & 28,803 & 35,485 & $\overline{\mathrm{PCNB}}$ & Dry Beans & 602 & 7,528 \\
\hline & & & & & Onions & 400 & 67 \\
\hline & Field Grass and Seed & 15,674 & 31,988 & & Dry Peas & 0 & 25 \\
\hline & Mint & 14,687 & 18,305 & & & & \\
\hline & Sugar Beets for Sugar & 7,763 & 14,615 & Pebulate & Tobacco & 438,651 & 111,987 \\
\hline & Cabbage & 7,268 & 7,773 & & Tomatoes & 105,522 & 40,683 \\
\hline & Sorghum & 4,753 & 9,511 & & Sugar Beets for Sugar & 16,300 & 4,535 \\
\hline & Brussel Sprouts & 4,329 & 3,900 & & & & \\
\hline & Sweet Peppers & 3,171 & 5,984 & Pendimethalin & Soybeans & $12,728,006$ & $13,629,471$ \\
\hline & & & & & Corn & $3,957,004$ & $3,269,047$ \\
\hline \multirow[t]{11}{*}{ Oxyfluorfen } & Grapes & 102,702 & 144,720 & & Cotton & $1,986,008$ & $2,595,719$ \\
\hline & Almonds & 95,932 & 282,152 & & Peanuts & 479,612 & 506,696 \\
\hline & Cotton & 77,944 & 380,376 & & Sugar Cane for Seed & 375,676 & 187,838 \\
\hline & Apples & 37,006 & 23,945 & & and Sugar & & \\
\hline & Pistachios & 26,385 & 25,346 & & Tobacco & 336,278 & 418,361 \\
\hline & Field and Grass Seed & 22,841 & 114,203 & & Potatoes & 242,424 & 256,402 \\
\hline & Olives & 22,441 & 6,397 & & Rice & 198,661 & 276,579 \\
\hline & Onions & 16,110 & 76,704 & & Sunflowers & 149,703 & 134,880 \\
\hline & Plums & 15,092 & 30,005 & & Onions & 85,056 & 34,326 \\
\hline & Walnuts & 12,552 & 46,490 & & & & \\
\hline & & & & Permethrin & Corn & 405,729 & $3,572,476$ \\
\hline \multirow[t]{4}{*}{ Oxytetracycline } & Pears & 17,638 & 45,258 & & Wheat for Grain & 160,196 & $1,607,085$ \\
\hline & Peaches & 17,292 & 23,394 & & Alfalfa Hay & 156,160 & 748,047 \\
\hline & Apples & 390 & 2,555 & & Sweet Corn & 111,657 & 288,275 \\
\hline & & & & & Lettuce & 48,706 & 215,036 \\
\hline \multirow[t]{7}{*}{ Oxythioquinox } & All Citrus & 84,925 & 115,328 & & Tomatoes & 43,622 & 39,868 \\
\hline & Pears & 32,095 & 28,418 & & Cotton & 42,091 & 293,441 \\
\hline & Apples & 26,159 & 58,942 & & Potatoes & 42,065 & 242,600 \\
\hline & Walnuts & 1,226 & 2,113 & & Onions & 23,295 & 38,491 \\
\hline & Strawberries & 321 & 1,340 & & Peaches & 21,576 & 78,275 \\
\hline & Apricots & 147 & 239 & & & & \\
\hline & & & & Phenmedipham & Sugar Beets for Sugar & 180,624 & 946,995 \\
\hline \multirow[t]{11}{*}{ Paraquat } & Corn & $1,240,472$ & $2,298,051$ & & Beets & 1,199 & 1,423 \\
\hline & Soybeans & $1,222,533$ & $2,282,829$ & & Spinach & 704 & 1,127 \\
\hline & Cotton & 634,304 & $2,020,983$ & & Field and Grass Seed & 640 & 3,998 \\
\hline & Alfalfa Hay & 112,891 & 204,360 & & & & \\
\hline & Peanuts & 110,997 & 889,524 & Phorate & Corn & $1,696,959$ & $1,518,797$ \\
\hline & Sorghum & 106,352 & 425,410 & & Potatoes & 838,166 & 318,365 \\
\hline & Grapes & 98,385 & 167,807 & & Cotton & 414,009 & 587,994 \\
\hline & Almonds & 79,250 & 123,442 & & Sugar Cane for Seed & 329,645 & 84,524 \\
\hline & Apples & 74,096 & 129,720 & & and Sugar & & \\
\hline & All Citrus & 66,571 & 86,693 & & Wheat for Grain & 161,429 & 167,763 \\
\hline & & & & & Peanuts & 150,613 & 135,687 \\
\hline \multirow[t]{7}{*}{ PCNB } & Cotton & $1,207,448$ & $1,476,625$ & & Sorghum & 117,781 & 106,147 \\
\hline & Peanuts & 447,997 & 89,599 & & Sugar Beets for Sugar & 46,572 & 41,653 \\
\hline & Cabbage & 11,467 & 2,333 & & Sweet Corn & 30,951 & 24,358 \\
\hline & Brussel Sprouts & 11,093 & 507 & Phorate & Green Beans & 17,053 & 12,677 \\
\hline & Broccoli & 8,490 & 1,943 & & & & \\
\hline & Green Beans & 5,427 & 7,161 & Phosmet & Apples & 506,042 & 155,668 \\
\hline & Cauliflower & 2,070 & 662 & & Pears & 95,769 & 26,648 \\
\hline
\end{tabular}


Table 4. Primary crops that account for use of each pesticide, 1992-1995- Continued

\begin{tabular}{|c|c|c|c|c|c|c|c|}
\hline Compound & Crop & $\begin{array}{c}\text { Total } \\
\text { amount } \\
\text { applied } \\
\text { (pounds) }\end{array}$ & $\begin{array}{c}\text { Total area } \\
\text { treated } \\
\text { (acres) }\end{array}$ & Compound & Crop & $\begin{array}{c}\text { Total } \\
\text { amount } \\
\text { applied } \\
\text { (pounds) }\end{array}$ & $\begin{array}{c}\text { Total area } \\
\text { treated } \\
\text { (acres) }\end{array}$ \\
\hline \multirow[t]{9}{*}{ Phosmet } & Alfalfa Hay & 87,319 & 115,090 & Propargite & Mint & 136,235 & 67,048 \\
\hline & Peaches & 81,368 & 27,694 & & Alfalfa Hay & 94,615 & 54,832 \\
\hline & Almonds & 44,483 & 8,817 & & Potatoes & 88,857 & 43,039 \\
\hline & Potatoes & 28,701 & 29,092 & & Dry Beans & 85,114 & 65,232 \\
\hline & Cherries & 20,381 & 15,330 & & & & \\
\hline & Plums & 15,757 & 6,430 & Propiconazole & Wheat for Grain & 103,381 & 913,126 \\
\hline & Green Peas & 13,464 & 17,952 & & Rice & 76,949 & 281,918 \\
\hline & Grapes & 11,548 & 9,545 & & Field and Grass Seed & 47,582 & 339,872 \\
\hline & & & & & Pecans & 34,391 & 134,932 \\
\hline \multirow[t]{6}{*}{ Picloram } & All Pasture & $1,583,553$ & $6,620,303$ & & Barley & 12,386 & 95,280 \\
\hline & Other Hay & 113,067 & 144,198 & & Sweet Corn & 10,940 & 46,289 \\
\hline & Wheat for Grain & 21,040 & 300,576 & & Peaches & 4,208 & 19,569 \\
\hline & Flax & 3,336 & 6,673 & & Wild Rice & 830 & 7,545 \\
\hline & Oats & 1,907 & 19,075 & & Celery & 463 & 2,257 \\
\hline & Barley & 256 & 12,819 & & Rye & 212 & 3,023 \\
\hline \multirow[t]{2}{*}{ Primisulfuron } & Corn & 64,406 & $2,192,931$ & Pyrazon & Sugar Beets for Sugar & 299,491 & 201,139 \\
\hline & & & & & Beets & 16,934 & 5,016 \\
\hline Profenofos & Cotton & $2,121,560$ & $1,695,563$ & & Field and Grass Seed & 4,798 & 3,998 \\
\hline \multirow[t]{4}{*}{ Prometryn } & Cotton & $1,821,722$ & $2,540,598$ & Pyridate & Peanuts & 80,327 & 86,373 \\
\hline & Celery & 20,617 & 16,894 & & Cabbage & 589 & 621 \\
\hline & Parsley & 2,962 & 1,551 & & & & \\
\hline & & & & Quinclorac & Rice & 126,968 & 342,392 \\
\hline \multirow[t]{10}{*}{ Pronamide } & Lettuce & 131,865 & 149,126 & & & & \\
\hline & Alfalfa Hay & 27,797 & 34,231 & Quizalofop & Soybeans & 191,023 & $3,611,894$ \\
\hline & Field and Grass Seed & 23,948 & 36,117 & & & & \\
\hline & Pears & 16,534 & 5,101 & Sethoxydim & Soybeans & 737,842 & $3,991,533$ \\
\hline & Artichokes & 1,372 & 1,213 & & Cotton & 266,883 & $1,009,322$ \\
\hline & Raspberries & 569 & 739 & & Sugar Beets for Sugar & 97,363 & 476,070 \\
\hline & Apples & 258 & 663 & & Peanuts & 70,423 & 251,512 \\
\hline & Blackberries & 254 & 416 & & Alfalfa Hay & 48,318 & 187,412 \\
\hline & Blueberries & 65 & 109 & & Potatoes & 17,572 & 77,632 \\
\hline & & & & & Mint & 14,043 & 51,751 \\
\hline \multirow[t]{5}{*}{ Propachlor } & Corn & $2,224,304$ & 940,811 & & Dry Beans & 7,934 & 39,480 \\
\hline & Sorghum & $1,620,434$ & 724,478 & & Sunflowers & 7,392 & 63,612 \\
\hline & Sweet Corn & 12,011 & 6,355 & & Tomatoes & 5,313 & 31,847 \\
\hline & Green Peas & 8,784 & 3,231 & & & & \\
\hline & & & & Siduron & Sod & 247 & 62 \\
\hline \multirow[t]{2}{*}{ Propamocarb } & Potatoes & 54,198 & 55,337 & & & & \\
\hline & & & & Simazine & Corn & $2,349,911$ & $1,964,438$ \\
\hline \multirow[t]{2}{*}{ Propanil } & Rice & $7,229,829$ & $2,337,989$ & & All Citrus & $1,140,321$ & 350,409 \\
\hline & & & & & Grapes & 481,199 & 314,580 \\
\hline \multirow[t]{7}{*}{ Propargite } & Corn & 839,821 & 495,451 & & Alfalfa Hay & 333,372 & 347,681 \\
\hline & Grapes & 433,724 & 269,561 & & Apples & 114,112 & 100,610 \\
\hline & Cotton & 420,263 & 240,841 & & Peaches & 61,784 & 50,069 \\
\hline & Almonds & 392,007 & 202,797 & & Almonds & 59,940 & 96,990 \\
\hline & Apples & 330,626 & 154,788 & & Walnuts & 39,749 & 31,698 \\
\hline & Peanuts & 183,876 & 114,923 & & Pecans & 32,490 & 21,784 \\
\hline & & & & & Asparagus & 31,155 & 20,454 \\
\hline
\end{tabular}


Table 4. Primary crops that account for use of each pesticide, 1992-1995- Continued

\begin{tabular}{|c|c|c|c|c|c|c|c|}
\hline Compound & Crop & $\begin{array}{c}\text { Total } \\
\text { amount } \\
\text { applied } \\
\text { (pounds) }\end{array}$ & $\begin{array}{c}\text { Total area } \\
\text { treated } \\
\text { (acres) }\end{array}$ & Compound & Crop & $\begin{array}{c}\text { Total } \\
\text { amount } \\
\text { applied } \\
\text { (pounds) }\end{array}$ & $\begin{array}{c}\text { Total area } \\
\text { treated } \\
\text { (acres) }\end{array}$ \\
\hline \multirow[t]{9}{*}{ Sodium chlorate } & Cotton & $4,581,793$ & $1,507,850$ & Terbufos & Sorghum & 181,752 & 247,071 \\
\hline & Dry Beans & 564,725 & 188,241 & & Sweet Corn & 48,529 & 47,370 \\
\hline & Hot Peppers & 33,504 & 4,171 & & & & \\
\hline & Safflower & 29,856 & 5,043 & Thiabendazole & Wheat for Grain & 159,142 & 289,350 \\
\hline & Rice & 19,606 & 4,005 & & & & \\
\hline & Sunflowers & 10,091 & 1,771 & Thidiazuron & Cotton & 452,393 & $2,717,045$ \\
\hline & Sweet Corn & 3,303 & 634 & & & & \\
\hline & Sorghum & 856 & 154 & Thifensulfuron & Wheat for Grain & 56,660 & $4,715,217$ \\
\hline & & & & & Soybeans & 18,689 & $6,248,335$ \\
\hline \multirow[t]{3}{*}{ Streptomycin } & Apples & 90,256 & 140,562 & & Barley & 7,631 & 685,164 \\
\hline & Pears & 18,187 & 44,865 & & & & \\
\hline & Onions & 36 & 200 & Thiobencarb & Rice & $1,436,364$ & 473,210 \\
\hline \multirow[t]{11}{*}{ Sulfur } & Grapes & $51,130,764$ & 756,273 & Thiodicarb & Cotton & $1,172,802$ & $1,664,837$ \\
\hline & Tomatoes & $7,203,079$ & 154,171 & & Sweet Corn & 169,413 & 69,190 \\
\hline & Sugar Beets for Sugar & $7,152,381$ & 264,056 & & Soybeans & 141,175 & 225,386 \\
\hline & Peaches & $5,700,305$ & 163,609 & & Cauliflower & 2,151 & 2,757 \\
\hline & All Citrus & $2,631,665$ & 98,725 & & Cabbage & 1,935 & 1,324 \\
\hline & Apples & $2,441,969$ & 130,194 & & Broccoli & 597 & 694 \\
\hline & Peanuts & $1,500,906$ & 294,181 & & & & \\
\hline & Cherries & $1,270,414$ & 63,735 & Thiophanate- & Apples & 105,174 & 99,667 \\
\hline & Pecans & $1,093,392$ & 99,237 & methyl & Soybeans & 100,526 & 167,544 \\
\hline & Dates & 864,919 & 5,881 & & Peaches & 89,745 & 55,810 \\
\hline & & & & & Wheat for Grain & 52,144 & 86,906 \\
\hline \multirow[t]{2}{*}{ Sulfuric acid } & Potatoes & $25,941,349$ & 105,608 & & Dry Beans & 49,026 & 48,373 \\
\hline & & & & & Almonds & 35,401 & 48,495 \\
\hline \multirow[t]{2}{*}{ Sulprofos } & Cotton & 842,478 & 530,154 & & Pecans & 24,296 & 29,630 \\
\hline & & & & & Green Beans & 12,965 & 9,815 \\
\hline \multirow[t]{2}{*}{ Tebufenozide } & Cotton & 42,104 & 290,444 & & Peanuts & 9,125 & 6,156 \\
\hline & & & & & Strawberries & 6,760 & 4,751 \\
\hline \multirow[t]{2}{*}{ Tebuthiuron } & All Pasture & 104,591 & 209,183 & & & & \\
\hline & & & & Thiram & Apples & 139,755 & 30,116 \\
\hline \multirow[t]{2}{*}{ Tefluthrin } & Corn & 423,845 & $3,561,133$ & & Strawberries & 117,301 & 15,007 \\
\hline & Sweet Corn & 128 & 1,067 & & Peaches & 9,320 & 1,353 \\
\hline \multirow[t]{8}{*}{ Terbacil } & Mint & 120,298 & 123,124 & Tralomethrin & Cotton & 42,376 & 765,264 \\
\hline & $\begin{array}{l}\text { Sugar Cane for Seed } \\
\text { and Sugar }\end{array}$ & 59,169 & 52,595 & & Soybeans & 10,955 & 547,744 \\
\hline & Alfalfa Hay & 35,795 & 66,080 & Triadimefon & Wheat for Grain & 45,990 & 437,375 \\
\hline & Apples & 30,348 & 41,159 & & Grapes & 30,285 & 160,456 \\
\hline & Peaches & 19,160 & 23,097 & & Sugar Beets for Sugar & 14,454 & 15,003 \\
\hline & Field and Grass Seed & 6,920 & 11,477 & & Apples & 10,926 & 62,926 \\
\hline & Blueberries & 6,474 & 8,748 & & Tomatoes & 3,942 & 32,849 \\
\hline & Strawberries & 2,710 & 5,154 & & Cantaloupes & 1,906 & 17,605 \\
\hline \multirow[t]{3}{*}{ Terbacil } & Asparagus & 1,591 & 2,628 & Triadimefon & Watermelons & 1,712 & 12,915 \\
\hline & Pecans & 1,242 & 1,657 & & Pears & 1,434 & 7,417 \\
\hline & & & & & Pumpkins & 808 & 4,728 \\
\hline \multirow[t]{2}{*}{ Terbufos } & Corn & $6,497,298$ & $5,949,434$ & & Sweet Peppers & 558 & 3,281 \\
\hline & Sugar Beets for Sugar & 472,498 & 432,038 & & & & \\
\hline
\end{tabular}


Table 4. Primary crops that account for use of each pesticide, 1992-1995- Continued

\begin{tabular}{|c|c|c|c|c|c|c|c|}
\hline Compound & Crop & $\begin{array}{c}\text { Total } \\
\text { amount } \\
\text { applied } \\
\text { (pounds) }\end{array}$ & $\begin{array}{c}\text { Total area } \\
\text { treated } \\
\text { (acres) }\end{array}$ & Compound & Crop & $\begin{array}{c}\text { Total } \\
\text { amount } \\
\text { applied } \\
\text { (pounds) }\end{array}$ & $\begin{array}{c}\text { Total area } \\
\text { treated } \\
\text { (acres) }\end{array}$ \\
\hline \multirow[t]{7}{*}{ Triallate } & Wheat for Grain & $1,821,278$ & $1,788,445$ & Triforine & Peaches & 61,996 & 67,125 \\
\hline & Barley & 390,850 & 375,954 & & Blueberries & 12,797 & 19,928 \\
\hline & Dry Peas & 39,678 & 31,743 & & Plums & 11,974 & 22,896 \\
\hline & Green Peas & 27,519 & 24,010 & & Nectarines & 5,514 & 10,728 \\
\hline & Flax & 4,004 & 4,004 & & Apples & 3,374 & 6,831 \\
\hline & Canola & 336 & 240 & Triforine & Cherries & 2,773 & 6,879 \\
\hline & & & & & Cranberries & 2,604 & 2,604 \\
\hline \multirow[t]{2}{*}{ Triasulfuron } & Wheat for Grain & 31,369 & $2,762,515$ & & Apricots & 1,776 & 3,344 \\
\hline & & & & & Asparagus & 682 & 3,130 \\
\hline \multirow[t]{2}{*}{ Tribenuron } & Wheat for Grain & 46,571 & $5,846,187$ & & & & \\
\hline & Barley & 2,929 & 371,924 & Trimethacarb & Corn & 151,150 & 151,150 \\
\hline \multirow[t]{2}{*}{ Tribufos } & Cotton & $3,721,092$ & $3,978,201$ & Triphenyltin & Pecans & 188,252 & 253,402 \\
\hline & & & & hydroxide & Sugar Beets for Sugar & 161,526 & 404,008 \\
\hline \multirow[t]{7}{*}{ Trichlorfon } & Other Hay & 6,184 & 6,184 & & Potatoes & 60,173 & 218,045 \\
\hline & Alfalfa Hay & 1,886 & 10,478 & & & & \\
\hline & Corn & 1,278 & 1,704 & Vernolate & Peanuts & 489,052 & 254,429 \\
\hline & Tobacco & 756 & 756 & & Sweet Corn & 9,635 & 3,300 \\
\hline & Sod & 212 & 78 & & & & \\
\hline & Cotton & 41 & 406 & Vinclozolin & Lettuce & 50,820 & 37,841 \\
\hline & & & & & Green Beans & 23,436 & 39,710 \\
\hline \multirow[t]{5}{*}{ Triclopyr } & All Pasture & 90,070 & 180,141 & & Strawberries & 21,688 & 15,763 \\
\hline & Rice & 67,750 & 204,557 & & Peaches & 13,598 & 11,268 \\
\hline & Other Hay & 34,281 & 68,562 & & Raspberries & 3,804 & 3,942 \\
\hline & Sod & 605 & 864 & & Cherries & 2,786 & 2,856 \\
\hline & & & & & Nectarines & 1,161 & 1,430 \\
\hline \multirow[t]{2}{*}{ Tridiphane } & Corn & 255,871 & 440,172 & & Plums & 844 & 1,305 \\
\hline & & & & & Apricots & 828 & 1,194 \\
\hline \multirow[t]{12}{*}{ Trifluralin } & Soybeans & $11,099,394$ & $13,354,394$ & & Onions & 622 & 658 \\
\hline & Cotton & $4,736,822$ & $6,380,474$ & & & & \\
\hline & Alfalfa Hay & 991,611 & 689,449 & Ziram & Almonds & $1,460,271$ & 268,926 \\
\hline & Sunflowers & 645,436 & 840,637 & & Apples & 679,782 & 99,234 \\
\hline & Wheat for Grain & 558,665 & $1,517,700$ & & Peaches & 193,831 & 34,695 \\
\hline & Dry Beans & 324,002 & 558,266 & & Pears & 180,525 & 36,572 \\
\hline & Barley & 290,602 & 488,713 & & Pecans & 160,100 & 38,511 \\
\hline & Sugar Cane for Seed & 245,381 & 125,164 & & Nectarines & 39,428 & 7,152 \\
\hline & and Sugar & & & & Apricots & 20,647 & 3,583 \\
\hline & Tomatoes & 126,694 & 164,441 & & Cherries & 12,628 & 2,331 \\
\hline & Green Peas & 96,034 & 179,413 & & Blueberries & 12,601 & 1,526 \\
\hline & & & & & Strawberries & 562 & 187 \\
\hline
\end{tabular}


Table 5. Ranking of pesticides used in the National Water-Quality Assessment Program study units during 1992-1995 [H, herbicide; I, insecticide; F, fumigant; OP, other pesticide; lb a.i., pounds active ingredient]

\begin{tabular}{|c|c|c|c|c|c|c|c|c|}
\hline Compound & $\begin{array}{c}\text { Pesticide } \\
\text { type }\end{array}$ & $\begin{array}{c}\text { Average } \\
\text { amount } \\
\text { applied } \\
\text { (lb a.i.) }\end{array}$ & $\begin{array}{l}\text { Study unit } \\
\text { area treated } \\
\text { (acres) }\end{array}$ & $\begin{array}{l}\text { Percentage of } \\
\text { national use }\end{array}$ & $\begin{array}{l}\text { Percentage of } \\
\text { U.S. area } \\
\text { treated }\end{array}$ & $\begin{array}{c}\text { Average } \\
\text { amount } \\
\text { applied to } \\
\text { cropland and } \\
\text { pasture area } \\
\text { (lb a.i.) }\end{array}$ & $\begin{array}{l}\text { Percentage of } \\
\text { all reported } \\
\text { pesticides } \\
\text { used in the } \\
\text { study unit }\end{array}$ & $\begin{array}{r}\text { Percent } \\
\text { total } \\
\text { among } \\
\text { uni }\end{array}$ \\
\hline \multicolumn{9}{|c|}{ Acadian-Pontchartrain (ACAD) } \\
\hline \multicolumn{9}{|c|}{ [Study Unit Area: 16,497,232 acres; Area in Cropland and Pasture: $2,356,012$ acres] } \\
\hline Atrazine & $\mathrm{H}$ & 890,515 & 687,191 & 1.39 & 1.21 & 0.38 & 13.82 & 3.17 \\
\hline 2,4-D & $\mathrm{H}$ & 881,177 & $1,344,789$ & 2.37 & 1.73 & 0.37 & 13.68 & 6.56 \\
\hline Propanil & $\mathrm{H}$ & 851,492 & 272,914 & 11.78 & 11.67 & 0.36 & 13.22 & 14.65 \\
\hline Molinate & $\mathrm{H}$ & 820,484 & 249,387 & 16.73 & 17.56 & 0.35 & 12.73 & 20.2 \\
\hline Pendimethalin & $\mathrm{H}$ & 394,383 & 213,675 & 1.9 & 0.99 & 0.17 & 6.12 & 3.68 \\
\hline Trifluralin & $\mathrm{H}$ & 360,805 & 251,462 & 1.82 & 0.99 & 0.15 & 5.6 & 3.68 \\
\hline Methyl parathion & I & 252,351 & 297,244 & 2.83 & 3.97 & 0.11 & 3.92 & 4.55 \\
\hline Metolachlor & $\mathrm{H}$ & 233,960 & 129,641 & 0.4 & 0.42 & 0.1 & 3.63 & 0.86 \\
\hline Alachlor & $\mathrm{H}$ & 137,974 & 119,939 & 0.54 & 0.83 & 0.06 & 2.14 & 1.21 \\
\hline Azinphos methyl & I & 132,399 & 176,228 & 5.31 & 6.82 & 0.06 & 2.06 & 9.47 \\
\hline Carbofuran & I & 123,907 & 193,842 & 2.42 & 2.98 & 0.05 & 1.92 & 5.47 \\
\hline Asulam & $\mathrm{H}$ & 112,458 & 51,824 & 15.6 & 15.51 & 0.05 & 1.75 & 15.75 \\
\hline Metribuzin & $\mathrm{H}$ & 91,409 & 179,241 & 3.38 & 2.13 & 0.04 & 1.42 & 5.53 \\
\hline Glyphosate & $\mathrm{H}$ & 79,100 & 143,329 & 0.49 & 0.6 & 0.03 & 1.23 & 0.95 \\
\hline
\end{tabular}

Apalachicola-Chattahoochee-Flint River Basin (ACFB)

[Study Unit Area: 12,906,062 acres; Area in Cropland and Pasture: 1,701,090 acres]

\begin{tabular}{|c|c|c|c|c|c|c|c|c|}
\hline Chlorothalonil & $\mathrm{F}$ & $2,307,838$ & 428,626 & 18.03 & 13.66 & 1.36 & 18.14 & 25.55 \\
\hline $1,3-\mathrm{D}$ & $\mathrm{OP}$ & $2,045,959$ & 42,615 & 5.11 & 8.65 & 1.2 & 16.08 & 8.33 \\
\hline Sulfur & $\mathrm{F}$ & $1,216,482$ & 166,373 & 1.4 & 6.73 & 0.72 & 9.56 & 2 \\
\hline Methyl bromide & $\mathrm{OP}$ & 844,656 & 3,772 & 2.15 & 1.85 & 0.5 & 6.64 & 3.01 \\
\hline Aldicarb & I & 540,229 & 387,259 & 12.61 & 8.97 & 0.32 & 4.25 & 17 \\
\hline Chlorpyrifos & I & 443,325 & 315,581 & 2.88 & 2.26 & 0.26 & 3.48 & 5.51 \\
\hline Metolachlor & $\mathrm{H}$ & 341,562 & 179,486 & 0.59 & 0.57 & 0.2 & 2.68 & 1.26 \\
\hline Atrazine & $\mathrm{H}$ & 310,675 & 201,177 & 0.49 & 0.35 & 0.18 & 2.44 & 1.11 \\
\hline MSMA & $\mathrm{H}$ & 297,927 & 139,555 & 5.17 & 3.67 & 0.18 & 2.34 & 6.36 \\
\hline Bentazon & $\mathrm{H}$ & 283,109 & 377,647 & 4.21 & 3.77 & 0.17 & 2.22 & 7.29 \\
\hline Pendimethalin & $\mathrm{H}$ & 267,082 & 282,061 & 1.29 & 1.31 & 0.16 & 2.1 & 2.49 \\
\hline $2,4-\mathrm{D}$ & $\mathrm{H}$ & 161,826 & 296,613 & 0.44 & 0.38 & 0.1 & 1.27 & 1.21 \\
\hline Ethalfluralin & $\mathrm{H}$ & 159,530 & 217,612 & 6.49 & 7.06 & 0.09 & 1.25 & 11.08 \\
\hline Carbaryl & I & 155,994 & 83,681 & 3.43 & 2.83 & 0.09 & 1.23 & 6.68 \\
\hline PCNB & $\mathrm{F}$ & 146,795 & 94,948 & 8.66 & 5.99 & 0.09 & 1.15 & 11.98 \\
\hline Vernolate & $\mathrm{H}$ & 139,714 & 78,961 & 28.02 & 30.64 & 0.08 & 1.1 & 32.74 \\
\hline Ethephon & OP & 135,463 & 90,895 & 4.4 & 3.09 & 0.08 & 1.06 & 5.77 \\
\hline Metam sodium & OP & 133,937 & 4,212 & 0.42 & 1.1 & 0.08 & 1.05 & 0.55 \\
\hline
\end{tabular}

Albemarle-Pamlico Drainage (ALBE)

$\begin{array}{lrrrrrrrr}1,3-\mathrm{D} & \text { OP } & 7,837,999 & 116,983 & 19.58 & 23.74 & 2.31 & 34.79 & 31.91 \\ \text { Metam sodium } & \text { OP } & 2,922,329 & 91,897 & 9.07 & 23.94 & 0.86 & 12.97 & 11.92 \\ \text { Metolachlor } & \mathrm{H} & 914,403 & 471,278 & 1.58 & 1.51 & 0.27 & 4.06 & 3.36 \\ \text { Chlorpyrifos } & \mathrm{I} & 693,138 & 334,070 & 4.5 & 2.39 & 0.2 & 3.08\end{array}$


Table 5. Ranking of pesticides used in the National W ater-Quality Assessment Program study units during 1992-1995- Continued

\begin{tabular}{|c|c|c|c|c|c|c|c|c|}
\hline Compound & $\begin{array}{c}\text { Pesticide } \\
\text { type }\end{array}$ & $\begin{array}{l}\text { Average } \\
\text { amount } \\
\text { applied } \\
\text { (lb a.i.) }\end{array}$ & $\begin{array}{l}\text { Study unit } \\
\text { area treated } \\
\text { (acres) }\end{array}$ & $\begin{array}{l}\text { Percentage of } \\
\text { national use }\end{array}$ & $\begin{array}{l}\text { Percentage of } \\
\text { U.S. area } \\
\text { treated }\end{array}$ & $\begin{array}{c}\text { Average } \\
\text { amount } \\
\text { applied to } \\
\text { cropland and } \\
\text { pasture area } \\
\text { (lb a.i.) }\end{array}$ & $\begin{array}{l}\text { Percentage of } \\
\text { all reported } \\
\text { pesticides } \\
\text { used in the } \\
\text { study unit }\end{array}$ & $\begin{array}{c}\text { Percentage of } \\
\text { total use } \\
\text { among study } \\
\text { units }\end{array}$ \\
\hline \multicolumn{9}{|c|}{ Albemarle-Pamlico Drainage (ALBE)—Continued } \\
\hline Alachlor & $\mathrm{H}$ & 595,398 & 376,662 & 2.32 & 2.59 & 0.18 & 2.64 & 5.21 \\
\hline Chlorothalonil & $\mathrm{F}$ & 537,828 & 213,284 & 4.2 & 6.8 & 0.16 & 2.39 & 5.95 \\
\hline Acephate & I & 526,309 & 328,322 & 14.46 & 10.3 & 0.15 & 2.34 & 25.5 \\
\hline Maleic hydrazide & $\mathrm{OP}$ & 493,458 & 159,696 & 23.22 & 22.14 & 0.15 & 2.19 & 45.66 \\
\hline Atrazine & $\mathrm{H}$ & 408,459 & 365,854 & 0.64 & 0.64 & 0.12 & 1.81 & 1.46 \\
\hline Aldicarb & $\mathrm{I}$ & 380,977 & 439,612 & 8.9 & 10.19 & 0.11 & 1.69 & 11.99 \\
\hline $2,4-\mathrm{D}$ & $\mathrm{H}$ & 325,241 & 684,729 & 0.87 & 0.88 & 0.1 & 1.44 & 2.42 \\
\hline Pendimethalin & $\mathrm{H}$ & 314,730 & 407,128 & 1.52 & 1.89 & 0.09 & 1.4 & 2.93 \\
\hline \multicolumn{9}{|c|}{ Allegheny and Monongahela Basins (ALMN) } \\
\hline \multicolumn{9}{|c|}{ [Study Unit Area: 12,219,692 acres; Area in Cropland and Pasture: 1,401,659 acres] } \\
\hline Metolachlor & $\mathrm{H}$ & 236,098 & 131,184 & 0.41 & 0.42 & 0.17 & 16.07 & 0.87 \\
\hline Atrazine & $\mathrm{H}$ & 192,926 & 165,413 & 0.3 & 0.29 & 0.14 & 13.13 & 0.69 \\
\hline Mancozeb & $\mathrm{F}$ & 110,929 & 20,413 & 1.34 & 1 & 0.08 & 7.55 & 2.07 \\
\hline Pendimethalin & $\mathrm{H}$ & 92,184 & 73,576 & 0.45 & 0.34 & 0.07 & 6.28 & 0.86 \\
\hline Glyphosate & $\mathrm{H}$ & 68,533 & 73,400 & 0.43 & 0.31 & 0.05 & 4.67 & 0.82 \\
\hline Alachlor & $\mathrm{H}$ & 63,184 & 36,814 & 0.25 & 0.25 & 0.05 & 4.3 & 0.55 \\
\hline Sulfur & $\mathrm{F}$ & 57,859 & 4,863 & 0.07 & 0.2 & 0.04 & 3.94 & 0.1 \\
\hline Simazine & $\mathrm{H}$ & 55,049 & 59,016 & 1.14 & 1.72 & 0.04 & 3.75 & 1.79 \\
\hline Oil & I & 54,522 & 1,779 & 0.1 & 0.13 & 0.04 & 3.71 & 0.14 \\
\hline Chlorpyrifos & I & 48,032 & 44,386 & 0.31 & 0.32 & 0.03 & 3.27 & 0.6 \\
\hline Cyanazine & $\mathrm{H}$ & 47,927 & 28,324 & 0.16 & 0.18 & 0.03 & 3.26 & 0.35 \\
\hline Dimethoate & I & 42,906 & 70,582 & 1.26 & 1.37 & 0.03 & 2.92 & 3.88 \\
\hline Carbaryl & I & 35,493 & 12,961 & 0.78 & 0.44 & 0.03 & 2.42 & 1.52 \\
\hline $2,4-\mathrm{D}$ & $\mathrm{H}$ & 33,157 & 67,782 & 0.09 & 0.09 & 0.02 & 2.26 & 0.25 \\
\hline Captan & $\mathrm{F}$ & 26,647 & 4,759 & 0.7 & 0.81 & 0.02 & 1.81 & 1.37 \\
\hline Carbofuran & I & 23,425 & 33,608 & 0.46 & 0.52 & 0.02 & 1.59 & 1.03 \\
\hline Ferbam & $\mathrm{F}$ & 17,885 & 6,518 & 7.99 & 9.29 & 0.01 & 1.22 & 13.51 \\
\hline Terbufos & I & 17,288 & 12,887 & 0.24 & 0.19 & 0.01 & 1.18 & 0.56 \\
\hline Chlorothalonil & $\mathrm{F}$ & 15,799 & 4,592 & 0.12 & 0.15 & 0.01 & 1.08 & 0.18 \\
\hline Dicamba & $\mathrm{H}$ & 15,731 & 53,532 & 0.16 & 0.14 & 0.01 & 1.07 & 0.39 \\
\hline \multicolumn{9}{|c|}{ Central Arizona Basins (CAZB) } \\
\hline \multicolumn{9}{|c|}{ [Study Unit Area: 21,537,788 acres; Area in Cropland and Pasture: 448,332 acres] } \\
\hline Sodium chlorate & OP & 621,644 & 108,489 & 11.86 & 6.34 & 1.39 & 16.01 & 15.35 \\
\hline $1,3-\mathrm{D}$ & OP & 444,830 & 13,413 & 1.11 & 2.72 & 0.99 & 11.46 & 1.81 \\
\hline Acephate & I & 307,803 & 235,078 & 8.46 & 7.38 & 0.69 & 7.93 & 14.91 \\
\hline Chlorpyrifos & I & 237,618 & 169,179 & 1.54 & 1.21 & 0.53 & 6.12 & 2.95 \\
\hline $2,4-\mathrm{D}$ & $\mathrm{H}$ & 232,258 & 462,976 & 0.62 & 0.6 & 0.52 & 5.98 & 1.73 \\
\hline Endosulfan & I & 148,103 & 105,008 & 7.09 & 6.5 & 0.33 & 3.81 & 10.97 \\
\hline Sulfur & $\mathrm{F}$ & 129,506 & 6,999 & 0.15 & 0.28 & 0.29 & 3.34 & 0.21 \\
\hline Azinphos methyl & I & 112,953 & 31,562 & 4.53 & 1.22 & 0.25 & 2.91 & 8.08 \\
\hline Trifluralin & $\mathrm{H}$ & 105,992 & 105,436 & 0.54 & 0.42 & 0.24 & 2.73 & 1.08 \\
\hline
\end{tabular}


Table 5. Ranking of pesticides used in the National W ater-Quality Assessment Program study units during 1992-1995- Continued

\begin{tabular}{|c|c|c|c|c|c|c|c|c|}
\hline Compound & $\begin{array}{c}\text { Pesticide } \\
\text { type }\end{array}$ & $\begin{array}{l}\text { Average } \\
\text { amount } \\
\text { applied } \\
\text { (lb a.i.) }\end{array}$ & $\begin{array}{l}\text { Study unit } \\
\text { area treated } \\
\text { (acres) }\end{array}$ & $\begin{array}{l}\text { Percentage of } \\
\text { national use }\end{array}$ & $\begin{array}{c}\text { Percentage of } \\
\text { U.S. area } \\
\text { treated }\end{array}$ & $\begin{array}{c}\text { Average } \\
\text { amount } \\
\text { applied to } \\
\text { cropland and } \\
\text { pasture area } \\
\text { (lb a.i.) }\end{array}$ & $\begin{array}{l}\text { Percentage of } \\
\text { all reported } \\
\text { pesticides } \\
\text { used in the } \\
\text { study unit }\end{array}$ & $\begin{array}{c}\text { Percentage of } \\
\text { total use } \\
\text { among study } \\
\text { units }\end{array}$ \\
\hline \multicolumn{9}{|c|}{ Central Arizona Basins (CAZB)—Continued } \\
\hline Profenofos & I & 102,459 & 78,213 & 4.83 & 4.61 & 0.23 & 2.64 & 7.01 \\
\hline Tribufos & $\mathrm{OP}$ & 100,668 & 95,874 & 2.71 & 2.41 & 0.22 & 2.59 & 3.79 \\
\hline Propargite & I & 96,354 & 47,937 & 2.81 & 2.47 & 0.21 & 2.48 & 4.47 \\
\hline Prometryn & $\mathrm{H}$ & 87,195 & 80,736 & 4.73 & 3.16 & 0.19 & 2.25 & 8.46 \\
\hline Dicofol & I & 86,642 & 77,524 & 7.95 & 9.27 & 0.19 & 2.23 & 10.25 \\
\hline Fenpropathrin & I & 81,499 & 171,427 & 55.55 & 53.53 & 0.18 & 2.1 & 94.8 \\
\hline Malathion & I & 75,128 & 38,124 & 2.42 & 1.97 & 0.17 & 1.94 & 5.21 \\
\hline Diuron & $\mathrm{H}$ & 63,849 & 130,187 & 1.7 & 4.01 & 0.14 & 1.64 & 2.36 \\
\hline Thidiazuron & OP & 62,520 & 148,857 & 13.82 & 5.48 & 0.14 & 1.61 & 22.16 \\
\hline Methyl parathion & I & 57,984 & 38,610 & 0.65 & 0.52 & 0.13 & 1.49 & 1.05 \\
\hline Methomyl & I & 56,232 & 72,093 & 2.3 & 2.11 & 0.13 & 1.45 & 3.86 \\
\hline Pendimethalin & $\mathrm{H}$ & 50,511 & 65,598 & 0.24 & 0.31 & 0.11 & 1.3 & 0.47 \\
\hline Aldicarb & I & 49,199 & 63,075 & 1.15 & 1.46 & 0.11 & 1.27 & 1.55 \\
\hline Glyphosate & $\mathrm{H}$ & 44,723 & 33,738 & 0.28 & 0.14 & 0.1 & 1.15 & 0.54 \\
\hline \multicolumn{9}{|c|}{ Central Columbia Plateau (CCPT) } \\
\hline \multicolumn{9}{|c|}{ [Study Unit Area: 8,371,111 acres; Area in Cropland and Pasture: 2,922,874 acres] } \\
\hline Metam sodium & $\mathrm{OP}$ & $7,090,563$ & 45,881 & 22 & 11.95 & 2.43 & 38.63 & 28.93 \\
\hline $1,3-\mathrm{D}$ & $\mathrm{OP}$ & $3,126,158$ & 19,058 & 7.81 & 3.87 & 1.07 & 17.03 & 12.73 \\
\hline Oil & I & $1,732,642$ & 46,091 & 3.26 & 3.4 & 0.59 & 9.44 & 4.49 \\
\hline Sulfuric acid & $\mathrm{OP}$ & $1,341,165$ & 6,534 & 5.17 & 6.19 & 0.46 & 7.31 & 6.17 \\
\hline $2,4-\mathrm{D}$ & $\mathrm{H}$ & 526,520 & 991,350 & 1.41 & 1.27 & 0.18 & 2.87 & 3.92 \\
\hline EPTC & $\mathrm{H}$ & 326,598 & 101,251 & 2.32 & 2.52 & 0.11 & 1.78 & 4.95 \\
\hline Diuron & $\mathrm{H}$ & 314,555 & 261,189 & 8.4 & 8.04 & 0.11 & 1.71 & 11.62 \\
\hline Chlorothalonil & $\mathrm{F}$ & 284,512 & 70,275 & 2.22 & 2.24 & 0.1 & 1.55 & 3.15 \\
\hline Sulfur & $\mathrm{F}$ & 259,228 & 35,576 & 0.3 & 1.44 & 0.09 & 1.41 & 0.43 \\
\hline MCPA & $\mathrm{H}$ & 243,746 & 608,257 & 5.23 & 4.94 & 0.08 & 1.33 & 10.81 \\
\hline Chloropicrin & $\mathrm{OP}$ & 242,113 & 4,901 & 3.25 & 4 & 0.08 & 1.32 & 5.63 \\
\hline Triallate & $\mathrm{H}$ & 190,130 & 159,891 & 8.33 & 7.19 & 0.07 & 1.04 & 18.99 \\
\hline \multicolumn{9}{|c|}{ Cheyenne and Belle Fourche Basins (CHEY) } \\
\hline \multicolumn{9}{|c|}{ [Study Unit Area: $15,491,569$ acres; Area in Cropland and Pasture: $1,015,577$ acres] } \\
\hline $2,4-\mathrm{D}$ & $\mathrm{H}$ & 329,605 & 470,723 & 0.89 & 0.61 & 0.32 & 54 & 2.45 \\
\hline Dicamba & $\mathrm{H}$ & 50,009 & 182,944 & 0.52 & 0.47 & 0.05 & 8.19 & 1.23 \\
\hline Picloram & $\mathrm{H}$ & 40,458 & 186,204 & 2.35 & 2.62 & 0.04 & 6.63 & 11.55 \\
\hline $1,3-\mathrm{D}$ & $\mathrm{OP}$ & 22,342 & 155 & 0.06 & 0.03 & 0.02 & 3.66 & 0.09 \\
\hline Carbaryl & I & 18,644 & 8,296 & 0.41 & 0.28 & 0.02 & 3.05 & 0.8 \\
\hline MCPA & $\mathrm{H}$ & 17,591 & 46,689 & 0.38 & 0.38 & 0.02 & 2.88 & 0.78 \\
\hline EPTC & $\mathrm{H}$ & 17,178 & 5,671 & 0.12 & 0.14 & 0.02 & 2.81 & 0.26 \\
\hline Glyphosate & $\mathrm{H}$ & 9,675 & 30,337 & 0.06 & 0.13 & 0.01 & 1.58 & 0.12 \\
\hline Ethyl parathion & I & 8,842 & 25,148 & 0.43 & 0.74 & 0.01 & 1.45 & 1.64 \\
\hline Chlorpyrifos & I & 7,271 & 10,351 & 0.05 & 0.07 & 0.01 & 1.19 & 0.09 \\
\hline Trifluralin & $\mathrm{H}$ & 7,268 & 9,265 & 0.04 & 0.04 & 0.01 & 1.19 & 0.07 \\
\hline Alachlor & $\mathrm{H}$ & 7,191 & 4,020 & 0.03 & 0.03 & 0.01 & 1.18 & 0.06 \\
\hline
\end{tabular}


Table 5. Ranking of pesticides used in the National W ater-Quality Assessment Program study units during 1992-1995- Continued

\begin{tabular}{|c|c|c|c|c|c|c|c|c|}
\hline Compound & $\begin{array}{c}\text { Pesticide } \\
\text { type }\end{array}$ & $\begin{array}{l}\text { Average } \\
\text { amount } \\
\text { applied } \\
\text { (lb a.i.) }\end{array}$ & $\begin{array}{l}\text { Study unit } \\
\text { area treated } \\
\text { (acres) }\end{array}$ & $\begin{array}{l}\text { Percentage of } \\
\text { national use }\end{array}$ & $\begin{array}{l}\text { Percentage of } \\
\text { U.S. area } \\
\text { treated }\end{array}$ & $\begin{array}{c}\text { Average } \\
\text { amount } \\
\text { applied to } \\
\text { cropland and } \\
\text { pasture area } \\
\text { (lb a.i.) }\end{array}$ & $\begin{array}{l}\text { Percentage of } \\
\text { all reported } \\
\text { pesticides } \\
\text { used in the } \\
\text { study unit }\end{array}$ & $\begin{array}{c}\text { Percentage of } \\
\text { total use } \\
\text { among study } \\
\text { units }\end{array}$ \\
\hline \multicolumn{9}{|c|}{ Cheyenne and Belle Fourche Basins (CHEY)—Continued } \\
\hline Metolachlor & $\mathrm{H}$ & 6,731 & 3,566 & 0.01 & 0.01 & 0.01 & 1.1 & 0.03 \\
\hline Atrazine & $\mathrm{H}$ & 6,572 & 7,689 & 0.01 & 0.01 & 0.01 & 1.08 & 0.02 \\
\hline 2,4-Db & $\mathrm{H}$ & 6,227 & 12,455 & 0.65 & 0.28 & 0.01 & 1.02 & 1.22 \\
\hline Acetochlor & $\mathrm{H}$ & 6,154 & 3,626 & 0.03 & 0.03 & 0.01 & 1.01 & 0.06 \\
\hline \multicolumn{9}{|c|}{ Central Nebraska Basins (CNBR) } \\
\hline \multicolumn{9}{|c|}{ [Study Unit Area: 19,462,958 acres; Area in Cropland and Pasture: 7,822,815 acres] } \\
\hline Atrazine & $\mathrm{H}$ & $3,453,670$ & $3,057,287$ & 5.4 & 5.37 & 0.44 & 25.6 & 12.3 \\
\hline Metolachlor & $\mathrm{H}$ & $2,253,285$ & $1,441,447$ & 3.89 & 4.61 & 0.29 & 16.71 & 8.28 \\
\hline Cyanazine & $\mathrm{H}$ & $1,147,039$ & 709,493 & 3.88 & 4.48 & 0.15 & 8.5 & 8.28 \\
\hline Alachlor & $\mathrm{H}$ & $1,117,741$ & 876,392 & 4.36 & 6.03 & 0.14 & 8.29 & 9.79 \\
\hline Acetochlor & $\mathrm{H}$ & 749,404 & 407,284 & 3.14 & 3.44 & 0.1 & 5.56 & 6.76 \\
\hline $2,4-\mathrm{D}$ & $\mathrm{H}$ & 602,727 & $1,144,065$ & 1.62 & 1.47 & 0.08 & 4.47 & 4.49 \\
\hline Terbufos & I & 396,985 & 410,285 & 5.51 & 6.15 & 0.05 & 2.94 & 12.87 \\
\hline Chlorpyrifos & I & 389,052 & 389,853 & 2.53 & 2.79 & 0.05 & 2.88 & 4.83 \\
\hline Methyl parathion & I & 371,468 & 629,571 & 4.16 & 8.4 & 0.05 & 2.75 & 6.7 \\
\hline Butylate & $\mathrm{H}$ & 337,673 & 74,051 & 4.39 & 3.73 & 0.04 & 2.5 & 11.35 \\
\hline Trifluralin & $\mathrm{H}$ & 311,074 & 412,173 & 1.57 & 1.63 & 0.04 & 2.31 & 3.17 \\
\hline Propachlor & $\mathrm{H}$ & 251,745 & 109,092 & 6.51 & 6.51 & 0.03 & 1.87 & 19.13 \\
\hline Pendimethalin & $\mathrm{H}$ & 246,841 & 297,405 & 1.19 & 1.38 & 0.03 & 1.83 & 2.3 \\
\hline Fonofos & I & 208,824 & 222,153 & 7.43 & 9.19 & 0.03 & 1.55 & 16.48 \\
\hline Dicamba & $\mathrm{H}$ & 184,360 & 623,207 & 1.92 & 1.6 & 0.02 & 1.37 & 4.53 \\
\hline EPTC & $\mathrm{H}$ & 165,132 & 45,651 & 1.17 & 1.14 & 0.02 & 1.22 & 2.5 \\
\hline Carbofuran & I & 156,616 & 195,191 & 3.06 & 3 & 0.02 & 1.16 & 6.92 \\
\hline Carbaryl & I & 140,812 & 109,468 & 3.09 & 3.71 & 0.02 & 1.04 & 6.03 \\
\hline \multicolumn{9}{|c|}{ Connecticut, Housatonic, and Thames River Basins (CONN) } \\
\hline \multicolumn{9}{|c|}{ [Study Unit Area: 10,037,989 acres; Area in Cropland and Pasture: 508,084 acres] } \\
\hline Oil & I & 228,626 & 7,827 & 0.43 & 0.58 & 0.45 & 24.24 & 0.59 \\
\hline Metolachlor & $\mathrm{H}$ & 119,622 & 62,762 & 0.21 & 0.2 & 0.24 & 12.68 & 0.44 \\
\hline Atrazine & $\mathrm{H}$ & 115,399 & 80,903 & 0.18 & 0.14 & 0.23 & 12.24 & 0.41 \\
\hline Chlorothalonil & $\mathrm{F}$ & 56,632 & 7,547 & 0.44 & 0.24 & 0.11 & 6.01 & 0.63 \\
\hline Captan & $\mathrm{F}$ & 50,092 & 7,073 & 1.32 & 1.21 & 0.1 & 5.31 & 2.57 \\
\hline Mancozeb & $\mathrm{F}$ & 48,867 & 9,762 & 0.59 & 0.48 & 0.1 & 5.18 & 0.91 \\
\hline Alachlor & $\mathrm{H}$ & 23,773 & 12,394 & 0.09 & 0.09 & 0.05 & 2.52 & 0.21 \\
\hline Cyanazine & $\mathrm{H}$ & 22,848 & 14,450 & 0.08 & 0.09 & 0.04 & 2.42 & 0.17 \\
\hline $2,4-\mathrm{D}$ & $\mathrm{H}$ & 21,403 & 52,724 & 0.06 & 0.07 & 0.04 & 2.27 & 0.16 \\
\hline Azinphos methyl & I & 19,481 & 8,395 & 0.78 & 0.33 & 0.04 & 2.07 & 1.39 \\
\hline Cryolite & I & 19,120 & 1,202 & 0.62 & 0.25 & 0.04 & 2.03 & 0.9 \\
\hline Metiram & $\mathrm{F}$ & 16,979 & 1,061 & 1.84 & 0.84 & 0.03 & 1.8 & 4.03 \\
\hline Methyl bromide & OP & 16,583 & 98 & 0.04 & 0.05 & 0.03 & 1.76 & 0.06 \\
\hline Thiram & $\mathrm{F}$ & 15,526 & 3,093 & 5.83 & 6.66 & 0.03 & 1.65 & 12.46 \\
\hline
\end{tabular}


Table 5. Ranking of pesticides used in the National W ater-Quality Assessment Program study units during 1992-1995- Continued

\begin{tabular}{|c|c|c|c|c|c|c|c|c|}
\hline Compound & $\begin{array}{c}\text { Pesticide } \\
\text { type }\end{array}$ & $\begin{array}{l}\text { Average } \\
\text { amount } \\
\text { applied } \\
\text { (lb a.i.) }\end{array}$ & $\begin{array}{l}\text { Study unit } \\
\text { area treated } \\
\text { (acres) }\end{array}$ & $\begin{array}{l}\text { Percentage of } \\
\text { national use }\end{array}$ & $\begin{array}{l}\text { Percentage of } \\
\text { U.S. area } \\
\text { treated }\end{array}$ & $\begin{array}{c}\text { Average } \\
\text { amount } \\
\text { applied to } \\
\text { cropland and } \\
\text { pasture area } \\
\text { (lb a.i.) } \\
\end{array}$ & $\begin{array}{l}\text { Percentage of } \\
\text { all reported } \\
\text { pesticides } \\
\text { used in the } \\
\text { study unit }\end{array}$ & $\begin{array}{l}\text { Percentage of } \\
\text { total use } \\
\text { among study } \\
\text { units }\end{array}$ \\
\hline \multicolumn{9}{|c|}{ Connecticut, Housatonic, and Thames River Basins (CONN)—Continued } \\
\hline Chlorpyrifos & I & 12,165 & 11,863 & 0.08 & 0.09 & 0.02 & 1.29 & 0.15 \\
\hline Carbofuran & I & 11,299 & 10,252 & 0.22 & 0.16 & 0.02 & 1.2 & 0.5 \\
\hline Dicamba & $\mathrm{H}$ & 10,583 & 24,640 & 0.11 & 0.06 & 0.02 & 1.12 & 0.26 \\
\hline \multicolumn{9}{|c|}{ Delaware River Basin (DELR) } \\
\hline \multicolumn{9}{|c|}{ [Study Unit Area: 7,728,528 acres; Area in Cropland and Pasture: 1,116,698 acres] } \\
\hline Metolachlor & $\mathrm{H}$ & 512,258 & 281,033 & 0.88 & 0.9 & 0.46 & 17.04 & 1.88 \\
\hline Sulfur & $\mathrm{F}$ & 505,202 & 8,909 & 0.58 & 0.36 & 0.45 & 16.81 & 0.83 \\
\hline Atrazine & $\mathrm{H}$ & 308,352 & 256,144 & 0.48 & 0.45 & 0.28 & 10.26 & 1.1 \\
\hline Oil & I & 199,546 & 7,297 & 0.38 & 0.54 & 0.18 & 6.64 & 0.52 \\
\hline Alachlor & $\mathrm{H}$ & 127,328 & 70,547 & 0.5 & 0.49 & 0.11 & 4.24 & 1.12 \\
\hline Captan & $\mathrm{F}$ & 108,133 & 14,995 & 2.84 & 2.56 & 0.1 & 3.6 & 5.55 \\
\hline Pendimethalin & $\mathrm{H}$ & 101,350 & 87,112 & 0.49 & 0.41 & 0.09 & 3.37 & 0.95 \\
\hline Mancozeb & $\mathrm{F}$ & 89,411 & 16,797 & 1.08 & 0.82 & 0.08 & 2.98 & 1.67 \\
\hline Glyphosate & $\mathrm{H}$ & 75,848 & 68,312 & 0.47 & 0.29 & 0.07 & 2.52 & 0.91 \\
\hline Cyanazine & $\mathrm{H}$ & 70,884 & 45,259 & 0.24 & 0.29 & 0.06 & 2.36 & 0.51 \\
\hline Chlorothalonil & $\mathrm{F}$ & 70,219 & 14,752 & 0.55 & 0.47 & 0.06 & 2.34 & 0.78 \\
\hline $2,4-\mathrm{D}$ & $\mathrm{H}$ & 56,915 & 117,026 & 0.15 & 0.15 & 0.05 & 1.89 & 0.42 \\
\hline Chlorpyrifos & I & 56,914 & 49,102 & 0.37 & 0.35 & 0.05 & 1.89 & 0.71 \\
\hline Cryolite & I & 45,256 & 2,982 & 1.47 & 0.62 & 0.04 & 1.51 & 2.14 \\
\hline Linuron & $\mathrm{H}$ & 44,663 & 87,343 & 2.26 & 2.77 & 0.04 & 1.49 & 4.13 \\
\hline Simazine & $\mathrm{H}$ & 36,890 & 36,792 & 0.77 & 1.07 & 0.03 & 1.23 & 1.2 \\
\hline Maneb & $\mathrm{F}$ & 34,248 & 9,564 & 1.14 & 1.36 & 0.03 & 1.14 & 1.99 \\
\hline Methomyl & I & 32,115 & 23,438 & 1.31 & 0.69 & 0.03 & 1.07 & 2.2 \\
\hline \multicolumn{9}{|c|}{ Delmarva Peninsula (DLMV) } \\
\hline \multicolumn{9}{|c|}{ [Study Unit Area: 3,608,286 acres; Area in Cropland and Pasture: $1,307,479$ acres] } \\
\hline Metolachlor & $\mathrm{H}$ & 951,197 & 516,034 & 1.64 & 1.65 & 0.73 & 25.74 & 3.5 \\
\hline Atrazine & $\mathrm{H}$ & 504,176 & 335,746 & 0.79 & 0.59 & 0.39 & 13.64 & 1.8 \\
\hline Alachlor & $\mathrm{H}$ & 341,809 & 191,021 & 1.33 & 1.32 & 0.26 & 9.25 & 2.99 \\
\hline Mancozeb & $\mathrm{F}$ & 170,242 & 25,860 & 2.05 & 1.27 & 0.13 & 4.61 & 3.17 \\
\hline Linuron & $\mathrm{H}$ & 146,078 & 302,610 & 7.4 & 9.61 & 0.11 & 3.95 & 13.49 \\
\hline Chlorothalonil & $\mathrm{F}$ & 135,021 & 22,308 & 1.06 & 0.71 & 0.1 & 3.65 & 1.5 \\
\hline Simazine & $\mathrm{H}$ & 133,735 & 133,959 & 2.78 & 3.9 & 0.1 & 3.62 & 4.36 \\
\hline Glyphosate & $\mathrm{H}$ & 107,393 & 93,857 & 0.67 & 0.39 & 0.08 & 2.91 & 1.29 \\
\hline Paraquat & $\mathrm{H}$ & 94,421 & 226,578 & 2.23 & 2.39 & 0.07 & 2.55 & 4.01 \\
\hline $2,4-\mathrm{D}$ & $\mathrm{H}$ & 88,555 & 234,385 & 0.24 & 0.3 & 0.07 & 2.4 & 0.66 \\
\hline Cyanazine & $\mathrm{H}$ & 77,269 & 53,131 & 0.26 & 0.34 & 0.06 & 2.09 & 0.56 \\
\hline Maneb & $\mathrm{F}$ & 68,021 & 15,836 & 2.26 & 2.25 & 0.05 & 1.84 & 3.96 \\
\hline Chlorpyrifos & I & 66,531 & 62,379 & 0.43 & 0.45 & 0.05 & 1.8 & 0.83 \\
\hline Cryolite & I & 66,407 & 4,328 & 2.16 & 0.9 & 0.05 & 1.8 & 3.14 \\
\hline Methyl bromide & OP & 64,435 & 730 & 0.16 & 0.36 & 0.05 & 1.74 & 0.23 \\
\hline
\end{tabular}


Table 5. Ranking of pesticides used in the National W ater-Quality Assessment Program study units during 1992-1995- Continued

\begin{tabular}{|c|c|c|c|c|c|c|c|c|}
\hline Compound & $\begin{array}{l}\text { Pesticide } \\
\text { type }\end{array}$ & $\begin{array}{l}\text { Average } \\
\text { amount } \\
\text { applied } \\
\text { (lb a.i.) }\end{array}$ & $\begin{array}{l}\text { Study unit } \\
\text { area treated } \\
\text { (acres) }\end{array}$ & $\begin{array}{c}\text { Percentage of } \\
\text { national use }\end{array}$ & $\begin{array}{c}\text { Percentage of } \\
\text { U.S. area } \\
\text { treated }\end{array}$ & $\begin{array}{c}\text { Average } \\
\text { amount } \\
\text { applied to } \\
\text { cropland and } \\
\text { pasture area } \\
\text { (lb a.i.) }\end{array}$ & $\begin{array}{l}\text { Percentage of } \\
\text { all reported } \\
\text { pesticides } \\
\text { used in the } \\
\text { study unit }\end{array}$ & $\begin{array}{c}\text { Percentage of } \\
\text { total use } \\
\text { among study } \\
\text { units }\end{array}$ \\
\hline \multicolumn{9}{|c|}{ Delmarva Peninsula (DLMV)—Continued } \\
\hline Pendimethalin & $\mathrm{H}$ & 63,736 & 81,739 & 0.31 & 0.38 & 0.05 & 1.72 & 0.59 \\
\hline Carbofuran & I & 52,246 & 42,849 & 1.02 & 0.66 & 0.04 & 1.41 & 2.31 \\
\hline Terbufos & I & 44,831 & 44,428 & 0.62 & 0.67 & 0.03 & 1.21 & 1.45 \\
\hline
\end{tabular}

Eastern Iowa Basins (EIWA)

\begin{tabular}{|c|c|c|c|c|c|c|c|c|}
\hline Metolachlor & $\mathrm{H}$ & $3,557,616$ & $1,669,292$ & 6.14 & 5.34 & 0.4 & 19.3 & 13.07 \\
\hline Atrazine & $\mathrm{H}$ & $2,660,692$ & $2,968,923$ & 4.16 & 5.21 & 0.3 & 14.44 & 9.48 \\
\hline Acetochlor & $\mathrm{H}$ & $2,634,229$ & $1,271,458$ & 11.05 & 10.73 & 0.3 & 14.29 & 23.77 \\
\hline Cyanazine & $\mathrm{H}$ & $2,121,908$ & 868,143 & 7.18 & 5.48 & 0.24 & 11.51 & 15.32 \\
\hline Trifluralin & $\mathrm{H}$ & 807,171 & 956,997 & 4.08 & 3.77 & 0.09 & 4.38 & 8.23 \\
\hline Pendimethalin & $\mathrm{H}$ & 758,342 & 762,845 & 3.66 & 3.55 & 0.09 & 4.11 & 7.07 \\
\hline EPTC & $\mathrm{H}$ & 631,126 & 152,967 & 4.48 & 3.81 & 0.07 & 3.42 & 9.57 \\
\hline Dicamba & $\mathrm{H}$ & 591,858 & $1,765,026$ & 6.17 & 4.53 & 0.07 & 3.21 & 14.55 \\
\hline Chlorpyrifos & I & 563,425 & 517,461 & 3.66 & 3.71 & 0.06 & 3.06 & 7 \\
\hline Alachlor & $\mathrm{H}$ & 472,561 & 222,938 & 1.84 & 1.53 & 0.05 & 2.56 & 4.14 \\
\hline $2,4-\mathrm{D}$ & $\mathrm{H}$ & 472,294 & $1,115,737$ & 1.27 & 1.43 & 0.05 & 2.56 & 3.52 \\
\hline Bentazon & $\mathrm{H}$ & 372,886 & 572,714 & 5.54 & 5.72 & 0.04 & 2.02 & 9.6 \\
\hline Terbufos & I & 346,289 & 331,880 & 4.81 & 4.97 & 0.04 & 1.88 & 11.23 \\
\hline Glyphosate & $\mathrm{H}$ & 339,195 & 610,274 & 2.12 & 2.56 & 0.04 & 1.84 & 4.08 \\
\hline Propachlor & $\mathrm{H}$ & 284,103 & 146,835 & 7.35 & 8.77 & 0.03 & 1.54 & 21.58 \\
\hline Dimethenamid & $\mathrm{H}$ & 229,584 & 210,627 & 8.78 & 9.07 & 0.03 & 1.25 & 17.51 \\
\hline Bromoxynil & $\mathrm{H}$ & 203,687 & 817,195 & 6.03 & 6.6 & 0.02 & 1.11 & 14.17 \\
\hline
\end{tabular}

Georgia-Florida Coastal Plain (GAFL)

[Study Unit Area: 37,164,232 acres; Area in Cropland and Pasture: 3,687,851 acres]

\begin{tabular}{|c|c|c|c|c|c|c|c|c|}
\hline Oil & I & $5,615,907$ & 110,986 & 10.56 & 8.19 & 1.52 & 18.01 & 14.54 \\
\hline Methyl bromide & $\mathrm{OP}$ & $4,335,167$ & 21,072 & 11.05 & 10.31 & 1.18 & 13.9 & 15.44 \\
\hline $1,3-\mathrm{D}$ & $\mathrm{OP}$ & $2,499,870$ & 55,733 & 6.25 & 11.31 & 0.68 & 8.02 & 10.18 \\
\hline Sulfur & $\mathrm{F}$ & $2,403,357$ & 184,394 & 2.77 & 7.46 & 0.65 & 7.71 & 3.95 \\
\hline Chlorothalonil & $\mathrm{F}$ & $2,241,644$ & 411,978 & 17.51 & 13.13 & 0.61 & 7.19 & 24.81 \\
\hline Copper & $\mathrm{F}$ & 736,008 & 143,798 & 6.99 & 5.74 & 0.2 & 2.36 & 8.45 \\
\hline Aldicarb & I & 700,620 & 451,885 & 16.36 & 10.47 & 0.19 & 2.25 & 22.04 \\
\hline Chloropicrin & $\mathrm{OP}$ & 679,591 & 11,409 & 9.13 & 9.3 & 0.18 & 2.18 & 15.81 \\
\hline Chlorpyrifos & I & 673,804 & 463,344 & 4.37 & 3.32 & 0.18 & 2.16 & 8.37 \\
\hline Atrazine & $\mathrm{H}$ & 656,797 & 422,398 & 1.03 & 0.74 & 0.18 & 2.11 & 2.34 \\
\hline Mancozeb & $\mathrm{F}$ & 621,122 & 101,059 & 7.48 & 4.95 & 0.17 & 1.99 & 11.58 \\
\hline MSMA & $\mathrm{H}$ & 489,794 & 223,030 & 8.5 & 5.86 & 0.13 & 1.57 & 10.46 \\
\hline Glyphosate & $\mathrm{H}$ & 440,992 & 291,223 & 2.75 & 1.22 & 0.12 & 1.41 & 5.3 \\
\hline Bromacil & $\mathrm{H}$ & 439,790 & 141,412 & 36.81 & 32.13 & 0.12 & 1.41 & 39.87 \\
\hline $2,4-\mathrm{D}$ & $\mathrm{H}$ & 419,889 & 740,818 & 1.13 & 0.95 & 0.11 & 1.35 & 3.13 \\
\hline Diuron & $\mathrm{H}$ & 413,874 & 208,495 & 11.05 & 6.42 & 0.11 & 1.33 & 15.29 \\
\hline Simazine & $\mathrm{H}$ & 375,003 & 104,092 & 7.8 & 3.03 & 0.1 & 1.2 & 12.22 \\
\hline
\end{tabular}


Table 5. Ranking of pesticides used in the National W ater-Quality Assessment Program study units during 1992-1995- Continued

\begin{tabular}{|c|c|c|c|c|c|c|c|c|}
\hline Compound & $\begin{array}{c}\text { Pesticide } \\
\text { type }\end{array}$ & $\begin{array}{l}\text { Average } \\
\text { amount } \\
\text { applied } \\
\text { (lb a.i.) }\end{array}$ & $\begin{array}{l}\text { Study unit } \\
\text { area treated } \\
\text { (acres) }\end{array}$ & $\begin{array}{l}\text { Percentage of } \\
\text { national use }\end{array}$ & $\begin{array}{l}\text { Percentage of } \\
\text { U.S. area } \\
\text { treated }\end{array}$ & $\begin{array}{c}\text { Average } \\
\text { amount } \\
\text { applied to } \\
\text { cropland and } \\
\text { pasture area } \\
\text { (lb a.i.) }\end{array}$ & $\begin{array}{l}\text { Percentage of } \\
\text { all reported } \\
\text { pesticides } \\
\text { used in the } \\
\text { study unit }\end{array}$ & $\begin{array}{c}\text { Percentage of } \\
\text { total use } \\
\text { among study } \\
\text { units }\end{array}$ \\
\hline \multicolumn{9}{|c|}{ Georgia-Florida Coastal Plain (GAFL)—Continued } \\
\hline Pendimethalin & $\mathrm{H}$ & 364,061 & 396,162 & 1.76 & 1.84 & 0.1 & 1.17 & 3.39 \\
\hline Metolachlor & $\mathrm{H}$ & 346,057 & 192,913 & 0.6 & 0.62 & 0.09 & 1.11 & 1.27 \\
\hline Ethion & I & 338,884 & 93,457 & 39.03 & 37.51 & 0.09 & 1.09 & 41.25 \\
\hline \multicolumn{9}{|c|}{ Great Salt Lake Basins (GRSL) } \\
\hline \multicolumn{9}{|c|}{ [Study Unit Area: 9,296,353 acres; Area in Cropland and Pasture: 758,301 acres] } \\
\hline Sulfur & $\mathrm{F}$ & 245,116 & 3,247 & 0.28 & 0.13 & 0.32 & 27.35 & 0.4 \\
\hline 2,4-D & $\mathrm{H}$ & 136,671 & 247,728 & 0.37 & 0.32 & 0.18 & 15.25 & 1.02 \\
\hline Sulfuric acid & $\mathrm{OP}$ & 61,962 & 217 & 0.24 & 0.21 & 0.08 & 6.91 & 0.29 \\
\hline Oil & I & 61,439 & 2,150 & 0.12 & 0.16 & 0.08 & 6.85 & 0.16 \\
\hline Metam sodium & $\mathrm{OP}$ & 31,476 & 203 & 0.1 & 0.05 & 0.04 & 3.51 & 0.13 \\
\hline Carbofuran & I & 25,764 & 39,951 & 0.5 & 0.61 & 0.03 & 2.87 & 1.14 \\
\hline Trifluralin & $\mathrm{H}$ & 25,529 & 13,430 & 0.13 & 0.05 & 0.03 & 2.85 & 0.26 \\
\hline Hexazinone & $\mathrm{H}$ & 22,799 & 22,236 & 4.94 & 3.82 & 0.03 & 2.54 & 14.3 \\
\hline $1,3-\mathrm{D}$ & OP & 20,403 & 123 & 0.05 & 0.03 & 0.03 & 2.28 & 0.08 \\
\hline MCPA & $\mathrm{H}$ & 17,996 & 39,171 & 0.39 & 0.32 & 0.02 & 2.01 & 0.8 \\
\hline Triallate & $\mathrm{H}$ & 16,992 & 15,147 & 0.74 & 0.68 & 0.02 & 1.9 & 1.7 \\
\hline Propargite & I & 13,652 & 6,966 & 0.4 & 0.36 & 0.02 & 1.52 & 0.63 \\
\hline Diclofop & $\mathrm{H}$ & 12,971 & 12,410 & 1.16 & 0.88 & 0.02 & 1.45 & 2.09 \\
\hline Metribuzin & $\mathrm{H}$ & 12,258 & 18,584 & 0.45 & 0.22 & 0.02 & 1.37 & 0.74 \\
\hline Alachlor & $\mathrm{H}$ & 11,981 & 3,977 & 0.05 & 0.03 & 0.02 & 1.34 & 0.11 \\
\hline Diuron & $\mathrm{H}$ & 10,383 & 8,643 & 0.28 & 0.27 & 0.01 & 1.16 & 0.38 \\
\hline Disulfoton & I & 9,453 & 12,958 & 0.53 & 0.55 & 0.01 & 1.05 & 1.11 \\
\hline Dicamba & $\mathrm{H}$ & 9,176 & 37,128 & 0.1 & 0.1 & 0.01 & 1.02 & 0.23 \\
\hline EPTC & $\mathrm{H}$ & 9,090 & 2,480 & 0.06 & 0.06 & 0.01 & 1.01 & 0.14 \\
\hline \multicolumn{9}{|c|}{ Hudson River Basin (HDSN) } \\
\hline \multicolumn{9}{|c|}{ [Study Unit Area: 8,552,050 acres; Area in Cropland and Pasture: 753,039 acres] } \\
\hline Oil & I & 464,851 & 16,264 & 0.87 & 1.2 & 0.62 & 23.65 & 1.2 \\
\hline Mancozeb & $\mathrm{F}$ & 164,360 & 21,995 & 1.98 & 1.08 & 0.22 & 8.36 & 3.06 \\
\hline Pendimethalin & $\mathrm{H}$ & 163,395 & 90,949 & 0.79 & 0.42 & 0.22 & 8.31 & 1.52 \\
\hline Atrazine & $\mathrm{H}$ & 116,847 & 75,416 & 0.18 & 0.13 & 0.16 & 5.95 & 0.42 \\
\hline Sulfur & $\mathrm{F}$ & 108,326 & 8,981 & 0.13 & 0.36 & 0.14 & 5.51 & 0.18 \\
\hline Captan & $\mathrm{F}$ & 96,773 & 15,287 & 2.55 & 2.61 & 0.13 & 4.92 & 4.97 \\
\hline Chlorpyrifos & I & 87,035 & 60,912 & 0.57 & 0.44 & 0.12 & 4.43 & 1.08 \\
\hline Alachlor & $\mathrm{H}$ & 85,476 & 51,889 & 0.33 & 0.36 & 0.11 & 4.35 & 0.75 \\
\hline Metolachlor & $\mathrm{H}$ & 84,941 & 41,507 & 0.15 & 0.13 & 0.11 & 4.32 & 0.31 \\
\hline Terbufos & I & 58,016 & 36,329 & 0.81 & 0.54 & 0.08 & 2.95 & 1.88 \\
\hline Cyanazine & $\mathrm{H}$ & 52,517 & 18,508 & 0.18 & 0.12 & 0.07 & 2.67 & 0.38 \\
\hline Glyphosate & $\mathrm{H}$ & 50,508 & 55,278 & 0.32 & 0.23 & 0.07 & 2.57 & 0.61 \\
\hline Chlorothalonil & $\mathrm{F}$ & 32,567 & 5,840 & 0.25 & 0.19 & 0.04 & 1.66 & 0.36 \\
\hline Dicamba & $\mathrm{H}$ & 28,038 & 38,056 & 0.29 & 0.1 & 0.04 & 1.43 & 0.69 \\
\hline Propargite & I & 25,093 & 10,594 & 0.73 & 0.55 & 0.03 & 1.28 & 1.16 \\
\hline Azinphos methyl & I & 24,506 & 14,040 & 0.98 & 0.54 & 0.03 & 1.25 & 1.75 \\
\hline
\end{tabular}


Table 5. Ranking of pesticides used in the National W ater-Quality Assessment Program study units during 1992-1995- Continued

\begin{tabular}{|c|c|c|c|c|c|c|c|c|}
\hline Compound & $\begin{array}{c}\text { Pesticide } \\
\text { type }\end{array}$ & $\begin{array}{l}\text { Average } \\
\text { amount } \\
\text { applied } \\
\text { (lb a.i.) }\end{array}$ & $\begin{array}{l}\text { Study unit } \\
\text { area treated } \\
\quad \text { (acres) }\end{array}$ & $\begin{array}{c}\text { Percentage of } \\
\text { national use }\end{array}$ & $\begin{array}{l}\text { Percentage of } \\
\text { U.S. area } \\
\text { treated }\end{array}$ & $\begin{array}{c}\text { Average } \\
\text { amount } \\
\text { applied to } \\
\text { cropland and } \\
\text { pasture area } \\
\text { (lb a.i.) }\end{array}$ & $\begin{array}{l}\text { Percentage of } \\
\text { all reported } \\
\text { pesticides } \\
\text { used in the } \\
\text { study unit }\end{array}$ & $\begin{array}{r}\text { Percenta } \\
\text { total } u \\
\text { among } s \\
\text { units }\end{array}$ \\
\hline \multicolumn{9}{|c|}{ Kanawha-New River Basin (KANA) } \\
\hline \multicolumn{9}{|c|}{ [Study Unit Area: 7,850,595 acres; Area in Cropland and Pasture: 724,871 acres] } \\
\hline $1,3-\mathrm{D}$ & $\mathrm{OP}$ & 82,228 & 1,175 & 0.21 & 0.24 & 0.11 & 16.55 & 0.34 \\
\hline 2,4-D & $\mathrm{H}$ & 72,432 & 114,116 & 0.2 & 0.15 & 0.1 & 14.58 & 0.54 \\
\hline Atrazine & $\mathrm{H}$ & 42,282 & 31,304 & 0.07 & 0.06 & 0.06 & 8.51 & 0.15 \\
\hline Oil & I & 32,388 & 991 & 0.06 & 0.07 & 0.04 & 6.52 & 0.08 \\
\hline Metolachlor & $\mathrm{H}$ & 31,420 & 16,884 & 0.05 & 0.05 & 0.04 & 6.32 & 0.12 \\
\hline Methyl bromide & $\mathrm{OP}$ & 30,419 & 75 & 0.08 & 0.04 & 0.04 & 6.12 & 0.11 \\
\hline Alachlor & $\mathrm{H}$ & 24,282 & 13,289 & 0.1 & 0.09 & 0.03 & 4.89 & 0.21 \\
\hline Sulfur & $\mathrm{F}$ & 19,500 & 564 & 0.02 & 0.02 & 0.03 & 3.93 & 0.03 \\
\hline Chlorpyrifos & I & 12,239 & 9,983 & 0.08 & 0.07 & 0.02 & 2.46 & 0.15 \\
\hline Simazine & $\mathrm{H}$ & 9,238 & 8,062 & 0.19 & 0.24 & 0.01 & 1.86 & 0.3 \\
\hline Captan & $\mathrm{F}$ & 8,750 & 940 & 0.23 & 0.16 & 0.01 & 1.76 & 0.45 \\
\hline Carbofuran & I & 8,558 & 10,564 & 0.17 & 0.16 & 0.01 & 1.72 & 0.38 \\
\hline Maleic hydrazide & $\mathrm{OP}$ & 7,229 & 2,340 & 0.34 & 0.32 & 0.01 & 1.46 & 0.67 \\
\hline Terbufos & I & 7,048 & 6,555 & 0.1 & 0.1 & 0.01 & 1.42 & 0.23 \\
\hline Carbaryl & I & 6,951 & 6,895 & 0.15 & 0.23 & 0.01 & 1.4 & 0.3 \\
\hline Acephate & I & 6,451 & 3,013 & 0.18 & 0.1 & 0.01 & 1.3 & 0.31 \\
\hline Ziram & $\mathrm{F}$ & 5,688 & 785 & 0.21 & 0.16 & 0.01 & 1.14 & 0.26 \\
\hline Mancozeb & $\mathrm{F}$ & 5,385 & 1,085 & 0.07 & 0.05 & 0.01 & 1.08 & 0.1 \\
\hline Dicamba & $\mathrm{H}$ & 5,156 & 16,385 & 0.05 & 0.04 & 0.01 & 1.04 & 0.13 \\
\hline Methomyl & I & 4,996 & 8,508 & 0.2 & 0.25 & 0.01 & 1.01 & 0.34 \\
\hline \multicolumn{9}{|c|}{ Kentucky River Basin (KNTY) } \\
\hline \multicolumn{9}{|c|}{ [Study Unit Area: 4,457,860 acres; Area in Cropland and Pasture: $1,142,120$ acres] } \\
\hline Methyl bromide & $\mathrm{OP}$ & 340,807 & 684 & 0.87 & 0.34 & 0.3 & 27.73 & 1.21 \\
\hline Maleic hydrazide & $\mathrm{OP}$ & 162,456 & 52,405 & 7.64 & 7.26 & 0.14 & 13.22 & 15.03 \\
\hline Acephate & I & 152,516 & 68,085 & 4.19 & 2.14 & 0.13 & 12.41 & 7.39 \\
\hline Atrazine & $\mathrm{H}$ & 70,910 & 46,722 & 0.11 & 0.08 & 0.06 & 5.77 & 0.25 \\
\hline $2,4-\mathrm{D}$ & $\mathrm{H}$ & 51,163 & 75,874 & 0.14 & 0.1 & 0.04 & 4.16 & 0.38 \\
\hline Metolachlor & $\mathrm{H}$ & 45,379 & 29,623 & 0.08 & 0.1 & 0.04 & 3.69 & 0.17 \\
\hline Butylate & $\mathrm{H}$ & 40,406 & 9,485 & 0.53 & 0.48 & 0.04 & 3.29 & 1.36 \\
\hline Pebulate & $\mathrm{H}$ & 38,113 & 9,528 & 6.8 & 6.06 & 0.03 & 3.1 & 11.81 \\
\hline Carbofuran & I & 36,235 & 22,076 & 0.71 & 0.34 & 0.03 & 2.95 & 1.6 \\
\hline Chloropicrin & OP & 34,196 & 684 & 0.46 & 0.56 & 0.03 & 2.78 & 0.8 \\
\hline Metalaxyl & $\mathrm{F}$ & 34,062 & 68,086 & 3.84 & 2.78 & 0.03 & 2.77 & 6.54 \\
\hline Pendimethalin & $\mathrm{H}$ & 27,095 & 36,145 & 0.13 & 0.17 & 0.02 & 2.2 & 0.25 \\
\hline Chlorpyrifos & I & 21,650 & 13,407 & 0.14 & 0.1 & 0.02 & 1.76 & 0.27 \\
\hline Fenamiphos & I & 21,098 & 21,098 & 3.18 & 4.38 & 0.02 & 1.72 & 6.43 \\
\hline Simazine & $\mathrm{H}$ & 19,726 & 17,567 & 0.41 & 0.51 & 0.02 & 1.61 & 0.64 \\
\hline Alachlor & $\mathrm{H}$ & 17,346 & 8,701 & 0.07 & 0.06 & 0.02 & 1.41 & 0.15 \\
\hline Napropamide & $\mathrm{H}$ & 14,995 & 14,980 & 3.16 & 4.19 & 0.01 & 1.22 & 5.03 \\
\hline Isopropalin & $\mathrm{H}$ & 12,931 & 12,931 & 9.28 & 9.29 & 0.01 & 1.05 & 19.52 \\
\hline
\end{tabular}


Table 5. Ranking of pesticides used in the National W ater-Quality Assessment Program study units during 1992-1995- Continued

\begin{tabular}{|c|c|c|c|c|c|c|c|c|}
\hline Compound & $\begin{array}{c}\text { Pesticide } \\
\text { type }\end{array}$ & $\begin{array}{l}\text { Average } \\
\text { amount } \\
\text { applied } \\
\text { (lb a.i.) }\end{array}$ & $\begin{array}{l}\text { Study unit } \\
\text { area treated } \\
\text { (acres) }\end{array}$ & $\begin{array}{l}\text { Percentage of } \\
\text { national use }\end{array}$ & $\begin{array}{l}\text { Percentage of } \\
\text { U.S. area } \\
\text { treated }\end{array}$ & $\begin{array}{c}\text { Average } \\
\text { amount } \\
\text { applied to } \\
\text { cropland and } \\
\text { pasture area } \\
\text { (lb a.i.) }\end{array}$ & $\begin{array}{l}\text { Percentage of } \\
\text { all reported } \\
\text { pesticides } \\
\text { used in the } \\
\text { study unit }\end{array}$ & $\begin{array}{c}\text { Percentage } \\
\text { total use } \\
\text { among stuc } \\
\text { units }\end{array}$ \\
\hline \multicolumn{9}{|c|}{ Lake Erie-Lake Saint Clair Drainage (LERI) } \\
\hline \multicolumn{9}{|c|}{ [Study Unit Area: 13,333,230 acres; Area in Cropland and Pasture: 5,883,633 acres] } \\
\hline Metolachlor & $\mathrm{H}$ & $2,216,737$ & $1,145,825$ & 3.83 & 3.67 & 0.38 & 17.01 & 8.14 \\
\hline Atrazine & $\mathrm{H}$ & $1,851,634$ & $1,539,853$ & 2.9 & 2.7 & 0.31 & 14.21 & 6.59 \\
\hline Alachlor & $\mathrm{H}$ & $1,237,672$ & 647,554 & 4.83 & 4.46 & 0.21 & 9.5 & 10.84 \\
\hline Cyanazine & $\mathrm{H}$ & 880,058 & 435,381 & 2.98 & 2.75 & 0.15 & 6.75 & 6.35 \\
\hline Acetochlor & $\mathrm{H}$ & 739,783 & 374,197 & 3.1 & 3.16 & 0.13 & 5.68 & 6.68 \\
\hline Pendimethalin & $\mathrm{H}$ & 598,269 & 615,039 & 2.89 & 2.86 & 0.1 & 4.59 & 5.58 \\
\hline Glyphosate & $\mathrm{H}$ & 561,964 & 904,199 & 3.5 & 3.8 & 0.1 & 4.31 & 6.76 \\
\hline $2,4-\mathrm{D}$ & $\mathrm{H}$ & 307,424 & 673,121 & 0.83 & 0.87 & 0.05 & 2.36 & 2.29 \\
\hline Mancozeb & $\mathrm{F}$ & 277,705 & 48,063 & 3.35 & 2.35 & 0.05 & 2.13 & 5.18 \\
\hline Bentazon & $\mathrm{H}$ & 258,364 & 334,993 & 3.84 & 3.34 & 0.04 & 1.98 & 6.65 \\
\hline Butylate & $\mathrm{H}$ & 257,012 & 74,819 & 3.34 & 3.77 & 0.04 & 1.97 & 8.64 \\
\hline Linuron & $\mathrm{H}$ & 253,303 & 378,807 & 12.83 & 12.03 & 0.04 & 1.94 & 23.4 \\
\hline Chlorothalonil & $\mathrm{F}$ & 207,283 & 30,014 & 1.62 & 0.96 & 0.04 & 1.59 & 2.3 \\
\hline Dicamba & $\mathrm{H}$ & 206,425 & 645,043 & 2.15 & 1.66 & 0.04 & 1.58 & 5.08 \\
\hline EPTC & $\mathrm{H}$ & 204,155 & 58,140 & 1.45 & 1.45 & 0.03 & 1.57 & 3.1 \\
\hline Chlorpyrifos & I & 202,600 & 186,745 & 1.32 & 1.34 & 0.03 & 1.55 & 2.52 \\
\hline Metribuzin & $\mathrm{H}$ & 182,152 & 489,213 & 6.74 & 5.82 & 0.03 & 1.4 & 11.02 \\
\hline Trifluralin & $\mathrm{H}$ & 168,679 & 180,511 & 0.85 & 0.71 & 0.03 & 1.29 & 1.72 \\
\hline Maneb & $\mathrm{F}$ & 160,829 & 26,800 & 5.35 & 3.81 & 0.03 & 1.23 & 9.36 \\
\hline Oil & I & 155,216 & 4,426 & 0.29 & 0.33 & 0.03 & 1.19 & 0.4 \\
\hline Terbufos & I & 144,680 & 128,341 & 2.01 & 1.92 & 0.02 & 1.11 & 4.69 \\
\hline \multicolumn{9}{|c|}{ Long Island-New Jersey Coastal Drainages (LINJ) } \\
\hline \multicolumn{9}{|c|}{ [Study Unit Area: 3,811,281 acres; Area in Cropland and Pasture; 239,471 acres] } \\
\hline Sulfur & $\mathrm{F}$ & 266,031 & 4,872 & 0.31 & 0.2 & 1.11 & 25.27 & 0.44 \\
\hline Metolachlor & $\mathrm{H}$ & 95,993 & 50,505 & 0.17 & 0.16 & 0.4 & 9.12 & 0.35 \\
\hline Oil & I & 70,187 & 2,746 & 0.13 & 0.2 & 0.29 & 6.67 & 0.18 \\
\hline Mancozeb & $\mathrm{F}$ & 57,603 & 11,130 & 0.69 & 0.55 & 0.24 & 5.47 & 1.07 \\
\hline Captan & $\mathrm{F}$ & 50,325 & 9,731 & 1.32 & 1.66 & 0.21 & 4.78 & 2.58 \\
\hline Atrazine & $\mathrm{H}$ & 47,853 & 33,358 & 0.08 & 0.06 & 0.2 & 4.55 & 0.17 \\
\hline Chlorothalonil & $\mathrm{F}$ & 44,319 & 10,446 & 0.35 & 0.33 & 0.19 & 4.21 & 0.49 \\
\hline Maneb & $\mathrm{F}$ & 27,643 & 5,494 & 0.92 & 0.78 & 0.12 & 2.63 & 1.61 \\
\hline Alachlor & $\mathrm{H}$ & 23,128 & 12,199 & 0.09 & 0.08 & 0.1 & 2.2 & 0.2 \\
\hline $2,4-\mathrm{D}$ & $\mathrm{H}$ & 22,887 & 41,951 & 0.06 & 0.05 & 0.1 & 2.17 & 0.17 \\
\hline Glyphosate & $\mathrm{H}$ & 17,741 & 14,275 & 0.11 & 0.06 & 0.07 & 1.69 & 0.21 \\
\hline Methomyl & $\mathrm{I}$ & 16,875 & 9,812 & 0.69 & 0.29 & 0.07 & 1.6 & 1.16 \\
\hline Methyl bromide & OP & 13,369 & 84 & 0.03 & 0.04 & 0.06 & 1.27 & 0.05 \\
\hline Bensulide & $\mathrm{H}$ & 13,210 & 3,072 & 4.1 & 3.33 & 0.06 & 1.25 & 9.48 \\
\hline Cryolite & I & 12,995 & 975 & 0.42 & 0.2 & 0.05 & 1.23 & 0.61 \\
\hline Linuron & $\mathrm{H}$ & 12,747 & 23,901 & 0.65 & 0.76 & 0.05 & 1.21 & 1.18 \\
\hline Metiram & $\mathrm{F}$ & 12,292 & 1,308 & 1.33 & 1.03 & 0.05 & 1.17 & 2.92 \\
\hline
\end{tabular}


Table 5. Ranking of pesticides used in the National W ater-Quality Assessment Program study units during 1992-1995- Continued

\begin{tabular}{|c|c|c|c|c|c|c|c|c|}
\hline Compound & $\begin{array}{c}\text { Pesticide } \\
\text { type }\end{array}$ & $\begin{array}{l}\text { Average } \\
\text { amount } \\
\text { applied } \\
\text { (lb a.i.) }\end{array}$ & $\begin{array}{l}\text { Study unit } \\
\text { area treated } \\
\text { (acres) }\end{array}$ & $\begin{array}{c}\text { Percentage of } \\
\text { national use }\end{array}$ & $\begin{array}{l}\text { Percentage of } \\
\text { U.S. area } \\
\text { treated }\end{array}$ & $\begin{array}{c}\text { Average } \\
\text { amount } \\
\text { applied to } \\
\text { cropland and } \\
\text { pasture area } \\
\text { (lb a.i.) }\end{array}$ & $\begin{array}{c}\text { Percentage of } \\
\text { all reported } \\
\text { pesticides } \\
\text { used in the } \\
\text { study unit }\end{array}$ & $\begin{array}{c}\text { Percentage of } \\
\text { total use } \\
\text { among study } \\
\text { units }\end{array}$ \\
\hline \multicolumn{9}{|c|}{ Long Island-New Jersey Coastal Drainages (LINJ)—Continued } \\
\hline Diuron & $\mathrm{H}$ & 11,030 & 6,000 & 0.29 & 0.19 & 0.05 & 1.05 & 0.41 \\
\hline Chlorpyrifos & I & 10,880 & 7,256 & 0.07 & 0.05 & 0.05 & 1.03 & 0.14 \\
\hline Carbaryl & I & 10,712 & 4,195 & 0.24 & 0.14 & 0.04 & 1.02 & 0.46 \\
\hline \multicolumn{9}{|c|}{ Lower Illinois River Basin (LIRB) } \\
\hline \multicolumn{9}{|c|}{ [Study Unit Area: 11,478,316 acres; Area in Cropland and Pasture: 8,025,297 acres] } \\
\hline Atrazine & $\mathrm{H}$ & $3,097,037$ & $2,814,321$ & 4.84 & 4.94 & 0.39 & 16.86 & 11.03 \\
\hline Metolachlor & $\mathrm{H}$ & $2,944,265$ & $1,421,943$ & 5.08 & 4.55 & 0.37 & 16.03 & 10.82 \\
\hline Cyanazine & $\mathrm{H}$ & $2,367,499$ & 921,324 & 8.02 & 5.81 & 0.3 & 12.89 & 17.09 \\
\hline Acetochlor & $\mathrm{H}$ & $1,938,830$ & 959,817 & 8.13 & 8.1 & 0.24 & 10.56 & 17.5 \\
\hline Pendimethalin & $\mathrm{H}$ & $1,527,056$ & $1,539,983$ & 7.37 & 7.16 & 0.19 & 8.32 & 14.23 \\
\hline Alachlor & $\mathrm{H}$ & 997,006 & 450,655 & 3.89 & 3.1 & 0.12 & 5.43 & 8.73 \\
\hline EPTC & $\mathrm{H}$ & 521,351 & 120,163 & 3.7 & 2.99 & 0.06 & 2.84 & 7.91 \\
\hline Glyphosate & $\mathrm{H}$ & 508,989 & 912,395 & 3.17 & 3.83 & 0.06 & 2.77 & 6.12 \\
\hline $2,4-\mathrm{D}$ & $\mathrm{H}$ & 477,967 & $1,199,934$ & 1.28 & 1.54 & 0.06 & 2.6 & 3.56 \\
\hline Trifluralin & $\mathrm{H}$ & 420,950 & 548,113 & 2.13 & 2.16 & 0.05 & 2.29 & 4.29 \\
\hline Simazine & $\mathrm{H}$ & 375,425 & 280,540 & 7.81 & 8.18 & 0.05 & 2.04 & 12.23 \\
\hline Dicamba & $\mathrm{H}$ & 358,065 & 918,272 & 3.73 & 2.36 & 0.04 & 1.95 & 8.8 \\
\hline Chlorpyrifos & I & 339,987 & 336,253 & 2.21 & 2.41 & 0.04 & 1.85 & 4.22 \\
\hline Terbufos & I & 334,613 & 281,194 & 4.65 & 4.21 & 0.04 & 1.82 & 10.85 \\
\hline Bentazon & $\mathrm{H}$ & 291,302 & 503,289 & 4.33 & 5.02 & 0.04 & 1.59 & 7.5 \\
\hline Butylate & $\mathrm{H}$ & 259,156 & 79,986 & 3.37 & 4.03 & 0.03 & 1.41 & 8.71 \\
\hline \multicolumn{9}{|c|}{ Lower Susquehanna River Basin (LSUS) } \\
\hline \multicolumn{9}{|c|}{ [Study Unit Area: 5,969,969 acres; Area in Cropland and Pasture: $1,544,414$ acres] } \\
\hline Metolachlor & $\mathrm{H}$ & 758,964 & 425,738 & 1.31 & 1.36 & 0.49 & 21.69 & 2.79 \\
\hline Atrazine & $\mathrm{H}$ & 518,195 & 464,045 & 0.81 & 0.81 & 0.34 & 14.81 & 1.85 \\
\hline Oil & I & 357,076 & 11,556 & 0.67 & 0.85 & 0.23 & 10.2 & 0.92 \\
\hline Pendimethalin & $\mathrm{H}$ & 209,512 & 186,043 & 1.01 & 0.87 & 0.14 & 5.99 & 1.95 \\
\hline Alachlor & $\mathrm{H}$ & 170,231 & 95,619 & 0.66 & 0.66 & 0.11 & 4.86 & 1.49 \\
\hline Captan & $\mathrm{F}$ & 129,002 & 17,906 & 3.39 & 3.06 & 0.08 & 3.69 & 6.62 \\
\hline Sulfur & $\mathrm{F}$ & 121,387 & 5,906 & 0.14 & 0.24 & 0.08 & 3.47 & 0.2 \\
\hline Cyanazine & $\mathrm{H}$ & 119,132 & 77,896 & 0.4 & 0.49 & 0.08 & 3.4 & 0.86 \\
\hline Chlorpyrifos & I & 102,273 & 95,068 & 0.66 & 0.68 & 0.07 & 2.92 & 1.27 \\
\hline Methyl bromide & OP & 82,828 & 250 & 0.21 & 0.12 & 0.05 & 2.37 & 0.3 \\
\hline Glyphosate & $\mathrm{H}$ & 78,586 & 77,235 & 0.49 & 0.32 & 0.05 & 2.25 & 0.95 \\
\hline Simazine & $\mathrm{H}$ & 78,524 & 82,925 & 1.63 & 2.42 & 0.05 & 2.24 & 2.56 \\
\hline Mancozeb & $\mathrm{F}$ & 65,289 & 11,569 & 0.79 & 0.57 & 0.04 & 1.87 & 1.22 \\
\hline Dimethoate & I & 58,940 & 93,161 & 1.73 & 1.81 & 0.04 & 1.68 & 5.34 \\
\hline $2,4-\mathrm{D}$ & $\mathrm{H}$ & 53,912 & 114,700 & 0.15 & 0.15 & 0.03 & 1.54 & 0.4 \\
\hline Carbofuran & I & 42,636 & 50,156 & 0.83 & 0.77 & 0.03 & 1.22 & 1.88 \\
\hline Ziram & $\mathrm{F}$ & 40,316 & 4,943 & 1.46 & 1 & 0.03 & 1.15 & 1.87 \\
\hline
\end{tabular}


Table 5. Ranking of pesticides used in the National W ater-Quality Assessment Program study units during 1992-1995- Continued

\begin{tabular}{|c|c|c|c|c|c|c|c|c|}
\hline Compound & $\begin{array}{l}\text { Pesticide } \\
\text { type }\end{array}$ & $\begin{array}{l}\text { Average } \\
\text { amount } \\
\text { applied } \\
\text { (lb a.i.) }\end{array}$ & $\begin{array}{l}\text { Study unit } \\
\text { area treated } \\
\quad \text { (acres) }\end{array}$ & $\begin{array}{l}\text { Percentage of } \\
\text { national use }\end{array}$ & $\begin{array}{c}\text { Percentage of } \\
\text { U.S. area } \\
\text { treated }\end{array}$ & $\begin{array}{c}\text { Average } \\
\text { amount } \\
\text { applied to } \\
\text { cropland and } \\
\text { pasture area } \\
\text { (lb a.i.) }\end{array}$ & $\begin{array}{l}\text { Percentage of } \\
\text { all reported } \\
\text { pesticides } \\
\text { used in the } \\
\text { study unit }\end{array}$ & $\begin{array}{r}\text { Percents } \\
\text { total t } \\
\text { among s } \\
\text { unit }\end{array}$ \\
\hline \multicolumn{9}{|c|}{ Lower Tennessee River Basin (LTEN) } \\
\hline \multicolumn{9}{|c|}{ [Study Unit Area: 12,490,377 acres; Area in Cropland and Pasture: 2,661,003 acres] } \\
\hline Atrazine & $\mathrm{H}$ & 460,274 & 306,980 & 0.72 & 0.54 & 0.17 & 11.3 & 1.64 \\
\hline MSMA & $\mathrm{H}$ & 311,882 & 161,860 & 5.41 & 4.25 & 0.12 & 7.66 & 6.66 \\
\hline 2,4-D & $\mathrm{H}$ & 224,732 & 393,639 & 0.6 & 0.51 & 0.08 & 5.52 & 1.67 \\
\hline Metolachlor & $\mathrm{H}$ & 201,293 & 132,197 & 0.35 & 0.42 & 0.08 & 4.94 & 0.74 \\
\hline Methyl parathion & I & 174,152 & 115,111 & 1.95 & 1.54 & 0.07 & 4.28 & 3.14 \\
\hline Fluometuron & $\mathrm{H}$ & 143,411 & 179,062 & 3.19 & 3.59 & 0.05 & 3.52 & 4.13 \\
\hline Trifluralin & $\mathrm{H}$ & 140,088 & 211,127 & 0.71 & 0.83 & 0.05 & 3.44 & 1.43 \\
\hline Alachlor & $\mathrm{H}$ & 139,493 & 81,662 & 0.54 & 0.56 & 0.05 & 3.43 & 1.22 \\
\hline Butylate & $\mathrm{H}$ & 132,976 & 34,984 & 1.73 & 1.76 & 0.05 & 3.27 & 4.47 \\
\hline Methyl bromide & OP & 117,903 & 411 & 0.3 & 0.2 & 0.04 & 2.9 & 0.42 \\
\hline Pendimethalin & $\mathrm{H}$ & 113,985 & 146,676 & 0.55 & 0.68 & 0.04 & 2.8 & 1.06 \\
\hline Cyanazine & $\mathrm{H}$ & 113,409 & 124,814 & 0.38 & 0.79 & 0.04 & 2.78 & 0.82 \\
\hline Glyphosate & $\mathrm{H}$ & 105,289 & 161,844 & 0.66 & 0.68 & 0.04 & 2.59 & 1.27 \\
\hline Thiodicarb & I & 98,127 & 142,500 & 6.59 & 7.26 & 0.04 & 2.41 & 9.14 \\
\hline Tribufos & OP & 97,098 & 104,621 & 2.61 & 2.63 & 0.04 & 2.38 & 3.66 \\
\hline $1,3-\mathrm{D}$ & OP & 83,342 & 2,193 & 0.21 & 0.45 & 0.03 & 2.05 & 0.34 \\
\hline Norflurazon & $\mathrm{H}$ & 83,149 & 111,734 & 3.66 & 4.66 & 0.03 & 2.04 & 4.42 \\
\hline Ethephon & OP & 79,809 & 53,739 & 2.59 & 1.83 & 0.03 & 1.96 & 3.4 \\
\hline Aldicarb & I & 78,975 & 152,306 & 1.84 & 3.53 & 0.03 & 1.94 & 2.48 \\
\hline Sodium chlorate & OP & 70,308 & 20,096 & 1.34 & 1.17 & 0.03 & 1.73 & 1.74 \\
\hline Simazine & $\mathrm{H}$ & 65,422 & 52,727 & 1.36 & 1.54 & 0.02 & 1.61 & 2.13 \\
\hline PCNB & $\mathrm{F}$ & 64,793 & 80,910 & 3.82 & 5.1 & 0.02 & 1.59 & 5.29 \\
\hline Malathion & I & 63,075 & 52,968 & 2.03 & 2.74 & 0.02 & 1.55 & 4.38 \\
\hline DSMA & $\mathrm{H}$ & 61,620 & 21,101 & 4.42 & 2.75 & 0.02 & 1.51 & 5.57 \\
\hline Profenofos & I & 61,352 & 61,334 & 2.89 & 3.62 & 0.02 & 1.51 & 4.2 \\
\hline Acephate & I & 60,577 & 94,084 & 1.67 & 2.95 & 0.02 & 1.49 & 2.93 \\
\hline Chlorpyrifos & I & 47,062 & 53,838 & 0.31 & 0.39 & 0.02 & 1.16 & 0.59 \\
\hline Sulfur & $\mathrm{F}$ & 41,003 & 734 & 0.05 & 0.03 & 0.02 & 1.01 & 0.07 \\
\hline
\end{tabular}

Great and Little Miami River Basins (MIAM)

[Study Unit Area: 4,699,665 acres; Area in Cropland and Pasture: 2,424,660 acres]

\begin{tabular}{|c|c|c|c|c|c|c|c|c|}
\hline Atrazine & $\mathrm{H}$ & $1,042,409$ & 843,791 & 1.63 & 1.48 & 0.43 & 19.4 & 3.71 \\
\hline Metolachlor & $\mathrm{H}$ & 966,176 & 498,051 & 1.67 & 1.59 & 0.4 & 17.98 & 3.55 \\
\hline Cyanazine & $\mathrm{H}$ & 471,913 & 220,984 & 1.6 & 1.4 & 0.19 & 8.78 & 3.41 \\
\hline Acetochlor & $\mathrm{H}$ & 439,270 & 216,950 & 1.84 & 1.83 & 0.18 & 8.18 & 3.96 \\
\hline Alachlor & $\mathrm{H}$ & 439,130 & 227,553 & 1.71 & 1.57 & 0.18 & 8.17 & 3.85 \\
\hline Glyphosate & $\mathrm{H}$ & 250,324 & 426,408 & 1.56 & 1.79 & 0.1 & 4.66 & 3.01 \\
\hline Pendimethalin & $\mathrm{H}$ & 238,539 & 255,835 & 1.15 & 1.19 & 0.1 & 4.44 & 2.22 \\
\hline Butylate & $\mathrm{H}$ & 182,492 & 51,781 & 2.37 & 2.61 & 0.08 & 3.4 & 6.13 \\
\hline $2,4-\mathrm{D}$ & $\mathrm{H}$ & 145,671 & 306,503 & 0.39 & 0.39 & 0.06 & 2.71 & 1.09 \\
\hline Dicamba & $\mathrm{H}$ & 104,695 & 336,154 & 1.09 & 0.86 & 0.04 & 1.95 & 2.57 \\
\hline Bentazon & $\mathrm{H}$ & 94,904 & 124,668 & 1.41 & 1.24 & 0.04 & 1.77 & 2.44 \\
\hline
\end{tabular}


Table 5. Ranking of pesticides used in the National W ater-Quality Assessment Program study units during 1992-1995- Continued

\begin{tabular}{|c|c|c|c|c|c|c|c|c|}
\hline Compound & $\begin{array}{c}\text { Pesticide } \\
\text { type }\end{array}$ & $\begin{array}{l}\text { Average } \\
\text { amount } \\
\text { applied } \\
\text { (lb a.i.) }\end{array}$ & $\begin{array}{l}\text { Study unit } \\
\text { area treated } \\
\text { (acres) }\end{array}$ & $\begin{array}{l}\text { Percentage of } \\
\text { national use }\end{array}$ & $\begin{array}{c}\text { Percentage of } \\
\text { U.S. area } \\
\text { treated }\end{array}$ & $\begin{array}{c}\text { Average } \\
\text { amount } \\
\text { applied to } \\
\text { cropland and } \\
\text { pasture area } \\
\text { (lb a.i.) }\end{array}$ & $\begin{array}{l}\text { Percentage of } \\
\text { all reported } \\
\text { pesticides } \\
\text { used in the } \\
\text { study unit }\end{array}$ & $\begin{array}{c}\text { Percentage of } \\
\text { total use } \\
\text { among study } \\
\text { units }\end{array}$ \\
\hline \multicolumn{9}{|c|}{ Great and Little Miami River Basins (MIAM)—Continued } \\
\hline EPTC & $\mathrm{H}$ & 89,614 & 22,218 & 0.64 & 0.55 & 0.04 & 1.67 & 1.36 \\
\hline Chlorpyrifos & I & 84,644 & 79,940 & 0.55 & 0.57 & 0.03 & 1.58 & 1.05 \\
\hline Terbufos & I & 69,070 & 59,939 & 0.96 & 0.9 & 0.03 & 1.29 & 2.24 \\
\hline Simazine & $\mathrm{H}$ & 68,409 & 63,337 & 1.42 & 1.85 & 0.03 & 1.27 & 2.23 \\
\hline Trifluralin & $\mathrm{H}$ & 60,294 & 58,147 & 0.31 & 0.23 & 0.02 & 1.12 & 0.62 \\
\hline Metribuzin & $\mathrm{H}$ & 55,746 & 173,436 & 2.06 & 2.06 & 0.02 & 1.04 & 3.37 \\
\hline Dimethenamid & $\mathrm{H}$ & 53,960 & 54,505 & 2.06 & 2.35 & 0.02 & 1 & 4.12 \\
\hline \multicolumn{9}{|c|}{ Mississippi Embayment (MISE) } \\
\hline \multicolumn{9}{|c|}{ [Study Unit Area: 31,778,108 acres; Area in Cropland and Pasture: $14,381,259$ acres] } \\
\hline Propanil & $\mathrm{H}$ & $4,227,967$ & $1,410,104$ & 58.48 & 60.31 & 0.29 & 8.89 & 72.75 \\
\hline Methyl parathion & I & $3,669,139$ & $1,882,710$ & 41.09 & 25.13 & 0.26 & 7.71 & 66.21 \\
\hline MSMA & $\mathrm{H}$ & $2,968,317$ & $2,091,627$ & 51.5 & 54.94 & 0.21 & 6.24 & 63.39 \\
\hline Trifluralin & $\mathrm{H}$ & $2,657,933$ & $3,556,741$ & 13.43 & 14.02 & 0.18 & 5.59 & 27.09 \\
\hline Fluometuron & $\mathrm{H}$ & $2,629,665$ & $2,930,077$ & 58.43 & 58.8 & 0.18 & 5.53 & 75.66 \\
\hline Metolachlor & $\mathrm{H}$ & $2,586,603$ & $1,610,940$ & 4.47 & 5.15 & 0.18 & 5.44 & 9.5 \\
\hline Atrazine & $\mathrm{H}$ & $1,867,881$ & $1,385,861$ & 2.92 & 2.43 & 0.13 & 3.93 & 6.65 \\
\hline Cyanazine & $\mathrm{H}$ & $1,599,680$ & $1,632,502$ & 5.42 & 10.3 & 0.11 & 3.36 & 11.55 \\
\hline Pendimethalin & $\mathrm{H}$ & $1,560,016$ & $2,022,508$ & 7.53 & 9.41 & 0.11 & 3.28 & 14.54 \\
\hline Molinate & $\mathrm{H}$ & $1,521,071$ & 469,316 & 31.02 & 33.05 & 0.11 & 3.2 & 37.44 \\
\hline Alachlor & $\mathrm{H}$ & $1,254,442$ & 792,668 & 4.89 & 5.46 & 0.09 & 2.64 & 10.98 \\
\hline Tribufos & $\mathrm{OP}$ & $1,094,015$ & $1,374,094$ & 29.4 & 34.54 & 0.08 & 2.3 & 41.23 \\
\hline Glyphosate & $\mathrm{H}$ & $1,091,804$ & $1,664,522$ & 6.81 & 6.99 & 0.08 & 2.3 & 13.13 \\
\hline Profenofos & I & 992,243 & 800,013 & 46.77 & 47.18 & 0.07 & 2.09 & 67.84 \\
\hline Ethephon & $\mathrm{OP}$ & 893,406 & 950,262 & 29.02 & 32.28 & 0.06 & 1.88 & 38.04 \\
\hline Norflurazon & $\mathrm{H}$ & 885,458 & $1,094,332$ & 38.98 & 45.67 & 0.06 & 1.86 & 47.07 \\
\hline $2,4-\mathrm{D}$ & $\mathrm{H}$ & 846,269 & $1,428,906$ & 2.27 & 1.84 & 0.06 & 1.78 & 6.3 \\
\hline Sodium chlorate & $\mathrm{OP}$ & 820,478 & 347,883 & 15.65 & 20.32 & 0.06 & 1.72 & 20.26 \\
\hline DSMA & $\mathrm{H}$ & 807,976 & 455,263 & 57.91 & 59.34 & 0.06 & 1.7 & 73.05 \\
\hline Aldicarb & I & 697,546 & $1,111,171$ & 16.29 & 25.75 & 0.05 & 1.47 & 21.94 \\
\hline Bentazon & $\mathrm{H}$ & 609,633 & $1,087,746$ & 9.06 & 10.86 & 0.04 & 1.28 & 15.7 \\
\hline PCNB & $\mathrm{F}$ & 599,920 & 723,819 & 35.39 & 45.63 & 0.04 & 1.26 & 48.96 \\
\hline Prometryn & $\mathrm{H}$ & 593,900 & $1,039,793$ & 32.18 & 40.63 & 0.04 & 1.25 & 57.6 \\
\hline Clomazone & $\mathrm{H}$ & 565,348 & $1,008,095$ & 28.82 & 28.96 & 0.04 & 1.19 & 48.26 \\
\hline Acifluorfen & $\mathrm{H}$ & 557,887 & $2,031,593$ & 32.98 & 27.98 & 0.04 & 1.17 & 48.64 \\
\hline Thiodicarb & I & 518,022 & 655,769 & 34.81 & 33.39 & 0.04 & 1.09 & 48.27 \\
\hline Thiobencarb & $\mathrm{H}$ & 484,686 & 171,502 & 33.74 & 36.24 & 0.03 & 1.02 & 52.1 \\
\hline \multicolumn{9}{|c|}{ Mobile River and Tributaries (MOBL) } \\
\hline \multicolumn{9}{|c|}{ [Study Unit Area: 27,970,254 acres; Area in Cropland and Pasture: 2,726,827 acres] } \\
\hline Methyl bromide & $\mathrm{OP}$ & 846,290 & 3,522 & 2.16 & 1.72 & 0.31 & 15.03 & 3.02 \\
\hline 2,4-D & $\mathrm{H}$ & 522,846 & 934,544 & 1.4 & 1.2 & 0.19 & 9.29 & 3.89 \\
\hline Sulfur & $\mathrm{F}$ & 381,553 & 10,166 & 0.44 & 0.41 & 0.14 & 6.78 & 0.63 \\
\hline MSMA & $\mathrm{H}$ & 296,019 & 161,291 & 5.14 & 4.24 & 0.11 & 5.26 & 6.32 \\
\hline
\end{tabular}


Table 5. Ranking of pesticides used in the National W ater-Quality Assessment Program study units during 1992-1995- Continued

\begin{tabular}{|c|c|c|c|c|c|c|c|c|}
\hline Compound & $\begin{array}{c}\text { Pesticide } \\
\text { type }\end{array}$ & $\begin{array}{l}\text { Average } \\
\text { amount } \\
\text { applied } \\
\text { (lb a.i.) }\end{array}$ & $\begin{array}{l}\text { Study unit } \\
\text { area treated } \\
\quad \text { (acres) }\end{array}$ & $\begin{array}{l}\text { Percentage of } \\
\text { national use }\end{array}$ & $\begin{array}{c}\text { Percentage of } \\
\text { U.S. area } \\
\text { treated }\end{array}$ & $\begin{array}{c}\text { Average } \\
\text { amount } \\
\text { applied to } \\
\text { cropland and } \\
\text { pasture area } \\
\text { (lb a.i.) }\end{array}$ & $\begin{array}{l}\text { Percentage of } \\
\text { all reported } \\
\text { pesticides } \\
\text { used in the } \\
\text { study unit }\end{array}$ & $\begin{array}{c}\text { Percentage of } \\
\text { total use } \\
\text { among study } \\
\text { units }\end{array}$ \\
\hline \multicolumn{9}{|c|}{ Mobile River and Tributaries (MOBL)—Continued } \\
\hline Methyl parathion & I & 245,118 & 159,802 & 2.75 & 2.13 & 0.09 & 4.35 & 4.42 \\
\hline Atrazine & $\mathrm{H}$ & 221,092 & 138,042 & 0.35 & 0.24 & 0.08 & 3.93 & 0.79 \\
\hline Trifluralin & $\mathrm{H}$ & 190,838 & 272,158 & 0.96 & 1.07 & 0.07 & 3.39 & 1.95 \\
\hline Pendimethalin & $\mathrm{H}$ & 169,324 & 206,871 & 0.82 & 0.96 & 0.06 & 3.01 & 1.58 \\
\hline Fluometuron & $\mathrm{H}$ & 152,422 & 181,739 & 3.39 & 3.65 & 0.06 & 2.71 & 4.39 \\
\hline Chloropicrin & OP & 139,215 & 2,175 & 1.87 & 1.77 & 0.05 & 2.47 & 3.24 \\
\hline Tribufos & OP & 106,723 & 120,107 & 2.87 & 3.02 & 0.04 & 1.9 & 4.02 \\
\hline Alachlor & $\mathrm{H}$ & 104,190 & 94,826 & 0.41 & 0.65 & 0.04 & 1.85 & 0.91 \\
\hline $1,3-\mathrm{D}$ & OP & 101,245 & 2,611 & 0.25 & 0.53 & 0.04 & 1.8 & 0.41 \\
\hline Glyphosate & $\mathrm{H}$ & 95,264 & 225,679 & 0.59 & 0.95 & 0.03 & 1.69 & 1.15 \\
\hline Thiodicarb & I & 92,466 & 128,697 & 6.21 & 6.55 & 0.03 & 1.64 & 8.62 \\
\hline Metolachlor & $\mathrm{H}$ & 92,366 & 62,331 & 0.16 & 0.2 & 0.03 & 1.64 & 0.34 \\
\hline Cyanazine & $\mathrm{H}$ & 88,332 & 113,860 & 0.3 & 0.72 & 0.03 & 1.57 & 0.64 \\
\hline Oil & I & 85,915 & 5,080 & 0.16 & 0.38 & 0.03 & 1.53 & 0.22 \\
\hline Aldicarb & I & 82,887 & 136,102 & 1.94 & 3.15 & 0.03 & 1.47 & 2.61 \\
\hline Ethephon & OP & 82,279 & 66,741 & 2.67 & 2.27 & 0.03 & 1.46 & 3.5 \\
\hline Profenofos & I & 81,630 & 68,903 & 3.85 & 4.06 & 0.03 & 1.45 & 5.58 \\
\hline Sodium chlorate & OP & 79,162 & 22,977 & 1.51 & 1.34 & 0.03 & 1.41 & 1.96 \\
\hline Norflurazon & $\mathrm{H}$ & 76,713 & 107,203 & 3.38 & 4.47 & 0.03 & 1.36 & 4.08 \\
\hline PCNB & $\mathrm{F}$ & 68,541 & 82,010 & 4.04 & 5.17 & 0.03 & 1.22 & 5.59 \\
\hline Acephate & I & 62,828 & 95,527 & 1.73 & 3 & 0.02 & 1.12 & 3.04 \\
\hline DSMA & $\mathrm{H}$ & 59,679 & 22,395 & 4.28 & 2.92 & 0.02 & 1.06 & 5.4 \\
\hline Butylate & $\mathrm{H}$ & 58,729 & 16,359 & 0.76 & 0.82 & 0.02 & 1.04 & 1.97 \\
\hline Malathion & I & 58,148 & 43,112 & 1.87 & 2.23 & 0.02 & 1.03 & 4.04 \\
\hline \multicolumn{9}{|c|}{ New England Coastal Basins (NECB) } \\
\hline \multicolumn{9}{|c|}{ [Study Unit Area: 14,636,166 acres; Area in Cropland and Pasture: 392,572 acres] } \\
\hline Oil & I & 241,120 & 8,118 & 0.45 & 0.6 & 0.61 & 26.46 & 0.62 \\
\hline Mancozeb & $\mathrm{F}$ & 76,207 & 18,372 & 0.92 & 0.9 & 0.19 & 8.36 & 1.42 \\
\hline Captan & $\mathrm{F}$ & 62,707 & 8,216 & 1.65 & 1.4 & 0.16 & 6.88 & 3.22 \\
\hline Metolachlor & $\mathrm{H}$ & 58,650 & 31,218 & 0.1 & 0.1 & 0.15 & 6.44 & 0.22 \\
\hline Atrazine & $\mathrm{H}$ & 58,558 & 41,171 & 0.09 & 0.07 & 0.15 & 6.43 & 0.21 \\
\hline Chlorothalonil & $\mathrm{F}$ & 43,555 & 10,633 & 0.34 & 0.34 & 0.11 & 4.78 & 0.48 \\
\hline Dichlobenil & $\mathrm{H}$ & 24,504 & 8,271 & 37.96 & 29.82 & 0.06 & 2.69 & 66.59 \\
\hline Metiram & $\mathrm{F}$ & 23,965 & 1,720 & 2.6 & 1.35 & 0.06 & 2.63 & 5.68 \\
\hline Diazinon & I & 23,833 & 9,563 & 1.48 & 0.89 & 0.06 & 2.61 & 2.03 \\
\hline Azinphos methyl & $\mathrm{I}$ & 20,502 & 11,796 & 0.82 & 0.46 & 0.05 & 2.25 & 1.47 \\
\hline Chlorpyrifos & I & 20,251 & 10,802 & 0.13 & 0.08 & 0.05 & 2.22 & 0.25 \\
\hline Maneb & $\mathrm{F}$ & 19,749 & 5,114 & 0.66 & 0.73 & 0.05 & 2.17 & 1.15 \\
\hline Carbaryl & I & 17,775 & 12,557 & 0.39 & 0.43 & 0.05 & 1.95 & 0.76 \\
\hline Copper & $\mathrm{F}$ & 17,362 & 3,990 & 0.17 & 0.16 & 0.04 & 1.9 & 0.2 \\
\hline Napropamide & $\mathrm{H}$ & 16,621 & 4,300 & 3.51 & 1.2 & 0.04 & 1.82 & 5.57 \\
\hline
\end{tabular}


Table 5. Ranking of pesticides used in the National Water-Quality Assessment Program study units during 1992-1995- Continued

\begin{tabular}{|c|c|c|c|c|c|c|c|c|}
\hline Compound & $\begin{array}{c}\text { Pesticide } \\
\text { type }\end{array}$ & $\begin{array}{l}\text { Average } \\
\text { amount } \\
\text { applied } \\
\text { (lb a.i.) }\end{array}$ & $\begin{array}{l}\text { Study unit } \\
\text { area treated } \\
\text { (acres) }\end{array}$ & $\begin{array}{c}\text { Percentage of } \\
\text { national use }\end{array}$ & $\begin{array}{l}\text { Percentage of } \\
\text { U.S. area } \\
\text { treated }\end{array}$ & $\begin{array}{c}\text { Average } \\
\text { amount } \\
\text { applied to } \\
\text { cropland and } \\
\text { pasture area } \\
\text { (lb a.i.) }\end{array}$ & $\begin{array}{l}\text { Percentage of } \\
\text { all reported } \\
\text { pesticides } \\
\text { used in the } \\
\text { study unit }\end{array}$ & $\begin{array}{c}\text { Percentage of } \\
\text { total use } \\
\text { among study } \\
\text { units }\end{array}$ \\
\hline \multicolumn{9}{|c|}{ New England Coastal Basins (NECB)_Continued } \\
\hline Methyl bromide & OP & 16,146 & 96 & 0.04 & 0.05 & 0.04 & 1.77 & 0.06 \\
\hline Propargite & I & 15,176 & 6,641 & 0.44 & 0.34 & 0.04 & 1.67 & 0.7 \\
\hline Ferbam & $\mathrm{F}$ & 13,377 & 2,973 & 5.97 & 4.24 & 0.03 & 1.47 & 10.1 \\
\hline Alachlor & $\mathrm{H}$ & 11,553 & 6,020 & 0.05 & 0.04 & 0.03 & 1.27 & 0.1 \\
\hline Cryolite & I & 11,097 & 667 & 0.36 & 0.14 & 0.03 & 1.22 & 0.52 \\
\hline Cyanazine & $\mathrm{H}$ & 11,054 & 7,075 & 0.04 & 0.05 & 0.03 & 1.21 & 0.08 \\
\hline Dodine & $\mathrm{F}$ & 10,492 & 6,616 & 3.53 & 3.66 & 0.03 & 1.15 & 7.99 \\
\hline \multicolumn{9}{|c|}{ Northern Rockies Intermontane Basins (NROK) } \\
\hline \multicolumn{9}{|c|}{ [Study Unit Area: 20,153,264 acres; Area in Cropland and Pasture: 1,079,780 acres] } \\
\hline 2,4-D & $\mathrm{H}$ & 114,659 & 243,213 & 0.31 & 0.31 & 0.11 & 17.99 & 0.85 \\
\hline EPTC & $\mathrm{H}$ & 58,937 & 20,218 & 0.42 & 0.5 & 0.05 & 9.25 & 0.89 \\
\hline Diuron & $\mathrm{H}$ & 40,867 & 30,308 & 1.09 & 0.93 & 0.04 & 6.41 & 1.51 \\
\hline MCPA & $\mathrm{H}$ & 39,827 & 98,080 & 0.86 & 0.8 & 0.04 & 6.25 & 1.77 \\
\hline DCPA & $\mathrm{H}$ & 31,793 & 3,179 & 3.19 & 1.72 & 0.03 & 4.99 & 9.21 \\
\hline Triallate & $\mathrm{H}$ & 29,247 & 26,687 & 1.28 & 1.2 & 0.03 & 4.59 & 2.92 \\
\hline Oil & I & 23,745 & 663 & 0.05 & 0.05 & 0.02 & 3.73 & 0.06 \\
\hline Bromoxynil & $\mathrm{H}$ & 17,514 & 73,539 & 0.52 & 0.59 & 0.02 & 2.75 & 1.22 \\
\hline Propargite & I & 17,490 & 12,966 & 0.51 & 0.67 & 0.02 & 2.74 & 0.81 \\
\hline Metribuzin & $\mathrm{H}$ & 16,492 & 37,596 & 0.61 & 0.45 & 0.02 & 2.59 & 1 \\
\hline Diclofop & $\mathrm{H}$ & 16,283 & 19,382 & 1.46 & 1.38 & 0.02 & 2.56 & 2.63 \\
\hline Ethalfluralin & $\mathrm{H}$ & 11,942 & 15,367 & 0.49 & 0.5 & 0.01 & 1.87 & 0.83 \\
\hline Trifluralin & $\mathrm{H}$ & 11,588 & 21,719 & 0.06 & 0.09 & 0.01 & 1.82 & 0.12 \\
\hline Glyphosate & $\mathrm{H}$ & 11,467 & 22,520 & 0.07 & 0.1 & 0.01 & 1.8 & 0.14 \\
\hline Chlorpyrifos & I & 11,138 & 12,296 & 0.07 & 0.09 & 0.01 & 1.75 & 0.14 \\
\hline Dicamba & $\mathrm{H}$ & 10,737 & 80,313 & 0.11 & 0.21 & 0.01 & 1.68 & 0.26 \\
\hline Malathion & I & 10,304 & 8,320 & 0.33 & 0.43 & 0.01 & 1.62 & 0.72 \\
\hline Alachlor & $\mathrm{H}$ & 8,855 & 3,841 & 0.04 & 0.03 & 0.01 & 1.39 & 0.08 \\
\hline Dicofol & I & 8,622 & 8,594 & 0.79 & 1.03 & 0.01 & 1.35 & 1.02 \\
\hline Carbaryl & I & 8,157 & 8,029 & 0.18 & 0.27 & 0.01 & 1.28 & 0.35 \\
\hline Carbofuran & I & 8,145 & 30,168 & 0.16 & 0.46 & 0.01 & 1.28 & 0.36 \\
\hline Simazine & $\mathrm{H}$ & 7,517 & 7,642 & 0.16 & 0.22 & 0.01 & 1.18 & 0.25 \\
\hline Benomyl & $\mathrm{F}$ & 7,212 & 13,670 & 0.58 & 0.92 & 0.01 & 1.13 & 0.78 \\
\hline Thiabendazole & $\mathrm{F}$ & 6,752 & 12,277 & 4.24 & 4.24 & 0.01 & 1.06 & 6.77 \\
\hline $2,4-\mathrm{Db}$ & $\mathrm{H}$ & 6,725 & 13,449 & 0.7 & 0.3 & 0.01 & 1.06 & 1.32 \\
\hline Picloram & $\mathrm{H}$ & 6,565 & 27,353 & 0.38 & 0.39 & 0.01 & 1.03 & 1.87 \\
\hline Ethyl parathion & I & 6,548 & 13,909 & 0.32 & 0.41 & 0.01 & 1.03 & 1.22 \\
\hline \multicolumn{9}{|c|}{ Nevada Basin and Range (NVBR) } \\
\hline \multicolumn{9}{|c|}{ [Study Unit Area: 5,660,898 acres; Area in Cropland and Pasture: 70,350 acres] } \\
\hline Oil & I & 23,722 & 435 & 0.05 & 0.03 & 0.34 & 27.12 & 0.06 \\
\hline Sulfur & $\mathrm{F}$ & 16,242 & 461 & 0.02 & 0.02 & 0.23 & 18.57 & 0.03 \\
\hline Copper & $\mathrm{F}$ & 11,729 & 1,369 & 0.11 & 0.06 & 0.17 & 13.41 & 0.14 \\
\hline Molinate & $\mathrm{H}$ & 8,141 & 2,066 & 0.17 & 0.15 & 0.12 & 9.31 & 0.2 \\
\hline
\end{tabular}


Table 5. Ranking of pesticides used in the National W ater-Quality Assessment Program study units during 1992-1995- Continued

\begin{tabular}{|c|c|c|c|c|c|c|c|c|}
\hline Compound & $\begin{array}{c}\text { Pesticide } \\
\text { type }\end{array}$ & $\begin{array}{l}\text { Average } \\
\text { amount } \\
\text { applied } \\
\text { (lb a.i.) }\end{array}$ & $\begin{array}{l}\text { Study unit } \\
\text { area treated } \\
\text { (acres) }\end{array}$ & $\begin{array}{l}\text { Percentage of } \\
\text { national use }\end{array}$ & $\begin{array}{l}\text { Percentage of } \\
\text { U.S. area } \\
\text { treated }\end{array}$ & $\begin{array}{c}\text { Average } \\
\text { amount } \\
\text { applied to } \\
\text { cropland and } \\
\text { pasture area } \\
\text { (lb a.i.) }\end{array}$ & $\begin{array}{l}\text { Percentage of } \\
\text { all reported } \\
\text { pesticides } \\
\text { used in the } \\
\text { study unit }\end{array}$ & $\begin{array}{c}\text { Percentage of } \\
\text { total use } \\
\text { among study } \\
\text { units }\end{array}$ \\
\hline \multicolumn{9}{|c|}{ Nevada Basin and Range (NVBR)—Continued } \\
\hline Methyl bromide & $\mathrm{OP}$ & 2,636 & 22 & 0.01 & 0.01 & 0.04 & 3.01 & 0.01 \\
\hline Hexazinone & $\mathrm{H}$ & 2,455 & 2,469 & 0.53 & 0.42 & 0.03 & 2.81 & 1.54 \\
\hline 2,4-Db & $\mathrm{H}$ & 2,184 & 4,335 & 0.23 & 0.1 & 0.03 & 2.5 & 0.43 \\
\hline $2,4-\mathrm{D}$ & $\mathrm{H}$ & 1,425 & 1,714 & 0 & 0 & 0.02 & 1.63 & 0.01 \\
\hline Thiobencarb & $\mathrm{H}$ & 1,371 & 341 & 0.1 & 0.07 & 0.02 & 1.57 & 0.15 \\
\hline Carbofuran & I & 1,281 & 2,199 & 0.03 & 0.03 & 0.02 & 1.46 & 0.06 \\
\hline Metribuzin & $\mathrm{H}$ & 1,049 & 1,747 & 0.04 & 0.02 & 0.01 & 1.2 & 0.06 \\
\hline Glyphosate & $\mathrm{H}$ & 1,013 & 1,482 & 0.01 & 0.01 & 0.01 & 1.16 & 0.01 \\
\hline DCPA & $\mathrm{H}$ & 1,007 & 134 & 0.1 & 0.07 & 0.01 & 1.15 & 0.29 \\
\hline Propargite & $\mathrm{I}$ & 893 & 535 & 0.03 & 0.03 & 0.01 & 1.02 & 0.04 \\
\hline \multicolumn{9}{|c|}{ Ozark Plateaus (OZRK) } \\
\hline \multicolumn{9}{|c|}{ [Study Unit Area: 30,471,632 acres; Area in Cropland and Pasture: 8,310,109 acres] } \\
\hline $2,4-\mathrm{D}$ & $\mathrm{H}$ & 619,950 & $1,199,373$ & 1.67 & 1.54 & 0.07 & 14.42 & 4.62 \\
\hline Propanil & $\mathrm{H}$ & 556,285 & 145,725 & 7.69 & 6.23 & 0.07 & 12.94 & 9.57 \\
\hline Atrazine & $\mathrm{H}$ & 542,124 & 427,806 & 0.85 & 0.75 & 0.07 & 12.61 & 1.93 \\
\hline Metolachlor & $\mathrm{H}$ & 369,986 & 217,370 & 0.64 & 0.7 & 0.04 & 8.61 & 1.36 \\
\hline Glyphosate & $\mathrm{H}$ & 274,266 & 615,461 & 1.71 & 2.58 & 0.03 & 6.38 & 3.3 \\
\hline Alachlor & $\mathrm{H}$ & 215,606 & 125,416 & 0.84 & 0.86 & 0.03 & 5.01 & 1.89 \\
\hline Trifluralin & $\mathrm{H}$ & 191,382 & 236,577 & 0.97 & 0.93 & 0.02 & 4.45 & 1.95 \\
\hline Pendimethalin & $\mathrm{H}$ & 173,081 & 208,896 & 0.84 & 0.97 & 0.02 & 4.03 & 1.61 \\
\hline Molinate & $\mathrm{H}$ & 111,672 & 33,590 & 2.28 & 2.37 & 0.01 & 2.6 & 2.75 \\
\hline Dicamba & $\mathrm{H}$ & 100,433 & 615,070 & 1.05 & 1.58 & 0.01 & 2.34 & 2.47 \\
\hline Thiobencarb & $\mathrm{H}$ & 89,660 & 25,580 & 6.24 & 5.41 & 0.01 & 2.09 & 9.64 \\
\hline Carbofuran & I & 86,950 & 115,529 & 1.7 & 1.77 & 0.01 & 2.02 & 3.84 \\
\hline Cyanazine & $\mathrm{H}$ & 84,668 & 45,723 & 0.29 & 0.29 & 0.01 & 1.97 & 0.61 \\
\hline Propachlor & $\mathrm{H}$ & 76,810 & 25,042 & 1.99 & 1.5 & 0.01 & 1.79 & 5.84 \\
\hline Chlorpyrifos & I & 58,369 & 84,689 & 0.38 & 0.61 & 0.01 & 1.36 & 0.73 \\
\hline Bentazon & $\mathrm{H}$ & 53,372 & 93,053 & 0.79 & 0.93 & 0.01 & 1.24 & 1.37 \\
\hline Butylate & $\mathrm{H}$ & 44,712 & 10,505 & 0.58 & 0.53 & 0.01 & 1.04 & 1.5 \\
\hline \multicolumn{9}{|c|}{ Potomac River Basin (POTO) } \\
\hline \multicolumn{9}{|c|}{ [Study Unit Area: 9,065,738 acres; Area in Cropland and Pasture: 1,794,987 acres] } \\
\hline Oil & I & 913,982 & 28,739 & 1.72 & 2.12 & 0.51 & 21.21 & 2.37 \\
\hline Sulfur & $\mathrm{F}$ & 457,252 & 15,129 & 0.53 & 0.61 & 0.25 & 10.61 & 0.75 \\
\hline Metolachlor & $\mathrm{H}$ & 417,324 & 220,804 & 0.72 & 0.71 & 0.23 & 9.68 & 1.53 \\
\hline Atrazine & $\mathrm{H}$ & 377,874 & 279,091 & 0.59 & 0.49 & 0.21 & 8.77 & 1.35 \\
\hline Captan & $\mathrm{F}$ & 255,168 & 33,669 & 6.71 & 5.75 & 0.14 & 5.92 & 13.1 \\
\hline Alachlor & $\mathrm{H}$ & 203,602 & 108,850 & 0.79 & 0.75 & 0.11 & 4.72 & 1.78 \\
\hline $2,4-\mathrm{D}$ & $\mathrm{H}$ & 161,023 & 274,636 & 0.43 & 0.35 & 0.09 & 3.74 & 1.2 \\
\hline Ziram & $\mathrm{F}$ & 131,545 & 17,158 & 4.77 & 3.48 & 0.07 & 3.05 & 6.09 \\
\hline Mancozeb & $\mathrm{F}$ & 128,363 & 25,509 & 1.55 & 1.25 & 0.07 & 2.98 & 2.39 \\
\hline Simazine & $\mathrm{H}$ & 94,422 & 88,937 & 1.96 & 2.59 & 0.05 & 2.19 & 3.08 \\
\hline Chlorpyrifos & I & 82,355 & 71,128 & 0.53 & 0.51 & 0.05 & 1.91 & 1.02 \\
\hline
\end{tabular}


Table 5. Ranking of pesticides used in the National W ater-Quality Assessment Program study units during 1992-1995- Continued

\begin{tabular}{|c|c|c|c|c|c|c|c|c|}
\hline Compound & $\begin{array}{c}\text { Pesticide } \\
\text { type }\end{array}$ & $\begin{array}{l}\text { Average } \\
\text { amount } \\
\text { applied } \\
\text { (lb a.i.) }\end{array}$ & $\begin{array}{l}\text { Study unit } \\
\text { area treated } \\
\quad(\text { acres })\end{array}$ & $\begin{array}{l}\text { Percentage of } \\
\text { national use }\end{array}$ & $\begin{array}{l}\text { Percentage of } \\
\text { U.S. area } \\
\text { treated }\end{array}$ & $\begin{array}{c}\text { Average } \\
\text { amount } \\
\text { applied to } \\
\text { cropland and } \\
\text { pasture area } \\
\text { (lb a.i.) } \\
\end{array}$ & $\begin{array}{l}\text { Percentage of } \\
\text { all reported } \\
\text { pesticides } \\
\text { used in the } \\
\text { study unit }\end{array}$ & $\begin{array}{c}\text { Percentage of } \\
\text { total use } \\
\text { among study } \\
\text { units }\end{array}$ \\
\hline \multicolumn{9}{|c|}{ Potomac River Basin (POTO)_Continued } \\
\hline Azinphos methyl & I & 71,786 & 35,921 & 2.88 & 1.39 & 0.04 & 1.67 & 5.13 \\
\hline Metiram & $\mathrm{F}$ & 71,432 & 6,983 & 7.75 & 5.49 & 0.04 & 1.66 & 16.94 \\
\hline Pendimethalin & $\mathrm{H}$ & 58,785 & 55,920 & 0.28 & 0.26 & 0.03 & 1.36 & 0.55 \\
\hline Glyphosate & $\mathrm{H}$ & 52,704 & 58,955 & 0.33 & 0.25 & 0.03 & 1.22 & 0.63 \\
\hline Paraquat & $\mathrm{H}$ & 50,465 & 106,131 & 1.19 & 1.12 & 0.03 & 1.17 & 2.14 \\
\hline Cyanazine & $\mathrm{H}$ & 47,083 & 29,919 & 0.16 & 0.19 & 0.03 & 1.09 & 0.34 \\
\hline Methyl bromide & $\mathrm{OP}$ & 46,295 & 165 & 0.12 & 0.08 & 0.03 & 1.07 & 0.17 \\
\hline Carbofuran & I & 45,567 & 50,606 & 0.89 & 0.78 & 0.03 & 1.06 & 2.01 \\
\hline Methomyl & I & 44,809 & 45,938 & 1.83 & 1.35 & 0.02 & 1.04 & 3.07 \\
\hline \multicolumn{9}{|c|}{ Puget Sound Drainages (PUGT) } \\
\hline \multicolumn{9}{|c|}{ [Study Unit Area: 8,418,539 acres; Area in Cropland and Pasture: 305,796 acres] } \\
\hline Metam sodium & OP & 701,062 & 4,542 & 2.18 & 1.18 & 2.29 & 42.61 & 2.86 \\
\hline $1,3-\mathrm{D}$ & $\mathrm{OP}$ & 360,247 & 2,189 & 0.9 & 0.44 & 1.18 & 21.9 & 1.47 \\
\hline Sulfuric acid & $\mathrm{OP}$ & 135,591 & 661 & 0.52 & 0.63 & 0.44 & 8.24 & 0.62 \\
\hline Oil & I & 45,895 & 1,718 & 0.09 & 0.13 & 0.15 & 2.79 & 0.12 \\
\hline Sulfur & $\mathrm{F}$ & 33,092 & 4,155 & 0.04 & 0.17 & 0.11 & 2.01 & 0.05 \\
\hline Chlorothalonil & $\mathrm{F}$ & 29,309 & 7,549 & 0.23 & 0.24 & 0.1 & 1.78 & 0.32 \\
\hline EPTC & $\mathrm{H}$ & 28,411 & 8,185 & 0.2 & 0.2 & 0.09 & 1.73 & 0.43 \\
\hline Chloropicrin & $\mathrm{OP}$ & 26,666 & 525 & 0.36 & 0.43 & 0.09 & 1.62 & 0.62 \\
\hline $2,4-\mathrm{D}$ & $\mathrm{H}$ & 25,338 & 36,437 & 0.07 & 0.05 & 0.08 & 1.54 & 0.19 \\
\hline Captan & $\mathrm{F}$ & 23,188 & 5,279 & 0.61 & 0.9 & 0.08 & 1.41 & 1.19 \\
\hline \multicolumn{9}{|c|}{ Red River of the North (REDN) } \\
\hline \multicolumn{9}{|c|}{ Study Unit Area: 22,404,260 acres; Area in Cropland and Pasture: 12,336,646 acres] } \\
\hline Sulfuric acid & OP & $1,926,030$ & 13,564 & 7.43 & 12.84 & 0.16 & 13.59 & 8.87 \\
\hline MCPA & $\mathrm{H}$ & $1,227,234$ & $3,478,319$ & 26.34 & 28.24 & 0.1 & 8.66 & 54.45 \\
\hline $2,4-\mathrm{D}$ & $\mathrm{H}$ & $1,208,129$ & $3,636,655$ & 3.24 & 4.68 & 0.1 & 8.52 & 9 \\
\hline Trifluralin & $\mathrm{H}$ & $1,069,687$ & $1,689,610$ & 5.41 & 6.66 & 0.09 & 7.55 & 10.9 \\
\hline EPTC & $\mathrm{H}$ & 817,950 & 237,990 & 5.8 & 5.93 & 0.07 & 5.77 & 12.4 \\
\hline Chlorothalonil & $\mathrm{F}$ & 618,234 & 157,985 & 4.83 & 5.04 & 0.05 & 4.36 & 6.84 \\
\hline Ethalfluralin & $\mathrm{H}$ & 516,193 & 635,563 & 20.98 & 20.61 & 0.04 & 3.64 & 35.86 \\
\hline Triallate & $\mathrm{H}$ & 492,751 & 488,437 & 21.58 & 21.96 & 0.04 & 3.48 & 49.21 \\
\hline Mancozeb & $\mathrm{F}$ & 467,679 & 171,923 & 5.63 & 8.41 & 0.04 & 3.3 & 8.72 \\
\hline Bentazon & $\mathrm{H}$ & 407,354 & 532,886 & 6.05 & 5.32 & 0.03 & 2.87 & 10.49 \\
\hline Sodium chlorate & $\mathrm{OP}$ & 389,256 & 129,752 & 7.42 & 7.58 & 0.03 & 2.75 & 9.61 \\
\hline Metolachlor & $\mathrm{H}$ & 351,605 & 169,076 & 0.61 & 0.54 & 0.03 & 2.48 & 1.29 \\
\hline Bromoxynil & $\mathrm{H}$ & 345,419 & $1,302,867$ & 10.22 & 10.52 & 0.03 & 2.44 & 24.02 \\
\hline Pendimethalin & $\mathrm{H}$ & 290,515 & 229,502 & 1.4 & 1.07 & 0.02 & 2.05 & 2.71 \\
\hline Alachlor & $\mathrm{H}$ & 279,015 & 138,012 & 1.09 & 0.95 & 0.02 & 1.97 & 2.44 \\
\hline Dicamba & $\mathrm{H}$ & 269,755 & $2,004,067$ & 2.81 & 5.14 & 0.02 & 1.9 & 6.63 \\
\hline Terbufos & I & 243,581 & 239,734 & 3.38 & 3.59 & 0.02 & 1.72 & 7.9 \\
\hline Cycloate & $\mathrm{H}$ & 219,707 & 73,236 & 23.73 & 20.15 & 0.02 & 1.55 & 37.11 \\
\hline Acetochlor & $\mathrm{H}$ & 211,697 & 110,629 & 0.89 & 0.93 & 0.02 & 1.49 & 1.91 \\
\hline
\end{tabular}


Table 5. Ranking of pesticides used in the National W ater-Quality Assessment Program study units during 1992-1995- Continued

\begin{tabular}{|c|c|c|c|c|c|c|c|c|}
\hline Compound & $\begin{array}{c}\text { Pesticide } \\
\text { type }\end{array}$ & $\begin{array}{l}\text { Average } \\
\text { amount } \\
\text { applied } \\
\text { (lb a.i.) }\end{array}$ & $\begin{array}{l}\text { Study unit } \\
\text { area treated } \\
\text { (acres) }\end{array}$ & $\begin{array}{l}\text { Percentage of } \\
\text { national use }\end{array}$ & $\begin{array}{l}\text { Percentage of } \\
\text { U.S. area } \\
\text { treated }\end{array}$ & $\begin{array}{c}\text { Average } \\
\text { amount } \\
\text { applied to } \\
\text { cropland and } \\
\text { pasture area } \\
\text { (lb a.i.) }\end{array}$ & $\begin{array}{l}\text { Percentage of } \\
\text { all reported } \\
\text { pesticides } \\
\text { used in the } \\
\text { study unit }\end{array}$ & $\begin{array}{c}\text { Percentage of } \\
\text { total use } \\
\text { among study } \\
\text { units }\end{array}$ \\
\hline \multicolumn{9}{|c|}{ Red River of the North (REDN)—Continued } \\
\hline Chlorpyrifos & I & 209,552 & 169,674 & 1.36 & 1.22 & 0.02 & 1.48 & 2.6 \\
\hline Diclofop & $\mathrm{H}$ & 207,761 & 258,520 & 18.59 & 18.37 & 0.02 & 1.47 & 33.53 \\
\hline Imazamethabenz & $\mathrm{H}$ & 189,351 & 573,390 & 42.09 & 39.35 & 0.02 & 1.34 & 72.99 \\
\hline Cyanazine & $\mathrm{H}$ & 148,423 & 105,845 & 0.5 & 0.67 & 0.01 & 1.05 & 1.07 \\
\hline \multicolumn{9}{|c|}{ Rio Grande Valley (RIOG) } \\
\hline \multicolumn{9}{|c|}{ [Study Unit Area: 29,381,958 acres; Area in Cropland and Pasture: 683,138 acres] } \\
\hline Sulfuric acid & $\mathrm{OP}$ & $1,583,174$ & 4,645 & 6.1 & 4.4 & 2.32 & 57.28 & 7.29 \\
\hline $2,4-\mathrm{D}$ & $\mathrm{H}$ & 317,785 & 638,334 & 0.85 & 0.82 & 0.47 & 11.5 & 2.37 \\
\hline EPTC & $\mathrm{H}$ & 189,823 & 47,306 & 1.35 & 1.18 & 0.28 & 6.87 & 2.88 \\
\hline Mancozeb & $\mathrm{F}$ & 54,291 & 14,809 & 0.65 & 0.73 & 0.08 & 1.96 & 1.01 \\
\hline Ethoprop & I & 47,993 & 11,033 & 3.78 & 3.28 & 0.07 & 1.74 & 5.39 \\
\hline Chlorothalonil & $\mathrm{F}$ & 47,301 & 26,167 & 0.37 & 0.83 & 0.07 & 1.71 & 0.52 \\
\hline Sodium chlorate & $\mathrm{OP}$ & 46,797 & 10,301 & 0.89 & 0.6 & 0.07 & 1.69 & 1.16 \\
\hline Picloram & $\mathrm{H}$ & 45,926 & 191,331 & 2.67 & 2.69 & 0.07 & 1.66 & 13.11 \\
\hline DCPA & $\mathrm{H}$ & 39,110 & 5,494 & 3.92 & 2.97 & 0.06 & 1.42 & 11.33 \\
\hline Trifluralin & $\mathrm{H}$ & 32,261 & 22,663 & 0.16 & 0.09 & 0.05 & 1.17 & 0.33 \\
\hline $1,3-\mathrm{D}$ & $\mathrm{OP}$ & 32,127 & 1,068 & 0.08 & 0.22 & 0.05 & 1.16 & 0.13 \\
\hline Endosulfan & I & 30,205 & 20,280 & 1.45 & 1.26 & 0.04 & 1.09 & 2.24 \\
\hline Chlorpyrifos & I & 28,034 & 37,474 & 0.18 & 0.27 & 0.04 & 1.01 & 0.35 \\
\hline \multicolumn{9}{|c|}{ Sacramento Basin (SACR) } \\
\hline \multicolumn{9}{|c|}{ [Study Unit Area: 17,154,866 acres; Area in Cropland and Pasture: 1,880,360 acres] } \\
\hline Sulfur & $\mathrm{F}$ & $6,832,764$ & 179,435 & 7.88 & 7.26 & 3.63 & 30.96 & 11.23 \\
\hline Oil & I & $4,751,392$ & 125,370 & 8.93 & 9.25 & 2.53 & 21.53 & 12.3 \\
\hline Copper & $\mathrm{F}$ & $2,713,644$ & 371,565 & 25.77 & 14.83 & 1.44 & 12.3 & 31.14 \\
\hline Molinate & $\mathrm{H}$ & $1,438,632$ & 365,135 & 29.34 & 25.71 & 0.77 & 6.52 & 35.41 \\
\hline Methyl bromide & OP & 994,167 & 11,072 & 2.53 & 5.42 & 0.53 & 4.51 & 3.54 \\
\hline Metam sodium & OP & 940,354 & 25,128 & 2.92 & 6.55 & 0.5 & 4.26 & 3.84 \\
\hline Ziram & $\mathrm{F}$ & 366,616 & 67,845 & 13.28 & 13.77 & 0.19 & 1.66 & 16.98 \\
\hline Glyphosate & $\mathrm{H}$ & 272,073 & 314,464 & 1.7 & 1.32 & 0.14 & 1.23 & 3.27 \\
\hline Diazinon & I & 243,434 & 134,657 & 15.07 & 12.52 & 0.13 & 1.1 & 20.69 \\
\hline Thiobencarb & $\mathrm{H}$ & 242,239 & 60,228 & 16.87 & 12.73 & 0.13 & 1.1 & 26.04 \\
\hline Chlorpyrifos & I & 230,418 & 161,260 & 1.5 & 1.16 & 0.12 & 1.04 & 2.86 \\
\hline \multicolumn{9}{|c|}{ Santa Ana Basin (SANA) } \\
\hline \multicolumn{9}{|c|}{ [Study Unit Area: 1,705,405 acres; Area in Cropland and Pasture: 51,627 acres] } \\
\hline Sulfur & $\mathrm{F}$ & 422,944 & 6,685 & 0.49 & 0.27 & 8.19 & 36.45 & 0.7 \\
\hline Oil & I & 207,038 & 3,405 & 0.39 & 0.25 & 4.01 & 17.84 & 0.54 \\
\hline Methyl bromide & $\mathrm{OP}$ & 190,832 & 888 & 0.49 & 0.43 & 3.7 & 16.45 & 0.68 \\
\hline Chloropicrin & OP & 79,137 & 801 & 1.06 & 0.65 & 1.53 & 6.82 & 1.84 \\
\hline Copper & $\mathrm{F}$ & 24,576 & 7,637 & 0.23 & 0.31 & 0.48 & 2.12 & 0.28 \\
\hline Chlorpyrifos & I & 19,439 & 9,558 & 0.13 & 0.07 & 0.38 & 1.68 & 0.24 \\
\hline Glyphosate & $\mathrm{H}$ & 18,678 & 16,648 & 0.12 & 0.07 & 0.36 & 1.61 & 0.23 \\
\hline
\end{tabular}


Table 5. Ranking of pesticides used in the National W ater-Quality Assessment Program study units during 1992-1995- Continued

\begin{tabular}{|c|c|c|c|c|c|c|c|c|}
\hline Compound & $\begin{array}{c}\text { Pesticide } \\
\text { type }\end{array}$ & $\begin{array}{l}\text { Average } \\
\text { amount } \\
\text { applied } \\
\text { (lb a.i.) }\end{array}$ & $\begin{array}{l}\text { Study unit } \\
\text { area treated } \\
\text { (acres) }\end{array}$ & $\begin{array}{l}\text { Percentage of } \\
\text { national use }\end{array}$ & $\begin{array}{l}\text { Percentage of } \\
\text { U.S. area } \\
\text { treated }\end{array}$ & $\begin{array}{c}\text { Average } \\
\text { amount } \\
\text { applied to } \\
\text { cropland and } \\
\text { pasture area } \\
\text { (lb a.i.) } \\
\end{array}$ & $\begin{array}{l}\text { Percentage of } \\
\text { all reported } \\
\text { pesticides } \\
\text { used in the } \\
\text { study unit }\end{array}$ & $\begin{array}{c}\text { Percentage of } \\
\text { total use } \\
\text { among study } \\
\text { units }\end{array}$ \\
\hline \multicolumn{9}{|c|}{ Santa Ana Basin (SANA)_Continued } \\
\hline Metam sodium & $\mathrm{OP}$ & 17,352 & 221 & 0.05 & 0.06 & 0.34 & 1.5 & 0.07 \\
\hline Cryolite & I & 15,656 & 2,551 & 0.51 & 0.53 & 0.3 & 1.35 & 0.74 \\
\hline Carbaryl & I & 12,349 & 1,959 & 0.27 & 0.07 & 0.24 & 1.06 & 0.53 \\
\hline Simazine & $\mathrm{H}$ & 11,633 & 6,054 & 0.24 & 0.18 & 0.23 & 1 & 0.38 \\
\hline \multicolumn{9}{|c|}{ San Joaquin-Tulare (SANJ) } \\
\hline \multicolumn{9}{|c|}{ [Study Unit Area: 19,966,328 acres; Area in Cropland and Pasture: 4,207,813 acres] } \\
\hline Sulfur & $\mathrm{F}$ & $42,154,237$ & 795,288 & 48.59 & 32.18 & 10.02 & 48.49 & 69.27 \\
\hline Oil & I & $12,096,055$ & 357,814 & 22.74 & 26.41 & 2.87 & 13.91 & 31.31 \\
\hline Methyl bromide & OP & $5,149,392$ & 37,156 & 13.12 & 18.18 & 1.22 & 5.92 & 18.34 \\
\hline Metam sodium & OP & $3,112,316$ & 68,151 & 9.65 & 17.75 & 0.74 & 3.58 & 12.7 \\
\hline Copper & $\mathrm{F}$ & $2,819,839$ & 681,123 & 26.78 & 27.18 & 0.67 & 3.24 & 32.36 \\
\hline Cryolite & I & $1,784,366$ & 301,968 & 57.99 & 62.98 & 0.42 & 2.05 & 84.27 \\
\hline Sodium chlorate & $\mathrm{OP}$ & $1,693,511$ & 588,609 & 32.3 & 34.38 & 0.4 & 1.95 & 41.81 \\
\hline Chlorpyrifos & I & $1,479,897$ & 965,403 & 9.6 & 6.92 & 0.35 & 1.7 & 18.38 \\
\hline Glyphosate & $\mathrm{H}$ & $1,282,279$ & $1,405,979$ & 7.99 & 5.9 & 0.3 & 1.48 & 15.42 \\
\hline Ziram & $\mathrm{F}$ & $1,221,458$ & 223,434 & 44.25 & 45.35 & 0.29 & 1.41 & 56.58 \\
\hline Propargite & I & $1,130,815$ & 642,696 & 32.93 & 33.12 & 0.27 & 1.3 & 52.43 \\
\hline \multicolumn{9}{|c|}{ Santee Basin and Coastal Drainage (SANT) } \\
\hline \multicolumn{9}{|c|}{ [Study Unit Area: 14,907,489 acres; Area in Cropland and Pasture: 1,336,276 acres] } \\
\hline Methyl bromide & OP & $1,629,975$ & 7,513 & 4.15 & 3.68 & 1.22 & 26.25 & 5.81 \\
\hline Sulfur & $\mathrm{F}$ & $1,243,081$ & 21,039 & 1.43 & 0.85 & 0.93 & 20.02 & 2.04 \\
\hline $2,4-\mathrm{D}$ & $\mathrm{H}$ & 246,250 & 411,540 & 0.66 & 0.53 & 0.18 & 3.97 & 1.83 \\
\hline Captan & $\mathrm{F}$ & 235,001 & 19,776 & 6.18 & 3.38 & 0.18 & 3.78 & 12.07 \\
\hline Atrazine & $\mathrm{H}$ & 225,911 & 172,334 & 0.35 & 0.3 & 0.17 & 3.64 & 0.8 \\
\hline Oil & I & 220,621 & 6,516 & 0.42 & 0.48 & 0.17 & 3.55 & 0.57 \\
\hline Chloropicrin & $\mathrm{OP}$ & 165,509 & 2,635 & 2.22 & 2.15 & 0.12 & 2.67 & 3.85 \\
\hline $1,3-\mathrm{D}$ & $\mathrm{OP}$ & 152,916 & 3,965 & 0.38 & 0.8 & 0.11 & 2.46 & 0.62 \\
\hline Alachlor & $\mathrm{H}$ & 144,681 & 94,807 & 0.56 & 0.65 & 0.11 & 2.33 & 1.27 \\
\hline Mancozeb & $\mathrm{F}$ & 139,913 & 11,944 & 1.69 & 0.58 & 0.1 & 2.25 & 2.61 \\
\hline Chlorothalonil & $\mathrm{F}$ & 136,117 & 26,592 & 1.06 & 0.85 & 0.1 & 2.19 & 1.51 \\
\hline Metolachlor & $\mathrm{H}$ & 114,274 & 81,309 & 0.2 & 0.26 & 0.09 & 1.84 & 0.42 \\
\hline MSMA & $\mathrm{H}$ & 84,873 & 60,969 & 1.47 & 1.6 & 0.06 & 1.37 & 1.81 \\
\hline Carbaryl & I & 83,650 & 56,312 & 1.84 & 1.91 & 0.06 & 1.35 & 3.58 \\
\hline Chlorpyrifos & I & 82,465 & 59,841 & 0.54 & 0.43 & 0.06 & 1.33 & 1.02 \\
\hline Ziram & $\mathrm{F}$ & 72,908 & 6,812 & 2.64 & 1.38 & 0.05 & 1.17 & 3.38 \\
\hline Aldicarb & I & 64,704 & 60,016 & 1.51 & 1.39 & 0.05 & 1.04 & 2.04 \\
\hline \multicolumn{9}{|c|}{ South Central Texas (SCTX) } \\
\hline \multicolumn{9}{|c|}{ [Study Unit Area: 19,384,346 acres; Area in Cropland and Pasture: 2,983,850 acres] } \\
\hline $2,4-\mathrm{D}$ & $\mathrm{H}$ & 478,345 & 854,308 & 1.29 & 1.1 & 0.16 & 16.88 & 3.56 \\
\hline Atrazine & $\mathrm{H}$ & 368,535 & 449,834 & 0.58 & 0.79 & 0.12 & 13 & 1.31 \\
\hline Metolachlor & $\mathrm{H}$ & 200,622 & 166,262 & 0.35 & 0.53 & 0.07 & 7.08 & 0.74 \\
\hline
\end{tabular}


Table 5. Ranking of pesticides used in the National W ater-Quality Assessment Program study units during 1992-1995- Continued

\begin{tabular}{|c|c|c|c|c|c|c|c|c|}
\hline Compound & $\begin{array}{c}\text { Pesticide } \\
\text { type }\end{array}$ & $\begin{array}{l}\text { Average } \\
\text { amount } \\
\text { applied } \\
\text { (lb a.i.) }\end{array}$ & $\begin{array}{l}\text { Study unit } \\
\text { area treated } \\
\text { (acres) }\end{array}$ & $\begin{array}{l}\text { Percentage of } \\
\text { national use }\end{array}$ & $\begin{array}{c}\text { Percentage of } \\
\text { U.S. area } \\
\text { treated }\end{array}$ & $\begin{array}{c}\text { Average } \\
\text { amount } \\
\text { applied to } \\
\text { cropland and } \\
\text { pasture area } \\
\text { (lb a.i.) }\end{array}$ & $\begin{array}{l}\text { Percentage of } \\
\text { all reported } \\
\text { pesticides } \\
\text { used in the } \\
\text { study unit }\end{array}$ & $\begin{array}{c}\text { Percentage of } \\
\text { total use } \\
\text { among study } \\
\text { units }\end{array}$ \\
\hline \multicolumn{9}{|c|}{ South Central Texas (SCTX)—Continued } \\
\hline $1,3-\mathrm{D}$ & $\mathrm{OP}$ & 149,862 & 2,855 & 0.37 & 0.58 & 0.05 & 5.29 & 0.61 \\
\hline Trifluralin & $\mathrm{H}$ & 134,100 & 187,703 & 0.68 & 0.74 & 0.04 & 4.73 & 1.37 \\
\hline Chlorothalonil & $\mathrm{F}$ & 86,227 & 40,729 & 0.67 & 1.3 & 0.03 & 3.04 & 0.95 \\
\hline Picloram & $\mathrm{H}$ & 76,806 & 286,454 & 4.46 & 4.03 & 0.03 & 2.71 & 21.93 \\
\hline Terbufos & I & 76,683 & 90,374 & 1.07 & 1.35 & 0.03 & 2.71 & 2.49 \\
\hline Chlorpyrifos & I & 72,678 & 84,087 & 0.47 & 0.6 & 0.02 & 2.56 & 0.9 \\
\hline Carbaryl & I & 69,245 & 42,065 & 1.52 & 1.42 & 0.02 & 2.44 & 2.97 \\
\hline Alachlor & $\mathrm{H}$ & 67,311 & 47,273 & 0.26 & 0.33 & 0.02 & 2.38 & 0.59 \\
\hline Pendimethalin & $\mathrm{H}$ & 54,617 & 83,902 & 0.26 & 0.39 & 0.02 & 1.93 & 0.51 \\
\hline Cyanazine & $\mathrm{H}$ & 54,290 & 24,556 & 0.18 & 0.16 & 0.02 & 1.92 & 0.39 \\
\hline Methyl parathion & I & 46,955 & 38,642 & 0.53 & 0.52 & 0.02 & 1.66 & 0.85 \\
\hline Dimethoate & I & 39,473 & 83,031 & 1.16 & 1.61 & 0.01 & 1.39 & 3.57 \\
\hline Malathion & I & 39,305 & 17,845 & 1.27 & 0.92 & 0.01 & 1.39 & 2.73 \\
\hline PCNB & $\mathrm{F}$ & 38,844 & 11,022 & 2.29 & 0.7 & 0.01 & 1.37 & 3.17 \\
\hline Propargite & I & 37,492 & 24,333 & 1.09 & 1.25 & 0.01 & 1.32 & 1.74 \\
\hline Prometryn & $\mathrm{H}$ & 33,758 & 45,619 & 1.83 & 1.78 & 0.01 & 1.19 & 3.27 \\
\hline Glyphosate & $\mathrm{H}$ & 32,326 & 44,482 & 0.2 & 0.19 & 0.01 & 1.14 & 0.39 \\
\hline Butylate & $\mathrm{H}$ & 30,884 & 7,721 & 0.4 & 0.39 & 0.01 & 1.09 & 1.04 \\
\hline \multicolumn{9}{|c|}{ Southern Florida (SOFL) } \\
\hline \multicolumn{9}{|c|}{ [Study Unit Area: 12,537,358 acres; Area in Cropland and Pasture: $1,667,583$ acres] } \\
\hline Methyl bromide & $\mathrm{OP}$ & $10,876,215$ & 59,275 & 27.71 & 29.01 & 6.52 & 31.54 & 38.74 \\
\hline Oil & I & $7,747,359$ & 151,084 & 14.57 & 11.15 & 4.65 & 22.46 & 20.06 \\
\hline Chloropicrin & OP & $2,269,960$ & 35,060 & 30.5 & 28.58 & 1.36 & 6.58 & 52.82 \\
\hline Copper & $\mathrm{F}$ & $1,739,324$ & 240,319 & 16.52 & 9.59 & 1.04 & 5.04 & 19.96 \\
\hline Sulfur & $\mathrm{F}$ & $1,427,677$ & 61,273 & 1.65 & 2.48 & 0.86 & 4.14 & 2.35 \\
\hline Atrazine & $\mathrm{H}$ & $1,330,634$ & 464,739 & 2.08 & 0.82 & 0.8 & 3.86 & 4.74 \\
\hline Mancozeb & $\mathrm{F}$ & $1,179,966$ & 106,743 & 14.21 & 5.22 & 0.71 & 3.42 & 22 \\
\hline Chlorothalonil & $\mathrm{F}$ & 860,835 & 96,961 & 6.72 & 3.09 & 0.52 & 2.5 & 9.53 \\
\hline Bromacil & $\mathrm{H}$ & 611,010 & 196,466 & 51.14 & 44.63 & 0.37 & 1.77 & 55.4 \\
\hline Asulam & $\mathrm{H}$ & 597,438 & 276,037 & 82.86 & 82.6 & 0.36 & 1.73 & 83.67 \\
\hline Simazine & $\mathrm{H}$ & 485,534 & 119,592 & 10.09 & 3.49 & 0.29 & 1.41 & 15.82 \\
\hline Ethion & I & 471,991 & 126,449 & 54.36 & 50.75 & 0.28 & 1.37 & 57.46 \\
\hline Glyphosate & $\mathrm{H}$ & 440,713 & 204,899 & 2.75 & 0.86 & 0.26 & 1.28 & 5.3 \\
\hline Diuron & $\mathrm{H}$ & 435,766 & 193,662 & 11.63 & 5.96 & 0.26 & 1.26 & 16.1 \\
\hline Maneb & $\mathrm{F}$ & 393,555 & 47,068 & 13.08 & 6.7 & 0.24 & 1.14 & 22.91 \\
\hline Phorate & I & 352,458 & 99,531 & 9.25 & 3.31 & 0.21 & 1.02 & 16.11 \\
\hline \multicolumn{9}{|c|}{ Trinity River Basin (TRIN) } \\
\hline \multicolumn{9}{|c|}{ [Study Unit Area: 11,872,636 acres; Area in Cropland and Pasture: 2,689,808 acres] } \\
\hline $2,4-\mathrm{D}$ & $\mathrm{H}$ & 326,427 & 477,953 & 0.88 & 0.61 & 0.12 & 19.17 & 2.43 \\
\hline Propanil & $\mathrm{H}$ & 142,802 & 45,770 & 1.98 & 1.96 & 0.05 & 8.39 & 2.46 \\
\hline Atrazine & $\mathrm{H}$ & 134,669 & 167,752 & 0.21 & 0.29 & 0.05 & 7.91 & 0.48 \\
\hline
\end{tabular}


Table 5. Ranking of pesticides used in the National W ater-Quality Assessment Program study units during 1992-1995- Continued

\begin{tabular}{|c|c|c|c|c|c|c|c|c|}
\hline Compound & $\begin{array}{c}\text { Pesticide } \\
\text { type }\end{array}$ & $\begin{array}{l}\text { Average } \\
\text { amount } \\
\text { applied } \\
\text { (lb a.i.) }\end{array}$ & $\begin{array}{l}\text { Study unit } \\
\text { area treated } \\
\quad \text { (acres) }\end{array}$ & $\begin{array}{l}\text { Percentage of } \\
\text { national use }\end{array}$ & $\begin{array}{l}\text { Percentage of } \\
\text { U.S. area } \\
\text { treated }\end{array}$ & $\begin{array}{c}\text { Average } \\
\text { amount } \\
\text { applied to } \\
\text { cropland and } \\
\text { pasture area } \\
\text { (lb a.i.) }\end{array}$ & $\begin{array}{l}\text { Percentage of } \\
\text { all reported } \\
\text { pesticides } \\
\text { used in the } \\
\text { study unit }\end{array}$ & $\begin{array}{c}\text { Percentage of } \\
\text { total use } \\
\text { among study } \\
\text { units }\end{array}$ \\
\hline \multicolumn{9}{|c|}{ Trinity River Basin (TRIN) —Continued } \\
\hline Molinate & $\mathrm{H}$ & 90,350 & 27,462 & 1.84 & 1.93 & 0.03 & 5.31 & 2.22 \\
\hline Metolachlor & $\mathrm{H}$ & 85,106 & 66,917 & 0.15 & 0.21 & 0.03 & 5 & 0.31 \\
\hline Carbaryl & I & 79,292 & 59,802 & 1.74 & 2.03 & 0.03 & 4.66 & 3.4 \\
\hline Thiobencarb & $\mathrm{H}$ & 67,831 & 23,800 & 4.72 & 5.03 & 0.03 & 3.98 & 7.29 \\
\hline Trifluralin & $\mathrm{H}$ & 57,422 & 79,018 & 0.29 & 0.31 & 0.02 & 3.37 & 0.59 \\
\hline Picloram & $\mathrm{H}$ & 42,152 & 120,483 & 2.45 & 1.7 & 0.02 & 2.48 & 12.04 \\
\hline Chlorpyrifos & $\mathrm{I}$ & 41,077 & 55,928 & 0.27 & 0.4 & 0.02 & 2.41 & 0.51 \\
\hline $1,3-\mathrm{D}$ & $\mathrm{OP}$ & 39,596 & 806 & 0.1 & 0.16 & 0.01 & 2.33 & 0.16 \\
\hline Methyl parathion & I & 37,426 & 52,437 & 0.42 & 0.7 & 0.01 & 2.2 & 0.68 \\
\hline Sulfur & $\mathrm{F}$ & 34,364 & 2,042 & 0.04 & 0.08 & 0.01 & 2.02 & 0.06 \\
\hline Alachlor & $\mathrm{H}$ & 34,169 & 26,506 & 0.13 & 0.18 & 0.01 & 2.01 & 0.3 \\
\hline Terbufos & I & 24,898 & 29,757 & 0.35 & 0.45 & 0.01 & 1.46 & 0.81 \\
\hline Pendimethalin & $\mathrm{H}$ & 22,469 & 32,740 & 0.11 & 0.15 & 0.01 & 1.32 & 0.21 \\
\hline Chlorothalonil & $\mathrm{F}$ & 19,815 & 9,935 & 0.16 & 0.32 & 0.01 & 1.16 & 0.22 \\
\hline Oil & I & 19,741 & 1,354 & 0.04 & 0.1 & 0.01 & 1.16 & 0.05 \\
\hline Malathion & I & 18,800 & 8,986 & 0.61 & 0.46 & 0.01 & 1.1 & 1.31 \\
\hline Dimethoate & I & 18,106 & 41,979 & 0.53 & 0.81 & 0.01 & 1.06 & 1.64 \\
\hline Glyphosate & $\mathrm{H}$ & 17,134 & 22,640 & 0.11 & 0.1 & 0.01 & 1.01 & 0.21 \\
\hline \multicolumn{9}{|c|}{ Upper Colorado Basin (UCOL) } \\
\hline \multicolumn{9}{|c|}{ [Study Unit Area: 11,445,479 acres; Area in Cropland and Pasture: 402,027 acres] } \\
\hline $2,4-\mathrm{D}$ & $\mathrm{H}$ & 141,884 & 285,960 & 0.38 & 0.37 & 0.35 & 30.89 & 1.06 \\
\hline Sulfuric acid & OP & 95,169 & 279 & 0.37 & 0.26 & 0.24 & 20.72 & 0.44 \\
\hline EPTC & $\mathrm{H}$ & 24,535 & 7,359 & 0.17 & 0.18 & 0.06 & 5.34 & 0.37 \\
\hline Alachlor & $\mathrm{H}$ & 16,793 & 6,983 & 0.07 & 0.05 & 0.04 & 3.66 & 0.15 \\
\hline Terbufos & I & 11,899 & 9,155 & 0.17 & 0.14 & 0.03 & 2.59 & 0.39 \\
\hline Metam sodium & $\mathrm{OP}$ & 11,373 & 66 & 0.04 & 0.02 & 0.03 & 2.48 & 0.05 \\
\hline Atrazine & $\mathrm{H}$ & 10,831 & 10,039 & 0.02 & 0.02 & 0.03 & 2.36 & 0.04 \\
\hline Chlorpyrifos & I & 9,159 & 13,108 & 0.06 & 0.09 & 0.02 & 1.99 & 0.11 \\
\hline Carbofuran & I & 8,756 & 19,143 & 0.17 & 0.29 & 0.02 & 1.91 & 0.39 \\
\hline Sulfur & $\mathrm{F}$ & 8,754 & 1,893 & 0.01 & 0.08 & 0.02 & 1.91 & 0.01 \\
\hline DCPA & $\mathrm{H}$ & 8,559 & 1,247 & 0.86 & 0.68 & 0.02 & 1.86 & 2.48 \\
\hline Copper & $\mathrm{F}$ & 8,202 & 5,759 & 0.08 & 0.23 & 0.02 & 1.79 & 0.09 \\
\hline Azinphos methyl & I & 7,911 & 5,405 & 0.32 & 0.21 & 0.02 & 1.72 & 0.57 \\
\hline Metolachlor & $\mathrm{H}$ & 7,265 & 4,121 & 0.01 & 0.01 & 0.02 & 1.58 & 0.03 \\
\hline Endosulfan & I & 6,120 & 3,548 & 0.29 & 0.22 & 0.02 & 1.33 & 0.45 \\
\hline Chlorothalonil & $\mathrm{F}$ & 5,019 & 3,288 & 0.04 & 0.11 & 0.01 & 1.09 & 0.06 \\
\hline Dicamba & $\mathrm{H}$ & 4,942 & 11,431 & 0.05 & 0.03 & 0.01 & 1.08 & 0.12 \\
\hline \multicolumn{9}{|c|}{ Upper Illinois River Basin (UIRB) } \\
\hline \multicolumn{9}{|c|}{ [Study Unit Area: 6,996,354 acres; Area in Cropland and Pasture: 3,915,252 acres] } \\
\hline Atrazine & $\mathrm{H}$ & $1,855,991$ & $1,571,630$ & 2.9 & 2.76 & 0.47 & 19.44 & 6.61 \\
\hline Metolachlor & $\mathrm{H}$ & $1,500,833$ & 748,450 & 2.59 & 2.39 & 0.38 & 15.72 & 5.51 \\
\hline
\end{tabular}


Table 5. Ranking of pesticides used in the National W ater-Quality Assessment Program study units during 1992-1995- Continued

\begin{tabular}{|c|c|c|c|c|c|c|c|c|}
\hline Compound & $\begin{array}{c}\text { Pesticide } \\
\text { type }\end{array}$ & $\begin{array}{l}\text { Average } \\
\text { amount } \\
\text { applied } \\
\text { (lb a.i.) }\end{array}$ & $\begin{array}{l}\text { Study unit } \\
\text { area treated } \\
\text { (acres) }\end{array}$ & $\begin{array}{l}\text { Percentage of } \\
\text { national use }\end{array}$ & $\begin{array}{c}\text { Percentage of } \\
\text { U.S. area } \\
\text { treated }\end{array}$ & $\begin{array}{c}\text { Average } \\
\text { amount } \\
\text { applied to } \\
\text { cropland and } \\
\text { pasture area } \\
\text { (lb a.i.) }\end{array}$ & $\begin{array}{l}\text { Percentage of } \\
\text { all reported } \\
\text { pesticides } \\
\text { used in the } \\
\text { study unit }\end{array}$ & $\begin{array}{c}\text { Percentage of } \\
\text { total use } \\
\text { among study } \\
\text { units }\end{array}$ \\
\hline \multicolumn{9}{|c|}{ Upper Illinois River Basin (UIRB) —Continued } \\
\hline Cyanazine & $\mathrm{H}$ & $1,118,412$ & 467,558 & 3.79 & 2.95 & 0.29 & 11.72 & 8.07 \\
\hline Acetochlor & $\mathrm{H}$ & 958,521 & 454,666 & 4.02 & 3.84 & 0.24 & 10.04 & 8.65 \\
\hline Pendimethalin & $\mathrm{H}$ & 606,779 & 620,315 & 2.93 & 2.89 & 0.15 & 6.36 & 5.66 \\
\hline Alachlor & $\mathrm{H}$ & 568,865 & 275,503 & 2.22 & 1.9 & 0.15 & 5.96 & 4.98 \\
\hline Glyphosate & $\mathrm{H}$ & 283,344 & 487,709 & 1.77 & 2.05 & 0.07 & 2.97 & 3.41 \\
\hline EPTC & $\mathrm{H}$ & 245,111 & 57,511 & 1.74 & 1.43 & 0.06 & 2.57 & 3.72 \\
\hline $2,4-\mathrm{D}$ & $\mathrm{H}$ & 231,639 & 571,423 & 0.62 & 0.74 & 0.06 & 2.43 & 1.73 \\
\hline Butylate & $\mathrm{H}$ & 223,925 & 59,122 & 2.91 & 2.98 & 0.06 & 2.35 & 7.52 \\
\hline Dicamba & $\mathrm{H}$ & 184,480 & 452,016 & 1.92 & 1.16 & 0.05 & 1.93 & 4.54 \\
\hline Chlorpyrifos & I & 165,139 & 160,003 & 1.07 & 1.15 & 0.04 & 1.73 & 2.05 \\
\hline Trifluralin & $\mathrm{H}$ & 160,145 & 204,481 & 0.81 & 0.81 & 0.04 & 1.68 & 1.63 \\
\hline Terbufos & I & 158,292 & 136,131 & 2.2 & 2.04 & 0.04 & 1.66 & 5.13 \\
\hline Bentazon & $\mathrm{H}$ & 154,316 & 241,563 & 2.29 & 2.41 & 0.04 & 1.62 & 3.97 \\
\hline Simazine & $\mathrm{H}$ & 134,897 & 104,967 & 2.8 & 3.06 & 0.03 & 1.41 & 4.4 \\
\hline \multicolumn{9}{|c|}{ Upper Mississippi River Basin (UMIS) } \\
\hline \multicolumn{9}{|c|}{ [Study Unit Area: 30,291,534 acres; Area in Cropland and Pasture: 12,053,794 acres] } \\
\hline Acetochlor & $\mathrm{H}$ & $2,469,593$ & $1,281,828$ & 10.36 & 10.82 & 0.2 & 13.4 & 22.28 \\
\hline Metolachlor & $\mathrm{H}$ & $2,397,267$ & $1,135,083$ & 4.14 & 3.63 & 0.2 & 13 & 8.81 \\
\hline Atrazine & $\mathrm{H}$ & $1,538,189$ & $1,951,098$ & 2.41 & 3.42 & 0.13 & 8.34 & 5.48 \\
\hline EPTC & $\mathrm{H}$ & $1,295,937$ & 309,867 & 9.19 & 7.72 & 0.11 & 7.03 & 19.65 \\
\hline Pendimethalin & $\mathrm{H}$ & $1,172,831$ & 960,108 & 5.66 & 4.47 & 0.1 & 6.36 & 10.93 \\
\hline Alachlor & $\mathrm{H}$ & $1,161,163$ & 551,096 & 4.53 & 3.79 & 0.1 & 6.3 & 10.17 \\
\hline Cyanazine & $\mathrm{H}$ & $1,128,232$ & 693,975 & 3.82 & 4.38 & 0.09 & 6.12 & 8.14 \\
\hline Trifluralin & $\mathrm{H}$ & $1,078,748$ & $1,408,420$ & 5.45 & 5.55 & 0.09 & 5.85 & 11 \\
\hline Dicamba & $\mathrm{H}$ & 978,390 & $2,907,019$ & 10.2 & 7.46 & 0.08 & 5.31 & 24.06 \\
\hline $2,4-\mathrm{D}$ & $\mathrm{H}$ & 542,984 & $1,147,354$ & 1.46 & 1.48 & 0.05 & 2.95 & 4.04 \\
\hline Dimethenamid & $\mathrm{H}$ & 532,884 & 483,818 & 20.37 & 20.84 & 0.04 & 2.89 & 40.64 \\
\hline Bentazon & $\mathrm{H}$ & 495,004 & 695,371 & 7.36 & 6.94 & 0.04 & 2.69 & 12.75 \\
\hline Glyphosate & $\mathrm{H}$ & 368,972 & 547,939 & 2.3 & 2.3 & 0.03 & 2 & 4.44 \\
\hline Propachlor & $\mathrm{H}$ & 365,805 & 151,494 & 9.46 & 9.05 & 0.03 & 1.98 & 27.79 \\
\hline Terbufos & I & 237,239 & 210,992 & 3.3 & 3.16 & 0.02 & 1.29 & 7.69 \\
\hline Chlorpyrifos & I & 223,869 & 217,170 & 1.45 & 1.56 & 0.02 & 1.21 & 2.78 \\
\hline \multicolumn{9}{|c|}{ Upper Snake River Basin (USNK) } \\
\hline \multicolumn{9}{|c|}{ [Study Unit Area: $22,931,020$ acres; Area in Cropland and Pasture: $2,017,8868$ acres] } \\
\hline Sulfuric acid & $\mathrm{OP}$ & $15,759,582$ & 55,078 & 60.75 & 52.15 & 5.59 & 45.38 & 72.54 \\
\hline Metam sodium & OP & $8,005,645$ & 51,636 & 24.83 & 13.45 & 2.84 & 23.05 & 32.66 \\
\hline $1,3-\mathrm{D}$ & OP & $5,493,173$ & 33,601 & 13.73 & 6.82 & 1.95 & 15.82 & 22.36 \\
\hline Sulfur & $\mathrm{F}$ & $1,208,681$ & 61,713 & 1.39 & 2.5 & 0.43 & 3.48 & 1.99 \\
\hline EPTC & $\mathrm{H}$ & 739,252 & 233,544 & 5.24 & 5.82 & 0.26 & 2.13 & 11.21 \\
\hline Phorate & I & 440,639 & 163,228 & 11.56 & 5.43 & 0.16 & 1.27 & 20.14 \\
\hline
\end{tabular}


Table 5. Ranking of pesticides used in the National W ater-Quality Assessment Program study units during 1992-1995- Continued

\begin{tabular}{|c|c|c|c|c|c|c|c|c|}
\hline Compound & $\begin{array}{c}\text { Pesticide } \\
\text { type }\end{array}$ & $\begin{array}{c}\text { Average } \\
\text { amount } \\
\text { applied } \\
\text { (lb a.i.) }\end{array}$ & $\begin{array}{l}\text { Study unit } \\
\text { area treated } \\
\text { (acres) }\end{array}$ & $\begin{array}{l}\text { Percentage of } \\
\text { national use }\end{array}$ & $\begin{array}{l}\text { Percentage of } \\
\text { U.S. area } \\
\text { treated }\end{array}$ & $\begin{array}{c}\text { Average } \\
\text { amount } \\
\text { applied to } \\
\text { cropland and } \\
\text { pasture area } \\
\text { (lb a.i.) }\end{array}$ & $\begin{array}{l}\text { Percentage of } \\
\text { all reported } \\
\text { pesticides } \\
\text { used in the } \\
\text { study unit }\end{array}$ & $\begin{array}{c}\text { Percentage of } \\
\text { total use } \\
\text { among study } \\
\text { units }\end{array}$ \\
\hline \multicolumn{9}{|c|}{ Upper Tennessee River Basin (UTEN) } \\
\hline \multicolumn{9}{|c|}{ [Study Unit Area: $13,690,767$ acres; Area in Cropland and Pasture: $1,868,185$ acres] } \\
\hline Methyl bromide & $\mathrm{OP}$ & 422,986 & 1,124 & 1.08 & 0.55 & 0.23 & 16.72 & 1.51 \\
\hline $1,3-\mathrm{D}$ & $\mathrm{OP}$ & 341,932 & 4,908 & 0.85 & 1 & 0.18 & 13.52 & 1.39 \\
\hline Oil & I & 256,370 & 7,097 & 0.48 & 0.52 & 0.14 & 10.14 & 0.66 \\
\hline $2,4-\mathrm{D}$ & $\mathrm{H}$ & 139,182 & 246,941 & 0.37 & 0.32 & 0.07 & 5.5 & 1.04 \\
\hline Maleic hydrazide & $\mathrm{OP}$ & 132,968 & 42,917 & 6.26 & 5.95 & 0.07 & 5.26 & 12.31 \\
\hline Atrazine & $\mathrm{H}$ & 116,252 & 83,304 & 0.18 & 0.15 & 0.06 & 4.6 & 0.41 \\
\hline Captan & $\mathrm{F}$ & 108,361 & 7,567 & 2.85 & 1.29 & 0.06 & 4.28 & 5.56 \\
\hline Acephate & I & 80,714 & 55,896 & 2.22 & 1.75 & 0.04 & 3.19 & 3.91 \\
\hline Chlorpyrifos & I & 71,534 & 38,161 & 0.46 & 0.27 & 0.04 & 2.83 & 0.89 \\
\hline Ziram & $\mathrm{F}$ & 69,549 & 3,719 & 2.52 & 0.76 & 0.04 & 2.75 & 3.22 \\
\hline Sulfur & $\mathrm{F}$ & 58,651 & 2,151 & 0.07 & 0.09 & 0.03 & 2.32 & 0.1 \\
\hline Metolachlor & $\mathrm{H}$ & 46,256 & 29,725 & 0.08 & 0.1 & 0.02 & 1.83 & 0.17 \\
\hline Chloropicrin & $\mathrm{OP}$ & 45,086 & 916 & 0.61 & 0.75 & 0.02 & 1.78 & 1.05 \\
\hline Alachlor & $\mathrm{H}$ & 40,879 & 21,628 & 0.16 & 0.15 & 0.02 & 1.62 & 0.36 \\
\hline Mancozeb & $\mathrm{F}$ & 40,445 & 5,745 & 0.49 & 0.28 & 0.02 & 1.6 & 0.75 \\
\hline Carbaryl & I & 38,473 & 22,377 & 0.85 & 0.76 & 0.02 & 1.52 & 1.65 \\
\hline Pebulate & $\mathrm{H}$ & 31,366 & 7,831 & 5.6 & 4.98 & 0.02 & 1.24 & 9.72 \\
\hline Metalaxyl & $\mathrm{F}$ & 28,128 & 56,333 & 3.17 & 2.3 & 0.02 & 1.11 & 5.4 \\
\hline \multicolumn{9}{|c|}{ White River Basin (WHIT) } \\
\hline \multicolumn{9}{|c|}{ [Study Unit Area: 7,260,658 acres; Area in Cropland and Pasture: 3,492,449 acres] } \\
\hline Atrazine & $\mathrm{H}$ & $1,904,800$ & $1,410,990$ & 2.98 & 2.48 & 0.55 & 24.59 & 6.78 \\
\hline Metolachlor & $\mathrm{H}$ & $1,177,058$ & 618,727 & 2.03 & 1.98 & 0.34 & 15.2 & 4.32 \\
\hline Acetochlor & $\mathrm{H}$ & 789,630 & 340,358 & 3.31 & 2.87 & 0.23 & 10.2 & 7.13 \\
\hline Cyanazine & $\mathrm{H}$ & 678,351 & 308,395 & 2.3 & 1.95 & 0.19 & 8.76 & 4.9 \\
\hline Alachlor & $\mathrm{H}$ & 537,903 & 275,544 & 2.1 & 1.9 & 0.15 & 6.95 & 4.71 \\
\hline Pendimethalin & $\mathrm{H}$ & 370,900 & 418,837 & 1.79 & 1.95 & 0.11 & 4.79 & 3.46 \\
\hline Glyphosate & $\mathrm{H}$ & 343,055 & 608,844 & 2.14 & 2.56 & 0.1 & 4.43 & 4.13 \\
\hline Butylate & $\mathrm{H}$ & 341,979 & 81,038 & 4.44 & 4.08 & 0.1 & 4.42 & 11.49 \\
\hline $2,4-\mathrm{D}$ & $\mathrm{H}$ & 224,257 & 538,633 & 0.6 & 0.69 & 0.06 & 2.9 & 1.67 \\
\hline EPTC & $\mathrm{H}$ & 133,877 & 32,416 & 0.95 & 0.81 & 0.04 & 1.73 & 2.03 \\
\hline Bentazon & $\mathrm{H}$ & 127,340 & 186,847 & 1.89 & 1.87 & 0.04 & 1.64 & 3.28 \\
\hline Dicamba & $\mathrm{H}$ & 97,294 & 255,935 & 1.02 & 0.66 & 0.03 & 1.26 & 2.39 \\
\hline Terbufos & I & 90,940 & 81,215 & 1.26 & 1.22 & 0.03 & 1.17 & 2.95 \\
\hline Trifluralin & $\mathrm{H}$ & 83,328 & 91,824 & 0.42 & 0.36 & 0.02 & 1.08 & 0.85 \\
\hline \multicolumn{9}{|c|}{ Willamette Basin (WILL) } \\
\hline \multicolumn{9}{|c|}{ [Study Unit Area: 7,660,515 acres; Area in Cropland and Pasture: $1,014,018$ acres] } \\
\hline Diuron & $\mathrm{H}$ & 392,901 & 313,386 & 10.49 & 9.65 & 0.39 & 12.76 & 14.52 \\
\hline Sulfur & $\mathrm{F}$ & 325,640 & 43,883 & 0.38 & 1.78 & 0.32 & 10.57 & 0.54 \\
\hline Oil & I & 218,819 & 8,527 & 0.41 & 0.63 & 0.22 & 7.11 & 0.57 \\
\hline
\end{tabular}


Table 5. Ranking of pesticides used in the National W ater-Quality Assessment Program study units during 1992-1995- Continued

\begin{tabular}{|c|c|c|c|c|c|c|c|c|}
\hline Compound & $\begin{array}{c}\text { Pesticide } \\
\text { type }\end{array}$ & $\begin{array}{l}\text { Average } \\
\text { amount } \\
\text { applied } \\
\text { (lb a.i.) }\end{array}$ & $\begin{array}{l}\text { Study unit } \\
\text { area treated } \\
\text { (acres) }\end{array}$ & $\begin{array}{l}\text { Percentage of } \\
\text { national use }\end{array}$ & $\begin{array}{c}\text { Percentage of } \\
\text { U.S. area } \\
\text { treated }\end{array}$ & $\begin{array}{c}\text { Average } \\
\text { amount } \\
\text { applied to } \\
\text { cropland and } \\
\text { pasture area } \\
\text { (lb a.i.) } \\
\end{array}$ & $\begin{array}{l}\text { Percentage of } \\
\text { all reported } \\
\text { pesticides } \\
\text { used in the } \\
\text { study unit }\end{array}$ & $\begin{array}{c}\text { Percentage of } \\
\text { total use } \\
\text { among study } \\
\text { units }\end{array}$ \\
\hline \multicolumn{9}{|c|}{ Willamette Basin (WILL) —Continued } \\
\hline $1,3-\mathrm{D}$ & $\mathrm{OP}$ & 171,158 & 1,084 & 0.43 & 0.22 & 0.17 & 5.56 & 0.7 \\
\hline $2,4-\mathrm{D}$ & $\mathrm{H}$ & 151,273 & 275,839 & 0.41 & 0.36 & 0.15 & 4.91 & 1.13 \\
\hline EPTC & $\mathrm{H}$ & 117,138 & 36,853 & 0.83 & 0.92 & 0.12 & 3.8 & 1.78 \\
\hline Glyphosate & $\mathrm{H}$ & 108,054 & 156,572 & 0.67 & 0.66 & 0.11 & 3.51 & 1.3 \\
\hline Copper & $\mathrm{F}$ & 94,504 & 23,128 & 0.9 & 0.92 & 0.09 & 3.07 & 1.09 \\
\hline Chlorpyrifos & I & 88,635 & 75,138 & 0.58 & 0.54 & 0.09 & 2.88 & 1.1 \\
\hline Metolachlor & $\mathrm{H}$ & 81,493 & 39,632 & 0.14 & 0.13 & 0.08 & 2.65 & 0.3 \\
\hline MCPA & $\mathrm{H}$ & 69,280 & 148,609 & 1.49 & 1.21 & 0.07 & 2.25 & 3.07 \\
\hline Chlorothalonil & $\mathrm{F}$ & 54,300 & 36,043 & 0.42 & 1.15 & 0.05 & 1.76 & 0.6 \\
\hline Atrazine & $\mathrm{H}$ & 51,152 & 38,288 & 0.08 & 0.07 & 0.05 & 1.66 & 0.18 \\
\hline Propargite & I & 48,712 & 27,381 & 1.42 & 1.41 & 0.05 & 1.58 & 2.26 \\
\hline Paraquat & $\mathrm{H}$ & 44,676 & 69,036 & 1.06 & 0.73 & 0.04 & 1.45 & 1.9 \\
\hline Propiconazole & $\mathrm{F}$ & 44,412 & 314,770 & 15.24 & 17.07 & 0.04 & 1.44 & 23.59 \\
\hline Bentazon & $\mathrm{H}$ & 44,177 & 31,908 & 0.66 & 0.32 & 0.04 & 1.43 & 1.14 \\
\hline Fonofos & I & 40,246 & 22,034 & 1.43 & 0.91 & 0.04 & 1.31 & 3.18 \\
\hline Dicamba & $\mathrm{H}$ & 39,338 & 285,589 & 0.41 & 0.73 & 0.04 & 1.28 & 0.97 \\
\hline Carbaryl & I & 38,863 & 21,783 & 0.85 & 0.74 & 0.04 & 1.26 & 1.67 \\
\hline Ethofumesate & $\mathrm{H}$ & 37,467 & 107,049 & 9.74 & 24.02 & 0.04 & 1.22 & 26.65 \\
\hline Ethoprop & I & 33,114 & 18,235 & 2.61 & 5.42 & 0.03 & 1.08 & 3.72 \\
\hline Simazine & $\mathrm{H}$ & 32,784 & 22,630 & 0.68 & 0.66 & 0.03 & 1.06 & 1.07 \\
\hline Alachlor & $\mathrm{H}$ & 31,337 & 11,623 & 0.12 & 0.08 & 0.03 & 1.02 & 0.27 \\
\hline Diazinon & I & 30,911 & 22,022 & 1.91 & 2.05 & 0.03 & 1 & 2.63 \\
\hline \multicolumn{9}{|c|}{ Western Lake Michigan Drainage (WMIC) } \\
\hline \multicolumn{9}{|c|}{ [Study Unit Area: 12,780,389 acres; Area in Cropland and Pasture: 2,817,763 acres] } \\
\hline Metolachlor & $\mathrm{H}$ & 592,116 & 312,436 & 1.02 & 1 & 0.21 & 12.01 & 2.18 \\
\hline Cyanazine & $\mathrm{H}$ & 504,440 & 305,293 & 1.71 & 1.93 & 0.18 & 10.23 & 3.64 \\
\hline Sulfuric acid & $\mathrm{OP}$ & 488,998 & 3,252 & 1.89 & 3.08 & 0.17 & 9.92 & 2.25 \\
\hline Atrazine & $\mathrm{H}$ & 484,322 & 572,840 & 0.76 & 1.01 & 0.17 & 9.82 & 1.73 \\
\hline Metam sodium & $\mathrm{OP}$ & 325,558 & 2,216 & 1.01 & 0.58 & 0.12 & 6.6 & 1.33 \\
\hline Alachlor & $\mathrm{H}$ & 274,719 & 149,822 & 1.07 & 1.03 & 0.1 & 5.57 & 2.41 \\
\hline EPTC & $\mathrm{H}$ & 252,897 & 72,744 & 1.79 & 1.81 & 0.09 & 5.13 & 3.84 \\
\hline Mancozeb & $\mathrm{F}$ & 204,486 & 33,639 & 2.46 & 1.65 & 0.07 & 4.15 & 3.81 \\
\hline Dicamba & $\mathrm{H}$ & 182,659 & 437,033 & 1.91 & 1.12 & 0.06 & 3.7 & 4.49 \\
\hline Pendimethalin & $\mathrm{H}$ & 178,916 & 141,664 & 0.86 & 0.66 & 0.06 & 3.63 & 1.67 \\
\hline Chlorothalonil & $\mathrm{F}$ & 151,837 & 29,670 & 1.19 & 0.95 & 0.05 & 3.08 & 1.68 \\
\hline Dimethenamid & $\mathrm{H}$ & 115,600 & 91,746 & 4.42 & 3.95 & 0.04 & 2.34 & 8.82 \\
\hline Chlorpyrifos & I & 115,240 & 117,838 & 0.75 & 0.84 & 0.04 & 2.34 & 1.43 \\
\hline Glyphosate & $\mathrm{H}$ & 94,692 & 107,619 & 0.59 & 0.45 & 0.03 & 1.92 & 1.14 \\
\hline Oil & I & 90,429 & 2,322 & 0.17 & 0.17 & 0.03 & 1.83 & 0.23 \\
\hline Terbufos & I & 78,566 & 74,554 & 1.09 & 1.12 & 0.03 & 1.59 & 2.55 \\
\hline
\end{tabular}


Table 5. Ranking of pesticides used in the National W ater-Quality Assessment Program study units during 1992-1995- Continued

\begin{tabular}{|c|c|c|c|c|c|c|c|c|}
\hline Compound & $\begin{array}{c}\text { Pesticide } \\
\text { type }\end{array}$ & $\begin{array}{l}\text { Average } \\
\text { amount } \\
\text { applied } \\
\text { (lb a.i.) }\end{array}$ & $\begin{array}{l}\text { Study unit } \\
\text { area treated } \\
\text { (acres) }\end{array}$ & $\begin{array}{l}\text { Percentage of } \\
\text { national use }\end{array}$ & $\begin{array}{l}\text { Percentage of } \\
\text { U.S. area } \\
\text { treated }\end{array}$ & $\begin{array}{c}\text { Average } \\
\text { amount } \\
\text { applied to } \\
\text { cropland and } \\
\text { pasture area } \\
\text { (lb a.i.) }\end{array}$ & $\begin{array}{l}\text { Percentage of } \\
\text { all reported } \\
\text { pesticides } \\
\text { used in the } \\
\text { study unit }\end{array}$ & $\begin{array}{c}\text { Percentage of } \\
\text { total use } \\
\text { among study } \\
\text { units }\end{array}$ \\
\hline \multicolumn{9}{|c|}{ Western Lake Michigan Drainage (WMIC) —Continued } \\
\hline Maneb & $\mathrm{F}$ & 60,370 & 12,491 & 2.01 & 1.78 & 0.02 & 1.22 & 3.51 \\
\hline 2,4-D & $\mathrm{H}$ & 53,683 & 108,670 & 0.14 & 0.14 & 0.02 & 1.09 & 0.4 \\
\hline \multicolumn{9}{|c|}{ Yakima River Basin (YAKI) } \\
\hline \multicolumn{9}{|c|}{ [Study Unit Area: 3,931,978 acres; Area in Cropland and Pasture: 422,213 acres] } \\
\hline Oil & I & $2,392,999$ & 62,744 & 4.5 & 4.63 & 5.67 & 42.03 & 6.2 \\
\hline Metam sodium & OP & $1,005,122$ & 6,506 & 3.12 & 1.7 & 2.38 & 17.65 & 4.1 \\
\hline $1,3-\mathrm{D}$ & $\mathrm{OP}$ & 396,975 & 2,448 & 0.99 & 0.5 & 0.94 & 6.97 & 1.62 \\
\hline Sulfur & $\mathrm{F}$ & 203,203 & 24,779 & 0.23 & 1 & 0.48 & 3.57 & 0.33 \\
\hline Sulfuric acid & $\mathrm{OP}$ & 191,371 & 932 & 0.74 & 0.88 & 0.45 & 3.36 & 0.88 \\
\hline Azinphos methyl & $\mathrm{I}$ & 104,671 & 54,799 & 4.2 & 2.12 & 0.25 & 1.84 & 7.48 \\
\hline Chlorpyrifos & I & 96,895 & 49,650 & 0.63 & 0.36 & 0.23 & 1.7 & 1.2 \\
\hline $2,4-\mathrm{D}$ & $\mathrm{H}$ & 96,009 & 175,097 & 0.26 & 0.23 & 0.23 & 1.69 & 0.72 \\
\hline Mancozeb & $\mathrm{F}$ & 77,636 & 19,954 & 0.94 & 0.98 & 0.18 & 1.36 & 1.45 \\
\hline Ziram & $\mathrm{F}$ & 73,975 & 15,983 & 2.68 & 3.24 & 0.18 & 1.3 & 3.43 \\
\hline Carbaryl & I & 57,247 & 39,938 & 1.26 & 1.35 & 0.14 & 1.01 & 2.45 \\
\hline \multicolumn{9}{|c|}{ Yellowstone River Basin (YELL) } \\
\hline \multicolumn{9}{|c|}{ [Study Unit Area: 44,868,888 acres: Area in Cropland and Pasture: $2,556,753$ acres] } \\
\hline $1,3-\mathrm{D}$ & $\mathrm{OP}$ & $1,158,519$ & 8,032 & 2.9 & 1.63 & 0.45 & 44.34 & 4.72 \\
\hline 2,4-D & $\mathrm{H}$ & 468,317 & $1,158,219$ & 1.26 & 1.49 & 0.18 & 17.93 & 3.49 \\
\hline Triallate & $\mathrm{H}$ & 89,929 & 90,848 & 3.94 & 4.08 & 0.04 & 3.44 & 8.98 \\
\hline Aldicarb & I & 69,466 & 27,907 & 1.62 & 0.65 & 0.03 & 2.66 & 2.19 \\
\hline Cycloate & $\mathrm{H}$ & 63,650 & 36,543 & 6.87 & 10.05 & 0.02 & 2.44 & 10.75 \\
\hline Dicamba & $\mathrm{H}$ & 62,607 & 650,181 & 0.65 & 1.67 & 0.02 & 2.4 & 1.54 \\
\hline Glyphosate & $\mathrm{H}$ & 62,009 & 46,294 & 0.39 & 0.19 & 0.02 & 2.37 & 0.75 \\
\hline Terbufos & I & 59,633 & 56,752 & 0.83 & 0.85 & 0.02 & 2.28 & 1.93 \\
\hline Picloram & $\mathrm{H}$ & 59,009 & 246,809 & 3.42 & 3.47 & 0.02 & 2.26 & 16.85 \\
\hline MCPA & $\mathrm{H}$ & 46,954 & 135,807 & 1.01 & 1.1 & 0.02 & 1.8 & 2.08 \\
\hline EPTC & $\mathrm{H}$ & 43,157 & 14,449 & 0.31 & 0.36 & 0.02 & 1.65 & 0.65 \\
\hline Ethofumesate & $\mathrm{H}$ & 39,063 & 44,540 & 10.16 & 10 & 0.02 & 1.5 & 27.78 \\
\hline Diethatyl ethyl & $\mathrm{H}$ & 36,006 & 31,757 & 9.06 & 11.88 & 0.01 & 1.38 & 24.82 \\
\hline Diclofop & $\mathrm{H}$ & 35,384 & 52,714 & 3.17 & 3.75 & 0.01 & 1.35 & 5.71 \\
\hline Malathion & I & 27,342 & 23,281 & 0.88 & 1.2 & 0.01 & 1.05 & 1.9 \\
\hline
\end{tabular}

